\title{
Uniform Bounds for the Bilinear Hilbert Transforms, II
}

Xiaochun Li

\begin{abstract}
We continue the investigation initiated in [8] of uniform $L^{p}$ bounds for the family of bilinear Hilbert transforms

$$
H_{\alpha, \beta}(f, g)(x)=\text { p.v. } \int_{\mathbb{R}} f(x-\alpha t) g(x-\beta t) \frac{d t}{t} .
$$

In this work we show that $H_{\alpha, \beta}$ map $L^{p_{1}}(\mathbb{R}) \times L^{p_{2}}(\mathbb{R})$ into $L^{p}(\mathbb{R})$ uniformly in the real parameters $\alpha, \beta$ satisfying $\left|\frac{\alpha}{\beta}-1\right| \geq c>0$ when $1<p_{1}, p_{2}<2$ and $\frac{2}{3}<p=\frac{p_{1} p_{2}}{p_{1}+p_{2}}<\infty$. As a corollary we obtain $L^{p} \times L^{\infty} \rightarrow L^{p}$ uniform bounds in the range $4 / 3<p<4$ for the $H_{1, \alpha}$ 's when $\alpha \in[0,1)$.
\end{abstract}

\section{Introduction}

The family of bilinear Hilbert transforms was introduced by A. Calderón in one of his early attempts to derive boundedness for the Cauchy integral along Lipschitz curves. The bilinear Hilbert transform in the direction $(\alpha, \beta) \in \mathbb{R}^{2}$ is defined by

$$
H_{\alpha, \beta}(f, g)(x)=\mathrm{p} . \mathrm{v} \cdot \int_{\mathbb{R}} f(x-\alpha t) g(x-\beta t) \frac{d t}{t},
$$

where $f$ and $g$ are Schwartz functions on the line. This definition can be extended to the case when one of the two parameters $\alpha$ or $\beta$ are infinity (but not both) by setting $H_{\infty, \beta}(f, g)=(H f) g$ and likewise $H_{\alpha, \infty}(f, g)=f(H g)$, where $H$ is the usual Hilbert transform on $\mathbb{R}$.

2000 Mathematics Subject Classification: Primary: 42B20, 42B25. Secondary: 46B70, 47B38.

Keywords: Time-frequency analysis, bilinear Hilbert transform, uniform bounds. 
In [11] and [12], M. Lacey and C. Thiele gave a brilliant proof of the boundedness for the operators $H_{1, \alpha}$. And later in [14] and [15], C. Thiele proved that $L^{2} \times L^{\infty} \rightarrow L^{2, \infty}$ uniform bounds for the $H_{1, \alpha}$ 's when $\alpha \in[0,1)$. Based on the ideas in $[11,12]$ and Thiele's powerful ideas in $[14,15]$, L. Grafakos and the author obtained in [8] $L^{p}$ bounds for $H_{\alpha, \beta}$ uniformly in the real parameters $\alpha, \beta$ when $2<p_{1}, p_{2}<\infty$ and $1<p<2$. In this work we continue the work in [8] to obtain $L^{p}$ bounds for $H_{\alpha, \beta}$ uniformly in $\alpha, \beta$ whose ratio stays away from a neighborhood of the number 1 when $1<p_{1}, p_{2}<2$ and $\frac{2}{3}<p<1$.

Since $H_{\alpha_{1}, \alpha_{2}}\left(f_{1}, f_{2}\right)$ reduces to $H\left(f_{1} f_{2}\right)$ when $\alpha_{1}=\alpha_{2}$, it follows that no uniform estimates can hold for $H_{\alpha_{1}, \alpha_{2}}$ from $L^{p_{1}}(\mathbb{R}) \times L^{p_{2}}(\mathbb{R})$ into $L^{p}(\mathbb{R})$ when $p \leq 1$. This restriction does not arise, however, when one seeks uniform bounds in the parameter $\alpha_{2} / \alpha_{1}$ away from a small neighborhood of the number 1. For these $\alpha_{1}, \alpha_{2}$ we are able to obtain uniform bounds when $\frac{2}{3}<p<1$. The main result of this article is the following theorem.

Theorem 1. Let $1<p_{1}, p_{2}<2$ and $\frac{2}{3}<p=\frac{p_{1} p_{2}}{p_{1}+p_{2}}<1$. Then for any $\varepsilon>0$ there is a constant $C=C\left(p_{1}, p_{2}, \varepsilon\right)$ such that for all $f_{1}, f_{2}$ Schwartz functions on $\mathbb{R}$ and all $\alpha_{1}, \alpha_{2} \in \mathbb{R}$ satisfying $\left|\frac{\alpha_{2}}{\alpha_{1}}-1\right| \geq \varepsilon$ we have

$$
\left\|H_{\alpha_{1}, \alpha_{2}}\left(f_{1}, f_{2}\right)\right\|_{p} \leq C\left\|f_{1}\right\|_{p_{1}}\left\|f_{2}\right\|_{p_{2}} .
$$

Combining this theorem with Theorem 1 in [8] we obtain that $L^{p} \times L^{\infty} \rightarrow L^{p}$ uniform bounds for $4 / 3<p<4$ for the $H_{1, \alpha}$ 's when $\alpha \in[0,1)$.

Theorem 2. Let $1<p_{1}, p_{2}<\infty$ and $1<p=\frac{p_{1} p_{2}}{p_{1}+p_{2}}<\infty$. Suppose that

$$
\left|\frac{1}{p_{1}}-\frac{1}{p_{2}}\right|<\frac{1}{2}, \quad\left|\frac{1}{p_{1}}-\frac{1}{p^{\prime}}\right|<\frac{1}{2}, \quad\left|\frac{1}{p_{2}}-\frac{1}{p^{\prime}}\right|<\frac{1}{2} .
$$

Then there is a constant $C=C\left(p_{1}, p_{2}\right)$ such that for all $f_{1}, f_{2}$ Schwartz functions on $\mathbb{R}$ we have

$$
\sup _{\alpha_{1}, \alpha_{2} \in \mathbb{R}}\left\|H_{\alpha_{1}, \alpha_{2}}\left(f_{1}, f_{2}\right)\right\|_{p} \leq C\left\|f_{1}\right\|_{p_{1}}\left\|f_{2}\right\|_{p_{2}} .
$$

Moreover, for any $\varepsilon>0$ and all $4 / 3<q<4$ there exists a constant $C=$ $C(q, \varepsilon)<\infty$ such that for all $f_{1}, f_{2}$ Schwartz functions on $\mathbb{R}$ we have

$$
\begin{gathered}
\sup _{\left|\alpha_{2} / \alpha_{1}\right| \leq \varepsilon^{-1}}\left\|H_{\alpha_{1}, \alpha_{2}}\left(f_{1}, f_{2}\right)\right\|_{q} \leq C\left\|f_{1}\right\|_{q}\left\|f_{2}\right\|_{\infty}, \\
\sup _{\left|\alpha_{2} / \alpha_{1}\right| \geq \varepsilon}\left\|H_{\alpha_{1}, \alpha_{2}}\left(f_{1}, f_{2}\right)\right\|_{q} \leq C\left\|f_{1}\right\|_{\infty}\left\|f_{2}\right\|_{q}, \\
\sup _{\left|\frac{\alpha_{2}}{\alpha_{1}}-1\right| \geq \varepsilon}\left\|H_{\alpha_{1}, \alpha_{2}}\left(f_{1}, f_{2}\right)\right\|_{1} \leq C\left\|f_{1}\right\|_{q}\left\|f_{2}\right\|_{q^{\prime}},
\end{gathered}
$$

where $q^{\prime}=q /(q-1)$. 
Furthermore, it is easy to see that (1.3), (1.4), and (1.5) fail if $\alpha_{1} / \alpha_{2}$ is unrestricted. Thus (1.3), (1.4), and (1.5) are the best possible uniform strong type endpoint estimates for the family $H_{\alpha_{1}, \alpha_{2}}$ in the range $4 / 3<q<4$. Uniform estimates for the remaining $q$ 's remain open at the moment. Also open remains the issue of whether $L^{1}$ can be replaced by weak $L^{1, \infty}$ if the restriction on $\alpha_{1}, \alpha_{2}$ is dropped in (1.5).

The boundedness of the first commutator on $L^{p}(\mathbb{R})$ is a consequence of estimate (1.3) above. For another application of these results we refer to section 9 .

We now prove Theorem 2 assuming Theorem 1.

Proof. We present the interpolation argument of the proof in a geometric fashion. Figure 1 represents the set of all $\left(1 / p_{1}, 1 / p_{2}, 1 / p\right)$ which satisfy $1 / p_{1}+1 / p_{2}=1 / p$ and $1 \leq p_{1}, p_{2} \leq \infty$.

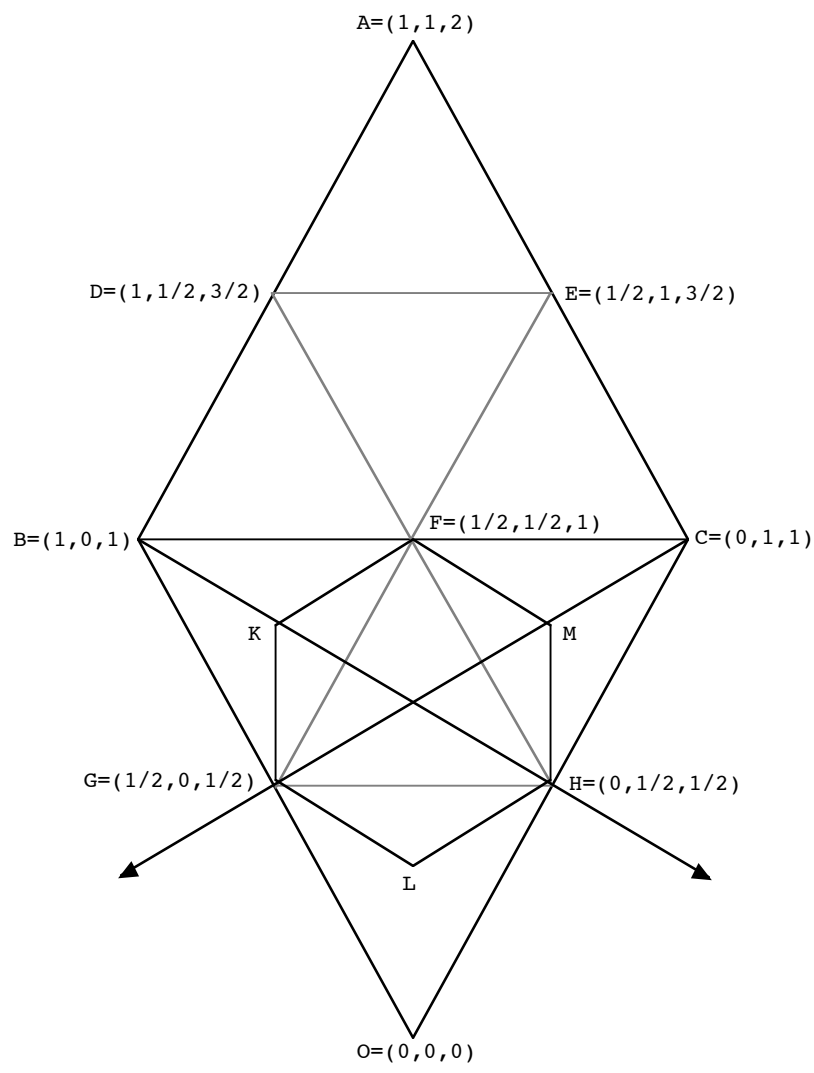

Figure 1 . The set of all $\left(1 / p_{1}, 1 / p_{2}, 1 / p\right)$ which satisfy $1 / p_{1}+1 / p_{2}=1 / p$.

First we note that the results in [11] and [12] give estimates for $H_{\alpha_{1}, \alpha_{2}}$ inside the triangle $B O C$ uniformly in $\alpha_{1}, \alpha_{2}$ which satisfy $\left|\frac{\alpha_{2}}{\alpha_{1}}-1\right| \geq c_{1}>0$ and $0<c_{1} \leq\left|\frac{\alpha_{2}}{\alpha_{1}}\right| \leq c_{2}<\infty$. Here we obtain uniform bounds near the 
bad directions in which the ratio $\frac{\alpha_{2}}{\alpha_{1}}$ approaches 0,1 , and $\infty$ in the range of exponents claimed in Theorem 2 .

We observe that the set of indices that satisfy conditions (1.1) is the hexagon $K G L H M F$. The line $C G$ is the axis of symmetry with respect to the adjoint $H_{\alpha_{1}, \alpha_{2}}^{* 1}$ in the first variable while the line $B H$ is the axis of symmetry with respect to the adjoint $H_{\alpha_{1}, \alpha_{2}}^{* 2}$ in the second variable. But these adjoints are easily computed:

$$
\begin{aligned}
& H_{\alpha_{1}, \alpha_{2}}^{* 1}\left(f_{1}, f_{2}\right)=H_{-\alpha_{1}, \alpha_{2}-\alpha_{1}}\left(f_{1}, f_{2}\right), \\
& H_{\alpha_{1}, \alpha_{2}}^{* 2}\left(f_{1}, f_{2}\right)=H_{\alpha_{1}-\alpha_{2},-\alpha_{2}}\left(f_{1}, f_{2}\right) .
\end{aligned}
$$

Thus reflection across the axis $B H$ preserves boundedness for $\alpha_{2} / \alpha_{1}$ near 0 and interchanges boundedness for $\alpha_{2} / \alpha_{1}$ near 1 and $\infty$. Also reflection across the axis $C G$ preserves boundedness for $\alpha_{2} / \alpha_{1}$ near $\infty$ and interchanges boundedness for $\alpha_{2} / \alpha_{1}$ near 1 and 0 . Finally reflection across the axis $O F$ preserves boundedness for $\alpha_{2} / \alpha_{1}$ near 1 and interchanges boundedness for $\alpha_{2} / \alpha_{1}$ near 0 and $\infty$.

Using Theorem 1 above, Theorem 1 in [8], and bilinear interpolation we obtain that $H_{\alpha_{1}, \alpha_{2}}$ is bounded uniformly whenever $\alpha_{2} / \alpha_{1}$ is near 0 and $\infty$ in the open rectangle $D G H E$. We also obtain conclusion (1.5).

Duality with respect to $B H$ gives that $H_{\alpha_{1}, \alpha_{2}}$ is bounded uniformly whenever $\alpha_{2} / \alpha_{1}$ is near 0 and 1 in the interior of the rectangle $K F H L$. Similarly, duality with respect to $C G$ gives that $H_{\alpha_{1}, \alpha_{2}}$ is bounded uniformly whenever $\alpha_{2} / \alpha_{1}$ is near $\infty$ and 1 in the interior of the rectangle $L G F M$. Thus we obtain uniform bounds near all bad directions in the interior of the hexagon $K G L H M F$. Finally (1.3) and (1.4) follow from (1.5) using duality with respect to the axes $B H$ and $C G$.

Note that by a simple change of variables the boundedness of $H_{\alpha_{1}, \alpha_{2}}$ reduces to the case $\alpha_{1}=1$. It is easy to see that the boundedness of the operator $H_{1,-\alpha}$ on any product of Lebesgue spaces is equivalent to that of the bilinear operator

$$
\left(f_{1}, f_{2}\right) \rightarrow \int_{\mathbb{R}} \int_{\mathbb{R}} \widehat{f}_{1}(\xi) \widehat{f}_{2}(\eta) e^{2 \pi i(\xi+\eta) x} 1_{\left\{\eta<\alpha^{-1} \xi\right\}}(\xi, \eta) d \xi d \eta
$$

where $1_{A}$ denotes the characteristic function of the set $A$.

Therefore, for a positive integer $m$, we consider the following pseudodifferential operator

$$
T_{m}\left(f_{1}, f_{2}\right)(x)=\int_{\mathbb{R}} \int_{\mathbb{R}} \widehat{f}_{1}(\xi) \widehat{f}_{2}(\eta) e^{2 \pi i(\xi+\eta) x} 1_{\left\{\eta<2^{m} \xi\right\}}(\xi, \eta) d \xi d \eta .
$$

We prove the following result pertaining it. 
Let $1<p_{1}, p_{2}<2, \frac{2}{3}<p=\frac{p_{1} p_{2}}{p_{1}+p_{2}}<1$, and $p_{1} p_{2}>2$. Then there is a constant $C=C\left(p_{1}, p_{2}\right)$ such that for all $f_{1}, f_{2}$ Schwartz functions on $\mathbb{R}$ we have

$$
\left\|T_{m}\left(f_{1}, f_{2}\right)\right\|_{p} \leq C\left\|f_{1}\right\|_{p_{1}}\left\|f_{2}\right\|_{p_{2}}
$$

uniformly in $m \geq 2^{200}$.

By symmetry, it is easy to see that estimate (1.7) implies Theorem 1. We therefore only need to prove the former. In the sequel we will adopt the terminology and notation introduced in [8].

Acknowledgments. This article is part of the author's doctoral dissertation. The author would like to express his gratitude to his advisor, Loukas Grafakos, for his constructive suggestions, for his helpful advice and guidance, for his teaching, encouragement, and friendship. The author is very grateful to Christoph Thiele for his generous comments and suggestions. He is also thankful to Michael Lacey for the very helpful discussions during a visit at the Georgia Institute of Technology in 1998.

\section{The truncated trilinear form}

By the decomposition of the half plane $\eta<2^{m} \xi$ on the $\xi-\eta$ plane in [8], we only need to consider the following operator. (See [8] for the details.)

$$
T_{m}^{0}\left(f_{1}, f_{2}\right)(x)=\sum_{k \in \mathbb{Z}} \sum_{l \in \mathbb{Z}} \int_{\mathbb{R}} \int_{\mathbb{R}} \widehat{f_{1}}(\xi) \widehat{f_{2}}(\eta) e^{2 \pi i(\xi+\eta) x} \widehat{\Phi_{1, k, l}}(\xi) \widehat{\Phi_{2, k, l}}(\eta) d \xi d \eta .
$$

where $\Phi_{1, k, l}$ and $\Phi_{2, k, l}$ are suitable functions satisfying the following properties:

$$
\begin{aligned}
& \left|D^{\alpha} \widehat{\Phi_{1, k, l}}(\xi)\right| \leq C 2^{\alpha k}, \operatorname{supp} \widehat{\Phi_{1, k, l}} \subseteq\left(1+2^{-2 L}\right) J_{1}, \text { and } \\
& \widehat{\Phi_{1, k, l}}(\xi)=1 \text { for } \xi \in\left(1-2^{-2 L}\right) J_{1} \text {, where } J_{1}=\left[2^{-k} l, 2^{-k}(l+1)\right] . \\
& \left|D^{\alpha} \widehat{\Phi_{2, k, l}}(\xi)\right| \leq C 2^{\alpha(k-m)}, \operatorname{supp} \widehat{\Phi_{2, k, l}} \subseteq\left(1+2^{-2 L}\right) J_{2} \text {, and } \\
& \widehat{\Phi_{2, k, l}}(\xi)=1 \text { for } \xi \in\left(1-2^{-2 L}\right) J_{2}, \\
& \text { where } J_{2}=\left[2^{-k+m}(l-2), 2^{-k+m}(l-1)\right] \text { if } l \text { is even, } \\
& J_{2}=\left[2^{-k+m}(l-2), 2^{-k+m}(l-1)\right] \text { or }\left[2^{-k+m}(l-3), 2^{-k+m}(l-2)\right]
\end{aligned}
$$

if $l$ is odd, for all nonnegative integers $\alpha$. Note that the function $\Phi_{2, k, l}$ also depends on the parameter $m$, but this dependence will be suppressed for notational convenience. 
If follows from (2.2) and (2.3) that we have the following size estimates for the functions $\Phi_{1, k, l}$ and $\Phi_{2, k, l}$.

$$
\begin{aligned}
& \left|\Phi_{1, k, l}(x)\right| \leq \frac{C_{N} 2^{-k}}{\left(1+2^{-k}|x|\right)^{N}}, \\
& \left|\Phi_{2, k, l}(x)\right| \leq \frac{C_{N} 2^{-k+m}}{\left(1+2^{-k+m}|x|\right)^{N}}
\end{aligned}
$$

for any $N \in \mathbb{Z}^{+}$. The following lemma is also a consequence of $(2.2)$ and (2.3).

Lemma 1. For all $N \in \mathbb{Z}^{+}$and $1<p \leq 2$, there exists $C_{N}>0$ such that for all $f \in \mathcal{S}(\mathbb{R})$, we have

$$
\begin{aligned}
& \left(\sum_{l \in \mathbb{Z}}\left|\left(f * \Phi_{1, k, l}\right)(x)\right|^{p^{\prime}}\right)^{\frac{1}{p^{\prime}}} \leq C_{N}\left(\int|f(y)|^{p} \frac{2^{-k}}{\left(1+2^{-k}|x-y|\right)^{N}} d y\right)^{\frac{1}{p}} \\
& \left(\sum_{l \in \mathbb{Z}}\left|\left(f * \Phi_{2, k, l}\right)(x)\right|^{p^{\prime}}\right)^{\frac{1}{p^{\prime}}} \leq C_{N}\left(\int|f(y)|^{p} \frac{2^{-k+m}}{\left(1+2^{-k+m}|x-y|\right)^{N}} d y\right)^{\frac{1}{p}}
\end{aligned}
$$

where $C_{N}$ is independent of $m$.

Proof. We only prove (2.6). The proof of (2.7) is similar. In [8], we have proved the following inequality,

$$
\left(\sum_{l \in \mathbb{Z}}\left|\left(f * \Phi_{1, k, l}\right)(x)\right|^{2}\right)^{\frac{1}{2}} \leq C_{N}\left(\int|f(y)|^{2} \frac{2^{-k}}{\left(1+2^{-k}|x-y|\right)^{N}} d y\right)^{\frac{1}{2}}
$$

Note that, by (2.4), we have

$$
\sup _{l \in \mathbb{Z}}\left|\left(f * \Phi_{1, k, l}\right)(x)\right| \leq C_{N} \int|f(y)| \frac{2^{-k}}{\left(1+2^{-k}|x-y|\right)^{N}} d y .
$$

Then by Riesz-Thorin interpolation we obtain (2.6).

Let $\psi$ be a nonnegative Schwartz function such that $\widehat{\psi}$ is supported in $[-1,1]$ and satisfies $\widehat{\psi}(0)=1$. Let $\psi_{k}(x)=2^{-k} \psi\left(2^{-k} x\right)$. For $E \subset \mathbb{R}$ and $k \in \mathbb{Z}$ define

$$
\begin{gathered}
E_{k}=\left\{x \in E: \operatorname{dist}\left(x, E^{c}\right) \geq 2^{k}\right\} \\
\psi_{1, k}(x)=\left(1_{\left(E_{k}\right)^{c}} * \psi_{k}\right)(x), \quad \psi_{2, k}(x)=\psi_{3, k}(x)=\psi_{1, k-m}(x) .
\end{gathered}
$$

Note that $\psi_{1, k}, \psi_{2, k}$, and $\psi_{3, k}$ depend on the set $E$ but for notational convenience we will suppress this dependence since we will be working with a 
fixed set $E$. Also note that the functions $\psi_{2, k}$ and $\psi_{3, k}$ depend on $m$ but this dependence will also be overlooked in terms of notation. The important thing is that our estimates will be independent of $m$. Also define

$$
\Lambda_{E}\left(f_{1}, f_{2}, f_{3}\right)=\sum_{k \in \mathbb{Z}} \sum_{l \in \mathbb{Z}} \int \prod_{j=1}^{3} \psi_{j, k}(x)\left(f_{j} * \Phi_{j, k, l}\right)(x) d x
$$

where for any nonnegative integer $\alpha, \Phi_{3, k, l}$ depends on $m$ and satisfies

$$
\begin{aligned}
& \left|D^{\alpha} \widehat{\Phi_{3, k, l}}(\xi)\right| \leq C 2^{\alpha(k-m)}, \text { supp } \widehat{\Phi_{3, k, l}} \subset\left(1+2^{-2 L}\right) J_{3}, \\
& \widehat{\Phi_{3, k, l}}(\xi)=1 \text {, if } \xi \in J_{3} \text {, where } J_{3}=-\left(1+2^{-2 L}\right) J_{1}-\left(1+2^{-2 L}\right) J_{2},
\end{aligned}
$$

for all nonnegative integers $\alpha$. It is easy to obtain the following size estimate for $\Phi_{3, k, l}$

$$
\left|\Phi_{3, k, l}(x)\right| \leq \frac{C 2^{-k+m}}{\left(1+2^{-k+m}|x|\right)^{N}}
$$

The following lemma shows that we only need to consider the truncated trilinear form (2.12). Because of the assumptions on the indices $p_{1}, p_{2}$, there exists a $1<p_{3}<2$ such that $\frac{1}{p_{1}}+\frac{1}{p_{2}}+\frac{1}{p_{3}}<2$ and $p_{1} p_{2}>p_{3}^{\prime}$. Fix such a $p_{3}$ throughout the rest of the paper.

Lemma 2. Let $1<p_{1}, p_{2}, p_{3}<2, \frac{1}{p_{1}}+\frac{1}{p_{2}}+\frac{1}{p_{3}}<2, p_{1} p_{2}>p_{3}^{\prime}$, and $f_{j} \in \mathcal{S}$ with $\left\|f_{j}\right\|_{p_{j}}=1$ for $j \in\{1,2,3\}$. Define

$$
E=\bigcup_{j=1}^{3}\left\{x \in \mathbb{R}: M_{p_{j}}\left(M f_{j}\right)(x)>2\right\} .
$$

Then

$$
\left|\Lambda_{E}\left(f_{1}, f_{2}, f_{3}\right)\right| \leq C
$$

where $C$ is independent of $m$.

We will prove Lemma 2 in the next sections. Now we prove estimate (1.7) using Lemma 2.

Proof. To prove estimate (1.7), it is sufficient to prove that for all $\lambda>0$ we have

$$
\left|\left\{x:\left|T_{m}^{0}\left(f_{1}, f_{2}\right)(x)\right|>\lambda\right\}\right| \leq \frac{C}{\lambda^{\frac{p_{1} p_{2}}{p_{1}+p_{2}}}}
$$

whenever $\left\|f_{1}\right\|_{p_{1}}=\left\|f_{2}\right\|_{p_{2}}=1$. By linearity and scale invariance, it suffices to prove that

$$
\left|\left\{x:\left|T_{m}^{0}\left(f_{1}, f_{2}\right)(x)\right|>2\right\}\right| \leq C .
$$


Let $E=\bigcup_{j=1}^{2}\left\{x \in \mathbb{R}: M_{p_{j}}\left(M f_{j}\right)(x)>2\right\}$. Observe that $|E| \leq C$, therefore it is enough to show that

$$
\left|\left\{x \in E^{c}:\left|T_{m}^{0}\left(f_{1}, f_{2}\right)(x)\right|>2\right\}\right| \leq C .
$$

Let $G=\left\{x \in E^{c}:\left|T_{m}^{0}\left(f_{1}, f_{2}\right)(x)\right|>2\right\}$, and choose $f_{3} \in \mathcal{S}$ with $\left\|f_{3}\right\|_{L^{\infty}\left(E^{c}\right)} \leq 1, \operatorname{supp} f_{3} \subseteq E^{c}$, and

$$
\left\|f_{3}(\cdot)-\frac{1_{G}(\cdot)}{|G|^{1 / p_{3}}} \frac{T_{m}^{0}\left(f_{1}, f_{2}\right)(\cdot)}{\left|T_{m}^{0}\left(f_{1}, f_{2}\right)(\cdot)\right|}\right\|_{p_{3}} \leq \min \left\{1,\left\|T_{m}^{0}\left(f_{1}, f_{2}\right)\right\|_{p_{3}^{\prime}}^{-1}\right\} .
$$

Note that for the $f_{3}$ chosen we have $\left\|f_{3}\right\|_{p_{3}} \leq 2$ and thus the set $\{x \in \mathbb{R}$ : $\left.M_{p_{3}}\left(M f_{3}\right)(x)>2\right\}$ is empty. Now define

$$
\Lambda\left(f_{1}, f_{2}, f_{3}\right)=\sum_{k \in \mathbb{Z}} \sum_{l \in \mathbb{Z}} \int \prod_{j=1}^{3}\left(f_{j} * \Phi_{j, k, l}\right)(x) d x .
$$

Then by Lemma 2 it follows that

$$
\begin{aligned}
|G|^{1 / p_{3}^{\prime}} & \leq\left\langle T_{m}^{0}\left(f_{1}, f_{2}\right), \frac{1_{G}(\cdot)}{|G|^{1 / p_{3}}} \frac{T_{m}^{0}\left(f_{1}, f_{2}\right)(\cdot)}{\left|T_{m}^{0}\left(f_{1}, f_{2}\right)(\cdot)\right|}\right\rangle \\
& \leq\left|\left\langle T_{m}^{0}\left(f_{1}, f_{2}\right), f_{3}-\frac{1_{G}(\cdot)}{|G|^{1 / p_{3}}} \frac{T_{m}^{0}\left(f_{1}, f_{2}\right)(\cdot)}{\left|T_{m}^{0}\left(f_{1}, f_{2}\right)(\cdot)\right|}\right\rangle\right|+\left|\Lambda\left(f_{1}, f_{2}, f_{3}\right)\right| \\
& \leq C+\left|\Lambda\left(f_{1}, f_{2}, f_{3}\right)-\Lambda_{E}\left(f_{1}, f_{2}, f_{3}\right)\right|+\left|\Lambda_{E}\left(f_{1}, f_{2}, f_{3}\right)\right| \\
& \leq\left|\Lambda\left(f_{1}, f_{2}, f_{3}\right)-\Lambda_{E}\left(f_{1}, f_{2}, f_{3}\right)\right|+C .
\end{aligned}
$$

Thus, to prove (2.16), we only need to show that

$$
\left|\Lambda\left(f_{1}, f_{2}, f_{3}\right)-\Lambda_{E}\left(f_{1}, f_{2}, f_{3}\right)\right| \leq C
$$

whenever $\left\|f_{3}\right\|_{L^{\infty}\left(E^{c}\right)} \leq 1$ and $\operatorname{supp} f_{3} \subseteq E^{c}$. We now prove (2.18). We clearly have

$$
\begin{aligned}
\mid \Lambda\left(f_{1}, f_{2}, f_{3}\right) & -\Lambda_{E}\left(f_{1}, f_{2}, f_{3}\right) \mid \\
& \leq\left|\sum_{k \in \mathbb{Z}} \sum_{l \in \mathbb{Z}} \int\left(1-\prod_{j=1}^{3} \psi_{j, k}(x)\right) \prod_{j=1}^{3}\left(f_{j} * \Phi_{j, k, l}\right)(x) d x\right| .
\end{aligned}
$$

But recall that $\psi_{2, k}=\psi_{3, k}$, hence

$$
\left|1-\prod_{j=1}^{3} \psi_{j, k}(x)\right| \leq\left|1-\psi_{1, k}(x)\right|+2\left|1-\psi_{2, k}(x)\right| .
$$


Thus the expression on the right in (2.19) is at most equal to the sum of the following two quantities

$$
\begin{aligned}
& \sum_{k \in \mathbb{Z}} \int\left|1-\psi_{1, k}(x)\right| \sum_{l \in \mathbb{Z}} \prod_{j=1}^{3}\left|\left(f_{j} * \Phi_{j, k, l}\right)(x)\right| d x, \\
& 2 \sum_{k \in \mathbb{Z}} \int\left|1-\psi_{2, k}(x)\right| \sum_{l \in \mathbb{Z}} \prod_{j=1}^{3}\left|\left(f_{j} * \Phi_{j, k, l}\right)(x)\right| d x .
\end{aligned}
$$

Observe that $\sum_{l \in \mathbb{Z}} \prod_{j=1}^{3}\left|\left(f_{j} * \Phi_{j, k, l}\right)(x)\right|$ is smaller than

$$
\left(\sum_{l \in \mathbb{Z}}\left|\left(f_{1} * \Phi_{1, k, l}\right)(x)\right|^{p_{1}^{\prime}}\right)^{\frac{1}{p_{1}^{\prime}}}\left(\sum_{l \in \mathbb{Z}}\left|\left(f_{2} * \Phi_{2, k, l}\right)(x)\right|^{p_{2}^{\prime}}\right)^{\frac{1}{p_{2}^{\prime}}}\left(\sum_{l \in \mathbb{Z}}\left|\left(f_{3} * \Phi_{3, k, l}\right)(x)\right|^{q^{\prime}}\right)^{\frac{1}{q^{\prime}}},
$$

where $q$ satisfies $\frac{1}{p_{1}^{\prime}}+\frac{1}{p_{2}^{\prime}}+\frac{1}{q^{\prime}}=1$.

Using (2.6) and the fact that $1<p_{1}<2$, for any point $z_{0} \in E^{c}$, we obtain

$$
\begin{aligned}
\left(\sum_{l \in \mathbb{Z}}\left|\left(f_{1} * \Phi_{1, k, l}\right)(x)\right|^{p_{1}^{\prime}}\right)^{\frac{1}{p_{1}^{\prime}}} \\
\quad \leq C\left(\int\left|f_{1}(y)\right|^{p_{1}} \frac{2^{-k}\left(1+2^{-k}\left|x-z_{0}\right|\right)^{2}}{\left(1+2^{-k}|x-y|\right)^{N}\left(1+2^{-k}\left|x-z_{0}\right|\right)^{2}} d y\right)^{\frac{1}{p_{1}}} \\
\quad \leq C\left(\int\left|f_{1}(y)\right|^{p_{1}} \frac{2^{-k}\left(1+2^{-k}\left|x-z_{0}\right|\right)^{2}}{\left(1+2^{-k}\left|y-z_{0}\right|\right)^{2}} d y\right)^{\frac{1}{p_{1}}} \\
\leq C\left(1+2^{-k}\left|x-z_{0}\right|\right)^{\frac{2}{p_{1}}} M_{p_{1}} f_{1}\left(z_{0}\right) \\
\leq C\left(1+2^{-k} \operatorname{dist}\left(x, E^{c}\right)\right)^{2} .
\end{aligned}
$$

Similarly, using (2.7) and the fact that $1<p_{2}, q<2$ we obtain

$$
\left(\sum_{l \in \mathbb{Z}}\left|\left(f_{2} * \Phi_{2, k, l}\right)(x)\right|^{p_{2}^{\prime}}\right)^{\frac{1}{p_{2}^{\prime}}} \leq C\left(1+2^{-k+m} \operatorname{dist}\left(x, E^{c}\right)\right)^{2} .
$$

Using (2.14) and the facts that $\left\|f_{3}\right\|_{L^{\infty}\left(E^{c}\right)} \leq 1$ and supp $f_{3} \subseteq E^{c}$, we also obtain

$$
\begin{aligned}
& \left(\sum_{l \in \mathbb{Z}}\left|\left(f_{3} * \Phi_{3, k, l}\right)(x)\right|^{q^{\prime}}\right)^{\frac{1}{q^{\prime}}} \\
& \quad \leq C\left(\int\left|f_{3}(y)\right|^{q} \frac{2^{-k+m}}{\left(1+2^{-k+m}|x-y|\right)^{N}} d y\right)^{\frac{1}{q}} \leq \frac{C}{\left(1+2^{-k+m} \operatorname{dist}\left(x, E^{c}\right)\right)^{N}}
\end{aligned}
$$


Therefore, (2.20) can be estimated by

$$
\begin{aligned}
& C \sum_{k} \iint_{E_{k}} \frac{2^{-k}}{\left(1+2^{-k}|x-y|\right)^{N}} d y \frac{1}{\left(1+2^{-k+m} \operatorname{dist}\left(x, E^{c}\right)\right)^{N-2}} d x \\
& \quad \leq C \sum_{k} \int_{E_{k}} \frac{1}{\left(1+2^{-k} \operatorname{dist}\left(y, E^{c}\right)\right)^{N-2}} d y \\
& \quad \leq C \int_{E} \sum_{\substack{k \in \mathbb{Z} \\
2^{k} \leq \operatorname{dist}\left(y, E^{c}\right)}} \frac{1}{\left(1+2^{-k} \operatorname{dist}\left(y, E^{c}\right)\right)^{N-2}} d y \leq C|E| \leq C .
\end{aligned}
$$

Similarly (2.21) is estimated by

$$
\begin{aligned}
& C \sum_{k} \iint_{E_{k-m}} \frac{2^{-k+m}}{\left(1+2^{-k+m}|x-y|\right)^{N}} d y \frac{1}{\left(1+2^{-k+m} \operatorname{dist}\left(x, E^{c}\right)\right)^{N-2}} d x \\
& \quad \leq C \sum_{k} \int_{E_{k-m}} \frac{1}{\left(1+2^{-k+m} \operatorname{dist}\left(y, E^{c}\right)\right)^{N-2}} d y \\
& \quad \leq C \int_{E} \sum_{\substack{k \in \mathbb{Z} \\
2^{k} \leq \operatorname{dist}\left(y, E^{c}\right)}} \frac{1}{\left(1+2^{-k} \operatorname{dist}\left(y, E^{c}\right)\right)^{N-2}} d y \leq C|E| \leq C .
\end{aligned}
$$

This completes the proof of (2.18) and therefore of estimate (1.7).

We will now set up some notation. For $k, n \in \mathbb{Z}$, define $I_{k, n}=\left[2^{k} n, 2^{k}(n+1)\right]$. And let

$$
\begin{aligned}
& \phi_{1, k, n}(x)=\left(1_{I_{k, n}} * \psi_{k}\right)(x), \\
& \phi_{j, k, n}(x)=\left(1_{I_{k, n}} * \psi_{k-m}\right)(x), \quad \text { when } j \in\{2,3\} .
\end{aligned}
$$

Therefore we can write

$$
\Lambda_{E}\left(f_{1}, f_{2}, f_{3}\right)=\sum_{k \in \mathbb{Z}} \sum_{l \in \mathbb{Z}} \int \prod_{j=1}^{3}\left(\sum_{n \in \mathbb{Z}} \phi_{j, k, n}(x) \psi_{j, k}(x)\left(f_{j} * \Phi_{j, k, l}\right)(x)\right) d x .
$$

For an integer $r$ with $0 \leq r<L$, let $\mathbb{Z}_{r}=\{\ell \in \mathbb{Z}: \ell=\kappa L+r$ for some $\kappa \in \mathbb{Z}\}$. Also for $S \subset \mathbb{Z}_{r} \times \mathbb{Z} \times \mathbb{Z}_{r}$ we let $S_{k, l}=\{n \in \mathbb{Z}:(k, n, l) \in S\}$ and we define

$$
\Lambda_{E, S}\left(f_{1}, f_{2}, f_{3}\right)=\sum_{k \in \mathbb{Z}_{r}} \sum_{l \in \mathbb{Z}_{r}} \int \prod_{j=1}^{3}\left(\sum_{n \in S_{k, l}} \phi_{j, k, n}(x) \psi_{j, k}(x)\left(f_{j} * \Phi_{j, k, l}\right)(x)\right) d x .
$$


For simplicity we will only consider the case where $m \in \mathbb{Z}_{0}$. There is no difficulty in adjusting the argument below to the case where $m$ has a different remainder when divided by $L$. We will therefore concentrate in proving Lemma 2 for the expression $\Lambda_{E, S}\left(f_{1}, f_{2}, f_{3}\right)$ when $m \in \mathbb{Z}_{0}$. To achieve this goal, we recall the grid structure introduced in Definition 1 in [8].

When $S \subset \mathbb{Z}_{r} \times \mathbb{Z} \times \mathbb{Z}_{r}$ and $s=(k, n, l) \in S$ we set $I_{s}=I_{k, n}$. Also for $j \in$ $\{1,2,3\}$, as in [8], we let $\omega_{j, s}$ be intervals such that conditions (2.25)-(2.31) below hold:

$$
\begin{gathered}
\left|c\left(\omega_{1, s}\right)-2^{-k}\left(l+\frac{1}{2}\right)\right| \leq 5 \cdot 2^{-L} 2^{-k}, \\
\left|c\left(\omega_{2, s}\right)-2^{-k+m}\left(l-\frac{3}{2}\right)\right| \leq 5 \cdot 2^{-L} 2^{-k+m} \text { and } \omega_{2, s}=\omega_{3, s}, \\
\operatorname{supp} \widehat{\Phi_{j, k, l}} \subset \omega_{j, s} \text { for } j \in\{1,2\}, \\
\operatorname{supp} \widehat{\Phi_{3, k, l}} \subset\left[-\left(1+2^{-m}\right) a,-\left(1+2^{-m}\right) b\left[\text { , where } \left[a, b\left[=\omega_{3, s},\right.\right.\right.\right. \\
\left(1+2^{-2 L}\right) 2^{-k} \leq\left|\omega_{1, s}\right| \leq\left(1+10 \cdot 2^{-L}\right) 2^{-k}, \\
\left(1+2^{-2 L}\right) 2^{-k+m} \leq\left|\omega_{j, s}\right| \leq\left(1+2 \cdot 2^{-2 L}\right)\left(1+5 \cdot 2^{-L}\right) 2^{-k+m} \\
\text { for } j \in\{2,3\}, \\
\left\{\omega_{j, s}\right\}_{s \in S} \text { is a central grid, for } j \in\{1,2,3\} .
\end{gathered}
$$

Furthermore, we have the following geometric picture for $\omega_{j, s}$, which has proved in [8].

Lemma 3. For $s, s^{\prime} \in S$ and $\omega_{j, s} \neq \omega_{j, s^{\prime}}$, the following properties hold

(1) If $\omega_{1, s} \subset \omega_{1, s^{\prime}}$, then $\omega_{j, s^{\prime}}<\omega_{j, s}$ and $\frac{1}{2}\left|\omega_{j, s^{\prime}}\right|<\operatorname{dist}\left(\omega_{j, s}, \omega_{j, s^{\prime}}\right)<2\left|\omega_{2, s^{\prime}}\right|$ for $j=2,3$.

(2) If $\omega_{j, s} \subset \omega_{j, s^{\prime}}$ for $j=2,3$, then $\omega_{1, s}<\omega_{1, s^{\prime}}$ and $\frac{1}{8}\left|\omega_{1, s^{\prime}}\right|<\operatorname{dist}\left(\omega_{1, s}, \omega_{1, s^{\prime}}\right)$ $<2\left|\omega_{1, s^{\prime}}\right|$.

As in [8] and [14] we give the following definition.

Definition 1. A subset $S$ of $\mathbb{Z}_{r} \times \mathbb{Z} \times \mathbb{Z}_{r}$ is called convex if for all $s, s^{\prime \prime} \in S$, $s^{\prime} \in \mathbb{Z}_{r} \times \mathbb{Z} \times \mathbb{Z}_{r}, j \in\{1,2\}$ with $I_{s} \subset I_{s^{\prime}} \subset I_{s^{\prime \prime}}$ and $\omega_{j, s^{\prime \prime}} \subset \omega_{j, s^{\prime}} \subset \omega_{j, s}$, we have $s^{\prime} \in S$.

It is sufficient to obtain bounds for $\Lambda_{E, S}$ for all finite convex sets $S$ of triples of integers, provided the bound is independent of $S$ and of course $m$. 


\section{The selection of the trees}

In this section, we start the proof of Lemma 2. We begin with the following

Definition 2. Fix $T \subset S$ and $t \in T$. If for any $s \in T$, we have $I_{s} \subset I_{t}$ and $\omega_{j, s} \supset \omega_{j, t}$, then we call $T$ a tree of type $j$ with top $t . T$ is called a maximal tree of type $j \in\{1,2\}$ with top $t$ in $S$ if there does not exist a larger tree of type $j$ with the same top strictly containing $T$. Let $T$ be a maximal tree of type $j \in\{1,2\}$ with top $t$ in $S$, and $i \in\{1,2\}, i \neq j$. Denote the maximal tree of type $i$ with top $t$ in $S$ by $\widetilde{T}$.

We recall some notation from [8] needed in the selection of the trees that follows. For a given subset $T$ of $S$ we define $T_{k, l}$ to be the set $\{n \in \mathbb{Z}$ : $(k, n, l) \in T\}$. If $T$ is a tree of type $j$ for $j \in\{1,2,3\}$ and $k \in \mathbb{Z}_{r}$, then there is at most one $l \in \mathbb{Z}_{r}$ such that $T_{k, l} \neq \emptyset$. If such an $l$ exists, then let $T_{k}=T_{k, l}$ and $\Phi_{j, k, T}=\Phi_{j, k, l}$. Otherwise, let $T_{k}=\emptyset$ and $\Phi_{j, k, T}=0$. For brevity, we write $(k, n) \in T$ if and only if there exists an $l \in \mathbb{Z}_{r}$ with $(k, n, l) \in T$. Therefore, if $(k, n, l) \in T$, we can write $\omega_{j, k, n, l}=\omega_{j, k, l}=\omega_{j, k, T}$, and

$$
\Lambda_{E, T}\left(f_{1}, f_{2}, f_{3}\right)=\sum_{k \in \mathbb{Z}_{r}} \int \prod_{j=1}^{3}\left(\sum_{n \in T_{k}} \phi_{j, k, n}(x) \psi_{j, k}(x)\left(f_{j} * \Phi_{j, k, T}\right)(x)\right) d x
$$

Let $t=\left(k_{T}, n_{T}, l_{T}\right)$ be the top of $T$. We write $I_{T}=I_{k_{T}, n_{T}}$ and $\omega_{j, T}=\omega_{j, k_{T}, T}$.

For a tree $T$ of type 2 (or 3 ) with top $t$ and $k \in \mathbb{Z}_{r}$, define $\theta_{j, k, T}^{+}$and $\theta_{j, k, T}^{-}$by

$$
\begin{aligned}
& \widehat{\theta_{j, k, T}^{+}}(\xi)=\left(\Phi_{j, k-L, T}-\Phi_{j, k, T}\right)^{\wedge}(\xi) 1_{\xi \geq \alpha_{j} c\left(w_{j, t}\right)}(\xi), \\
& \widehat{\theta_{j, k, T}^{-}}(\xi)=\left(\Phi_{j, k-L, T}-\Phi_{j, k, T}\right)^{\wedge}(\xi) 1_{\xi \leq \alpha_{j} c\left(w_{j, t}\right)}(\xi),
\end{aligned}
$$

where $\alpha_{j}=1$ if $j=2$ and $\alpha_{j}=1+2^{-m}$, if $j=3$. Let $\psi^{*}(x)=\left(1+x^{2}\right)^{-N}$. In accordance with the definitions of $\phi_{j, k, n}$ and $\psi_{j, k}$ we define the functions

$$
\begin{aligned}
& \psi_{1, k}^{*}(x)=\left(1_{\left(E_{k}\right)^{c}} * \psi_{k}^{*}\right)(x), \\
& \psi_{j, k}^{*}(x)=\psi_{1, k-m}^{*}(x), \quad \text { when } j \in\{2,3\} .
\end{aligned}
$$

and

$$
\begin{aligned}
\phi_{1, k, n}^{*}(x) & =\left(1_{I_{k, n}} * \psi_{k}^{*}\right)(x), \\
\phi_{j, k, n}^{*}(x) & =\left(1_{I_{k, n}} * \psi_{k-m}^{*}\right)(x), \quad \text { when } j \in\{2,3\} .
\end{aligned}
$$

Let $\Delta_{k}$ be the set of all connected components of $E_{k} \backslash E_{k+L}$. Obviously $\Delta_{k}$ is a set of intervals. Observe that if $J \in \Delta_{k}$, then $2^{k} \leq|J|<2^{k+L}$, and $\bigcup_{k} \Delta_{k}$ is a set of pairwise disjoint intervals. 


\section{Define}

$$
\Delta_{k, T}=\left\{J \in \Delta_{k}: J \subset I_{k+m+L, n}, \text { for some }(k+m+L, n) \in T\right\},
$$

and for $J \in \Delta_{k, T}$ define

$$
\rho_{k, J}(x)=1_{J} * \psi_{k}^{*}(x) \text {, where } \psi_{k}^{*}(x)=2^{-k} \psi^{*}\left(2^{-k} x\right) .
$$

Throughout this paper fix $0<\eta \leq L^{-1}\left(\frac{p_{2}}{p_{3}^{\prime}}-\frac{1}{p_{1}}\right) \min _{j \in\{1,2,3\}}\left\{\frac{1}{p_{j}^{\prime}}\right\}$ and let $H$ be the set

$$
\bigcup_{j=1}^{3}\{(1, j, 1),(2,1,1),(3,1,1)\} \bigcup \bigcup_{\nu=2}^{5}\{(2,2, \nu),(2,3, \nu),(3,2, \nu),(3,3, \nu)\} .
$$

We now describe a procedure for selecting a collection of trees $T_{\mu, i, j, l}^{\nu}$ and $\widetilde{T}_{\mu, i, j, l}^{\nu}$ by induction on $\mu$ and $l$. Let $S_{-1}=S$, and for $\mu \geq 0$ let

$$
S_{\mu}=S_{\mu-1} \backslash \bigcup_{(i, j, \nu) \in H} \bigcup_{l \geq 0}\left(T_{\mu, i, j, l}^{\nu} \cup \widetilde{T}_{\mu, i, j, l}^{\nu}\right)
$$

where $T_{\mu, i, j, l}^{\nu}, \widetilde{T}_{\mu, i, j, l}^{\nu}$ are defined as follows:

Let $l \geq 0$ be an integer and assume that we have already defined $T_{\mu, i, j, \lambda}^{\nu}$, $\widetilde{T}_{\mu, i, j, \lambda}^{\nu}$ for $\lambda<l$. If one of the sets $T_{\mu, i, j, \lambda}^{\nu}, \widetilde{T}_{\mu, i, j, \lambda}^{\nu}$ with $\lambda<l$ is empty, then let $T_{\mu, i, j, l}^{\nu}=\widetilde{T}_{\mu, i, j, l}^{\nu}=\emptyset$. Otherwise, let $\mathcal{F}$ denote the set of all trees $T$ of type $i$ satisfying the following conditions (1)-(8).

(1) For $(i, j, \nu) \in H$,

$$
T \subset S_{\mu-1} \backslash \bigcup_{\lambda<l}\left(T_{\mu, i, j, \lambda}^{\nu} \cup \widetilde{T}_{\mu, i, j, \lambda}^{\nu}\right)
$$

and $T$ is a maximal tree of type $i$ in $S_{\mu-1} \backslash \bigcup_{\lambda<l}\left(T_{\mu, i, j, \lambda}^{\nu} \cup \widetilde{T}_{\mu, i, j, \lambda}^{\nu}\right)$.

(2) If $(i, j, \nu)=(1,1,1)$, then for $(k, n) \in T$, one of the following inequalities holds:

$$
\begin{gathered}
\left\|\phi_{1, k, n}^{*} \psi_{1, k}^{*}\left(f_{1} * \Phi_{1, k, l}\right)\right\|_{p_{1}} \geq 2^{-\eta \mu} 2^{-\frac{\mu}{p_{1}^{\prime}}}\left|I_{k, n}\right|^{\frac{1}{p_{1}}} \\
\left\|\phi_{1, k, n}^{*} \psi_{1, k}^{*}\left(e^{-2 \pi i c\left(\omega_{1, k, l}\right)(\cdot)}\left(f_{1} * \Phi_{1, k, l}\right)(\cdot)\right)^{\prime}\right\|_{p_{1}} \geq 2^{-\eta \mu} 2^{-\frac{\mu}{p_{1}^{\prime}}}\left|I_{k, n}\right|^{\frac{1}{p_{1}}-1} .
\end{gathered}
$$

(3) If $(i, j, \nu)=(1,2,1)$ or $(1,3,1)$, then

$$
\left\|\left(\sum_{(k, n) \in T}\left|\phi_{j, k, n}^{*} \psi_{j, k}^{*}\left(f_{j} * \Phi_{j, k, T}\right)\right|^{2}\right)^{\frac{1}{2}}\right\|_{p_{j}} \geq 2^{4} 2^{-\frac{\mu}{p_{j}^{\prime}}}\left|I_{T}\right|^{\frac{1}{p_{j}}} .
$$


(4) If $(i, j, \nu)=(2,1,1)$ or $(3,1,1)$, then one of the following inequalities holds:

$$
\left\|\left(\sum_{(k, n) \in T}\left|\phi_{1, k, n}^{*} \psi_{1, k}^{*}\left(f_{1} * \Phi_{1, k, T}\right)\right|^{2}\right)^{\frac{1}{2}}\right\|_{p_{1}} \geq 2^{4} 2^{-\frac{\mu}{p_{1}^{\prime}}}\left|I_{T}\right|^{\frac{1}{p_{1}}}
$$

(5) If $i=2$ or $3, j=2$ or $3, \nu=2$, then there exists $\tilde{k} \in\{-L, 0, L, 2 L, 3 L, 4 L\}$ such that, for $(k, n) \in T$, one of the following inequalities holds:

$$
\begin{gathered}
\left\|\phi_{j, k+\tilde{k}, n}^{*} \psi_{j, k+\tilde{k}}^{*}\left(f_{j} * \Phi_{j, k+\tilde{k}, l}\right)\right\|_{p_{j}} \geq 2^{-\eta \mu} 2^{-\frac{\mu}{p_{j}^{\prime}}}\left|I_{k, n}\right|^{\frac{1}{p_{j}}} \\
\left\|\phi_{1, k, n}^{*} \psi_{j, k+m+\tilde{k}}^{*}\left(f_{j} * \Phi_{j, k+m+\tilde{k}, l}\right)\right\|_{p_{j}} \geq 2^{-\eta \mu} 2^{-\frac{\mu}{p_{j}^{\prime}}}\left|I_{k, n}\right|^{\frac{1}{p_{j}}} \\
\left\|\phi_{1, k, n}^{*} \psi_{j, k+m+\tilde{k}}^{*}\left(e^{-2 \pi i c\left(\omega_{j, k+m+\tilde{k}, T}\right)(\cdot)}\left(f_{j} * \Phi_{j, k+m+\tilde{k}, l}\right)(\cdot)\right)^{\prime}\right\|_{p_{j}} \\
\geq 2^{-\eta \mu} 2^{-\frac{\mu}{p_{j}^{\prime}}}\left|I_{k, n}\right|^{\frac{1}{p_{j}}-1} \cdot
\end{gathered}
$$

(6) If $i=2$ or $3, j=2$ or $3, \nu=3$, then

$$
\left\|\left(\sum_{(k, n) \in T}\left|\phi_{j, k, n}^{*} \psi_{j, k}^{*}\left(f_{j} * \theta_{j, k, T}^{+}\right)\right|^{2}\right)^{\frac{1}{2}}\right\|_{p_{j}} \geq 2^{4} 2^{-\frac{\mu}{p_{j}^{\prime}}}\left|I_{T}\right|^{\frac{1}{p_{j}}}
$$

(7) If $i=2$ or $3, j=2$ or $3, \nu=4$, then

$$
\left\|\left(\sum_{(k, n) \in T}\left|\phi_{j, k, n}^{*} \psi_{j, k}^{*}\left(f_{j} * \theta_{j, k, T}^{-}\right)\right|^{2}\right)^{\frac{1}{2}}\right\|_{p_{j}} \geq 2^{4} 2^{-\frac{\mu}{p_{j}^{\prime}}}\left|I_{T}\right|^{\frac{1}{p_{j}}} .
$$

(8) If $i=2$ or $3, j=2$ or $3, \nu=5$, then there exists $\tilde{k} \in\{-L, 0, L, 2 L, 3 L, 4 L\}$ such that

$$
\left\|\left(\sum_{k} \sum_{J \in \Delta_{k-m, T}}\left|\rho_{k-m, J}\left(f_{j} * \Phi_{j, k+\tilde{k}, T}\right)\right|^{2}\right)^{\frac{1}{2}}\right\|_{p_{j}} \geq 2^{4} 2^{-\frac{\mu}{p_{j}^{\prime}}}\left|I_{T}\right|^{\frac{1}{p_{j}}}
$$

If no such trees exist, in other words if $\mathcal{F}=\emptyset$, then we set $T_{\mu, i, j, l}^{\nu}=$ $\widetilde{T}_{\mu, i, j, l}^{\nu}=\emptyset$. Otherwise, we select $T_{\mu, i, j, l}^{\nu}$ and $\widetilde{T}_{\mu, i, j, l}^{\nu}$ as follows:

(9) If $(i, j, \nu) \in\{(1,2,1),(1,3,1),(2,2,4),(2,3,4),(3,2,4),(3,3,4)\}$, then select $T_{\mu, i, j, l}^{\nu} \in \mathcal{F}$ such that for any $T \in \mathcal{F}$ we have

$$
\omega_{j, T_{\mu, i, j, l}^{\nu}} \ngtr \omega_{j, T}
$$


Let $\widetilde{T}_{\mu, i, j, l}^{\nu}$ be the maximal tree of type $i^{\prime}$ with top $t$ in $S_{\mu-1} \backslash \bigcup_{\lambda<l}\left(T_{\mu, i, j, \lambda}^{\nu} \cup\right.$ $\left.\widetilde{T}_{\mu, i, j, \lambda}^{\nu}\right)$, where $i^{\prime}=2$ if $i=1, i^{\prime}=1$ if $i \in\{2,3\}$, and $t$ is the top of $T_{\mu, i, j, l}^{\nu}$.

(10) If $(i, j, \nu) \in\{(2,1,1),(3,1,1),(2,2,3),(2,3,3),(3,2,3),(3,3,3)\}$, then select $T_{\mu, i, j, l}^{\nu} \in \mathcal{F}$ such that for any $T \in \mathcal{F}$ we have

$$
\omega_{j, T_{\mu, i, j, l}^{\nu}} \nless \omega_{j, T}
$$

Let $\widetilde{T}_{\mu, i, j, l}^{\nu}$ be the maximal tree of type $i^{\prime}$ with top $t$ in $S_{\mu-1} \backslash \bigcup_{\lambda<l}\left(T_{\mu, i, j, \lambda}^{\nu} \cup\right.$ $\left.\widetilde{T}_{\mu, i, j, \lambda}^{\nu}\right)$, where $i^{\prime}=2$ if $i=1, i^{\prime}=1$ if $i \in\{2,3\}$, and $t$ is the top of $T_{\mu, i, j, l}^{\nu}$.

This completes the selection of trees. As in [8], it is easy to see that $S_{\mu}$, $T_{\mu, i, j, l}^{\nu}$ and $\widetilde{T}_{\mu, i, j, l}^{\nu}$ are convex.

Until the end of the paper we fix $1<q_{1}, q_{2}, q_{3}<\infty$ with $\frac{1}{q_{1}}+\frac{1}{q_{2}}+\frac{1}{q_{3}}=1$ such that $q_{1}$ is very large, and $q_{2}>p_{3}^{\prime}, q_{3}>p_{3}$.

The core of the proof is to obtain Lemmata 4 and 5 below which will be proved in the next sections.

Lemma 4. Let $\mu \geq 0, j \in\{1,2,3\}$, $T$ be a tree of type $j$ and $T \subset S_{\mu}$, then

$$
\left|\Lambda_{E, T}\left(f_{1}, f_{2}, f_{3}\right)\right| \leq C 2^{-\eta \mu_{2}} 2^{-\left(\frac{1}{p_{1}^{\prime}}+\frac{1}{p_{2}^{\prime}} \frac{p_{2}}{p_{3}^{\prime}}+\frac{1}{p_{3}^{\prime}}\right) \mu}\left|I_{T}\right| \quad \text { if } \quad j=1,
$$

And if $T$ is a convex set, then

$$
\left|\Lambda_{E, T}\left(f_{1}, f_{2}, f_{3}\right)\right| \leq C_{q_{1}} 2^{-\left(\frac{1}{p_{1}^{\prime}}+\frac{1}{p_{2}^{\prime}} \frac{p_{2}}{q_{2}}+\frac{1}{p_{3}^{\prime}} \frac{p_{3}}{q_{3}}\right) \mu}\left|I_{T}\right| \text { if } j=2,3,
$$

where $C, C_{q_{1}}$ are independent of $m$.

Lemma 5. For $\mu \geq 0,(i, j, \nu) \in H$,

$$
\sum_{l \geq 0}\left|I_{T_{\mu, i, j, l}^{\nu}}\right| \leq C 2^{10 \eta p_{j}^{\prime} \mu} 2^{\mu}
$$

where $C$ is independent of $m$.

Once Lemmata 4 and 5 are proved, then the only extra ingredient we need to polish off the proof of estimate (1.7) is the following lemma.

Lemma 6. Let $\mu \geq 0, T \subset S_{\mu-1}$ be a tree of type $j \in\{1,2,3\}, P \subset S_{\mu-1}$, and $T \cap P=\emptyset$. Suppose $T$ is a maximal tree in $T \cup P$. Then

$$
\begin{aligned}
\mid \Lambda_{E, T \cup P}\left(f_{1}, f_{2}, f_{3}\right) & -\Lambda_{E, P}\left(f_{1}, f_{2}, f_{3}\right) \mid \\
& \leq\left|\Lambda_{E, T}\left(f_{1}, f_{2}, f_{3}\right)\right|+C 2^{-\eta \mu-\left(\frac{1}{p_{1}^{\prime}}+\frac{1}{p_{2}^{\prime}} \frac{p_{2}}{p_{3}^{\prime}}+\frac{1}{p_{3}^{\prime}}\right) \mu}\left|I_{T}\right|,
\end{aligned}
$$

where $C$ is independent of $\mu, P, T$ and $m$. 
Proof. Notice there exists at most one $l$ such that $T_{k, l} \neq \emptyset$ and $T$ is a maximal tree in $T \cup P$, we have

$$
\left|\Lambda_{E, T \cup P}\left(f_{1}, f_{2}, f_{3}\right)-\Lambda_{E, P}\left(f_{1}, f_{2}, f_{3}\right)\right| \leq\left|\Lambda_{E, T}\left(f_{1}, f_{2}, f_{3}\right)\right|+\sum_{k \leq k_{T}} \int\left|A_{k}(x)\right| d x,
$$

where $\left|A_{k}\right|$ is controlled by

$$
\frac{C}{\left(1+2^{-k} \operatorname{dist}\left(x, \partial I_{T}\right)\right)^{N}} \prod_{j=1}^{3}\left(\sum_{n \in(P \cup T)_{k}} \phi_{j, k, n}^{*}(x) \psi_{j, k}^{*}(x)\left|f_{j} * \Phi_{j, k, T}(x)\right|\right)
$$

where $(P \cup T)_{k}=(P \cup T)_{l, k}$ if there exists an $l$ such that $T_{l, k} \neq \emptyset$, and $(P \cup T)_{k}=\emptyset$ if such an $l$ does not exist.

Thus $\int\left|A_{k}(x)\right| d x$ is estimated by

$$
\begin{aligned}
& \sum_{n^{\prime} \in \mathbb{Z}} \frac{C}{\left(1+2^{-k} \operatorname{dist}\left(I_{k, n^{\prime}}, \partial I_{T}\right)\right)^{N}}\left\|\prod_{j=1}^{3}\left(\sum_{n \in(P \cup T)_{k}} \phi_{j, k, n}^{*} \psi_{j, k}^{*}\left(f_{j} * \Phi_{j, k, T}\right)\right)\right\|_{L^{1}\left(I_{k, n^{\prime}}\right)} \\
& \leq \sum_{n^{\prime} \in \mathbb{Z}} \frac{C}{\left(1+2^{-k} \operatorname{dist}\left(I_{k, n^{\prime}}, \partial I_{T}\right)\right)^{N}}\left\|\sum_{n \in(P \cup T)_{k}} \phi_{1, k, n}^{*} \psi_{1, k}^{*}\left(f_{1} * \Phi_{1, k, T}\right)\right\|_{L^{\infty}\left(I_{k, n^{\prime}}\right)} \\
& \text {. }\left\|\sum_{n \in(P \cup T)_{k}} \phi_{2, k, n}^{*} \psi_{2, k}^{*}\left(f_{2} * \Phi_{2, k, T}\right)\right\|_{L^{p_{3}^{\prime}\left(I_{k, n^{\prime}}\right)}}\left\|\sum_{n \in(P \cup T)_{k}} \phi_{3, k, n}^{*} \psi_{3, k}^{*}\left(f_{3} * \Phi_{3, k, T}\right)\right\|_{L^{p_{3}\left(I_{k, n^{\prime}}\right)}}
\end{aligned}
$$

Using that $P \cup T \in S_{\mu-1}$ and Lemma 14 stated in section 4 , we obtain

$$
\begin{aligned}
& \left\|\sum_{n \in(P \cup T)_{k}} \phi_{1, k, n}^{*} \psi_{1, k}^{*}\left(f_{1} * \Phi_{1, k, T}\right)\right\|_{L^{\infty}\left(I_{k, n^{\prime}}\right)} \leq C\left\|\phi_{1, k, n^{\prime \prime}}^{*} \psi_{1, k}^{*}\left(f_{1} * \Phi_{1, k, T}\right)\right\|_{\infty} \\
& \leq C\left\|\phi_{1, k, n^{\prime \prime}}^{*} \psi_{1, k}^{*}\left(f_{1} * \Phi_{1, k, T}\right)\right\|_{p_{1}^{\prime}}^{\frac{1}{2}}\left\|\left(\phi_{1, k, n^{\prime \prime}}^{*} \psi_{1, k}^{*} e^{-2 \pi i c\left(\omega_{1, k, T}\right)(\cdot)}\left(f_{1} * \Phi_{1, k, T}\right)(\cdot)\right)^{\prime}\right\|_{p_{1}}^{\frac{1}{2}} \\
& \leq C 2^{-\eta \mu} 2^{-\frac{\mu}{p_{1}^{\prime}}}
\end{aligned}
$$

where $n^{\prime \prime} \in(P \cup T)_{k}$ which minimizes the distance to $n^{\prime}$. Since

$$
\left\|\phi_{j, k, n^{\prime \prime}}^{*} \psi_{j, k}^{*}\left(f_{j} * \Phi_{j, k, T}\right)\right\|_{p_{3}} \leq C 2^{-\frac{\mu}{p_{j}^{\prime}}}\left|I_{k, n^{\prime \prime}}\right|^{\frac{1}{p_{j}}},\left\|\phi_{j, k, n^{\prime \prime}}^{*} \psi_{j, k}^{*}\left(f_{j} * \Phi_{j, k, T}\right)\right\|_{\infty} \leq C
$$

by (4.23), interpolation gives

$$
\begin{aligned}
& \left\|\sum_{n \in(P \cup T)_{k}} \phi_{2, k, n}^{*} \psi_{2, k}^{*}\left(f_{2} * \Phi_{2, k, T}\right)\right\|_{L^{p_{3}^{\prime}\left(I_{k, n^{\prime}}\right)}} \\
& \leq C\left\|\phi_{2, k, n^{\prime \prime}}^{*} \psi_{2, k}^{*}\left(f_{2} * \Phi_{2, k, T}\right)\right\|_{p_{3}^{\prime}} \leq C 2^{-\eta \mu^{-}} 2^{-\frac{\mu}{p_{2}^{\prime}} \frac{p_{2}}{p_{3}^{\prime}}}\left|I_{k, n^{\prime \prime}}\right|^{\frac{1}{p_{3}^{\prime}}} .
\end{aligned}
$$


We also have

$$
\begin{aligned}
& \left\|\sum_{n \in(P \cup T)_{k}} \phi_{3, k, n}^{*} \psi_{3, k}^{*}\left(f_{3} * \Phi_{3, k, T}\right)\right\|_{L^{p_{3}\left(I_{k, n^{\prime}}\right)}} \\
& \quad \leq C\left\|\phi_{3, k, n^{\prime \prime}}^{*} \psi_{3, k}^{*}\left(f_{3} * \Phi_{3, k, T}\right)\right\|_{p_{3}} \leq C 2^{-\eta \mu} 2^{-\frac{\mu}{p_{3}^{\prime}}}\left|I_{k, n^{\prime \prime}}\right|^{\frac{1}{p_{3}}} .
\end{aligned}
$$

Hence we obtain

$$
\begin{aligned}
\mid \Lambda_{E, T \cup P} & \left(f_{1}, f_{2}, f_{3}\right)-\Lambda_{E, P}\left(f_{1}, f_{2}, f_{3}\right)|\leq| \Lambda_{E, T}\left(f_{1}, f_{2}, f_{3}\right)\left|+\sum_{k \leq k_{T}} \int\right| A_{k}(x) \mid d x \\
& \leq\left|\Lambda_{E, T}\left(f_{1}, f_{2}, f_{3}\right)\right|+\sum_{k \leq k_{T}} \sum_{n^{\prime} \in \mathbb{Z}} \frac{C 2^{-\eta \mu} 2^{-\left(\frac{1}{p_{1}^{\prime}}+\frac{1}{p_{2}^{\prime}} \frac{p_{2}}{p_{3}^{\prime}}+\frac{1}{p_{3}^{\prime}}\right) \mu} 2^{k}}{\left(1+2^{-k} \operatorname{dist}\left(I_{k, n^{\prime}}, \partial I_{T}\right)\right)^{N}} \\
& \leq\left|\Lambda_{E, T}\left(f_{1}, f_{2}, f_{3}\right)\right|+C 2^{-\eta \mu} 2^{-\left(\frac{1}{p_{1}^{\prime}}+\frac{1}{p_{2}^{\prime}} \frac{p_{2}}{p_{3}^{\prime}}+\frac{1}{p_{3}^{\prime}}\right) \mu}\left|I_{T}\right| .
\end{aligned}
$$

Having established Lemma 6 we can now finish the proof of estimate (1.7). Assuming Lemma 4 and Lemma 5 we obtain

$$
\begin{aligned}
\left|\Lambda_{E, S}\left(f_{1}, f_{2}, f_{3}\right)\right| \leq & \sum_{(i, j, \nu) \in H} \sum_{\mu \geq 0} \sum_{l}\left(\left|\Lambda_{E, T_{\mu, i, j, l}^{\nu}}\left(f_{1}, f_{2}, f_{3}\right)\right|+\left|\Lambda_{E, \widetilde{T}_{\mu, i, j, l}^{\nu}}\left(f_{1}, f_{2}, f_{3}\right)\right|\right) \\
& +C \sum_{(i, j, \nu) \in H} \sum_{\mu \geq 0} 2^{-\eta \mu} 2^{-\left(\frac{1}{p_{1}^{\prime}}+\frac{1}{p_{2}^{\prime}} \frac{p_{2}}{p_{3}^{\prime}}+\frac{1}{p_{3}^{\prime}}\right) \mu} \sum_{l \geq 0}\left|I_{T_{\mu, i, j, l}^{\nu}}\right| \\
\leq & C \sum_{(1, j, \nu) \in H} \sum_{\mu \geq 0} 2^{-\eta \mu} 2^{-\left(\frac{1}{p_{1}^{\prime}}+\frac{1}{p_{2}^{\prime}} \frac{p_{2}}{p_{3}^{\prime}}+\frac{1}{p_{3}^{\prime}}\right) \mu} \sum_{l \geq 0}\left|I_{T_{\mu, i, j, l}^{\nu}}\right| \\
& +C_{q_{1}} \sum_{(1, j, \nu) \in H} \sum_{\mu \geq 0} 2^{-\left(\frac{1}{p_{1}^{\prime}}+\frac{1}{p_{2}^{\prime}} \frac{p_{2}}{q_{2}}+\frac{1}{p_{3}^{\prime}} \frac{p_{3}}{q_{3}}\right) \mu} \sum_{l \geq 0}\left|I_{T_{\mu, i, j, l}^{\nu}}\right| \\
& +C_{q_{1}} \sum_{\substack{(i, j, \nu) \in H \\
i \neq 1}} \sum_{\mu \geq 0} 2^{-\left(\frac{1}{p_{1}^{\prime}}+\frac{1}{p_{2}^{\prime}} \frac{p_{2}}{q_{2}}+\frac{1}{p_{3}^{\prime}} \frac{p_{3}}{q_{3}}\right) \mu} \sum_{l}\left|I_{T_{\mu, i, j, l}^{\nu}}\right| \\
& +\sum_{\substack{(i, j, \nu) \in H \\
i \neq 1}} \sum_{\mu \geq 0} 2^{-\eta \mu} 2^{-\left(\frac{1}{p_{1}^{\prime}}+\frac{1}{p_{2}^{\prime}} \frac{p_{2}^{2}}{p_{3}^{\prime}}+\frac{1}{p_{3}^{\prime}}\right) \mu} \sum_{l \geq 0}\left|I_{T_{\mu, i, j, l}^{\nu}}\right| \\
\leq & \sum_{(i, j, \nu) \in H} \sum_{\mu \geq 0} 2^{-\left(\frac{1}{p_{1}^{\prime}}+\frac{1}{p_{2}^{\prime}} \frac{p_{2}}{p_{3}^{\prime}}+\frac{1}{p_{3}^{\prime}}\right) \mu} 2^{10 \eta p_{j}^{\prime} \mu} 2^{\mu} \\
& +C_{q_{1}} \sum_{\substack{(i, j, \nu) \in H \\
\leq}} \sum_{p_{1}, p_{2}, p_{3} .} 2^{-\left(\frac{1}{p_{1}^{\prime}}+\frac{1}{p_{2}^{\prime}} \frac{p_{2}}{q_{2}}+\frac{1}{p_{3}^{\prime}} \frac{p_{3}}{q_{3}}\right) \mu} 2^{10 \eta p_{j}^{\prime} \mu 2^{\mu}}
\end{aligned}
$$

Hence, it remains to prove Lemma 4 and Lemma 5 . This will be achieved in the following sections. 


\section{Some preliminary facts}

In this section we prove a variety of technical lemmata that will be used in the proof of Lemma 4 and Lemma 5 presented in the next sections. One important fact from these lemmata is that we have the appropriate size estimate for the trees in $S_{-1}$. We begin with the following.

Lemma 7. For any $(k, n, l) \in S$ we have the following:

$$
\begin{gathered}
\left\|\phi_{1, k, n}^{*}\left(f_{1} * \Phi_{1, k, l}\right)\right\|_{p_{1}} \leq C \inf _{x \in I_{k, n}} M_{p_{1}} f_{1}(x)\left|I_{k, n}\right|^{\frac{1}{p_{1}}} \\
\left\|\phi_{1, k, n}^{*}\left(e^{-2 \pi i c\left(\omega_{1, k, l}\right)(\cdot)}\left(f_{1} * \Phi_{1, k, l}\right)(\cdot)\right)^{\prime}\right\|_{p_{1}} \leq C \inf _{x \in I_{k, n}} M_{p_{1}} f_{1}(x)\left|I_{k, n}\right|^{\frac{1}{p_{1}}-1} \\
\left\|\phi_{1, k, n}^{*} \psi_{1, k}^{*}\left(f_{1} * \Phi_{1, k, l}\right)\right\|_{p_{1}} \leq C\left|I_{k, n}\right|^{\frac{1}{p_{1}}} \\
\left\|\phi_{1, k, n}^{*} \psi_{1, k}^{*}\left(e^{-2 \pi i c\left(\omega_{1, k, l}\right)(\cdot)}\left(f_{1} * \Phi_{1, k, l}\right)(\cdot)\right)^{\prime}\right\|_{p_{1}} \leq C\left|I_{k, n}\right|^{\frac{1}{p_{1}}-1} \cdot
\end{gathered}
$$

Proof. Since $\phi_{1, k, n}^{*}(x) \leq C\left(1+2^{-k} \operatorname{dist}\left(x, I_{k, n}\right)\right)^{-N}$ we obtain

$$
\left\|\phi_{1, k, n}^{*}\left(f_{1} * \Phi_{1, k, l}\right)\right\|_{p_{1}}^{p_{1}} \leq C\left(\inf _{x \in I_{k, n}} M_{p_{1}} f_{1}(x)\right)^{p_{1}}\left|I_{k, n}\right| .
$$

This proves $(4.1)$. Note that $\left(e^{-2 \pi i c\left(\omega_{1, k, l}\right)(\cdot)}\left(f_{1} * \Phi_{1, k, l}\right)(\cdot)\right)^{\prime}(x)$ is equal to

$$
\int f_{1}(y) e^{-2 \pi i c\left(\omega_{1, k, l}\right) y}\left(\Phi_{1, k, l}(\cdot) e^{-2 \pi i c\left(\omega_{1, k, l}\right)(\cdot)}\right)^{\prime}(x-y) d y
$$

and

$$
\left|\left(\Phi_{1, k, l}(\cdot) e^{-2 \pi i c\left(\omega_{1, k, l}\right)(\cdot)}\right)^{\prime}(x)\right| \leq \frac{C 2^{-2 k}}{\left(1+2^{-k}|x|\right)^{N}}
$$

Using this estimate and a similar argument as before we obtain (4.2).

We now prove (4.3). We may assume that $I_{k, n} \subset E$, otherwise (4.3) follows immediately from (4.1). Pick a number $A \geq 1$ such that $A I_{k, n} \subset E$ and $2 A I_{k, n} \cap E^{c} \neq \emptyset$. Then by $\psi_{1, k}^{*}(x) \leq\left(1+2^{-k} \operatorname{dist}\left(x, E^{c}\right)\right)^{-2 N}$, we have

$$
\begin{aligned}
\| \phi_{1, k, n}^{*} \psi_{1, k}^{*}\left(f_{1}\right. & \left.* \Phi_{1, k, l}\right) \|_{p_{1}}^{p_{1}} \\
& \leq C A^{-N} \int \frac{1}{\left(1+2^{-k} \operatorname{dist}\left(x, I_{k, n}\right)\right)^{N}} \int \frac{2^{-k}\left|f_{1}(y)\right|^{p_{1}}}{\left(1+2^{-k}|x-y|\right)^{N}} d y d x \\
& \leq C A^{-N}\left(\inf _{x \in I_{k, n}} M_{p_{1}} f_{1}(x)\right)^{p_{1}}\left|I_{k, n}\right| \leq C\left|I_{k, n}\right|,
\end{aligned}
$$

using the fact that the maximal function is an $A_{1}$ weight. This completes the proof of (4.3). The proof of (4.4) is similar. 
Next we have the following.

Lemma 8. For any tree $T$ of type 1 and any $j \in\{2,3\}$ we have

$$
\begin{gathered}
\left\|\left(\sum_{(k, n) \in T}\left|\phi_{j, k, n}^{*}\left(f_{j} * \Phi_{j, k, T}\right)\right|^{2}\right)^{\frac{1}{2}}\right\|_{p_{j}} \leq C \inf _{x \in I_{T}} M_{p_{j}} f_{j}(x)\left|I_{T}\right|^{\frac{1}{p_{j}}}, \\
\left\|\left(\sum_{(k, n) \in T}\left|\phi_{j, k, n}^{*} \psi_{j, k}^{*}\left(f_{j} * \Phi_{j, k, T}\right)\right|^{2}\right)^{\frac{1}{2}}\right\|_{p_{j}} \leq C\left|I_{T}\right|^{\frac{1}{p_{j}}} .
\end{gathered}
$$

Proof. First, we prove that for any $f \in \mathcal{S}$,

$$
\left\|\left(\sum_{(k, n) \in T}\left|\phi_{j, k, n}^{*}\left(f * \Phi_{j, k, T}\right)\right|^{2}\right)^{\frac{1}{2}}\right\|_{p_{j}} \leq C\|f\|_{p_{j}}
$$

Observe that $f * \Phi_{j, k, T}(x)=g_{k} * \Phi_{j, k, T}(x)$, where $g_{k}=\left(\widehat{f} 1_{\omega_{j, k, T}}\right)^{\vee}$, and $\left\{\omega_{j, k, T}\right\}_{k}$ is a lacunary family of disjoint intervals. Therefore, by the Fefferman-Stein maximal inequality [7] and by the Littlewood-Paley theorem, we have

$$
\begin{array}{r}
\left\|\left(\sum_{(k, n) \in T}\left|\phi_{j, k, n}^{*}\left(f * \Phi_{j, k, T}\right)\right|^{2}\right)^{\frac{1}{2}}\right\|_{p_{j}} \leq\left\|\left(\sum_{k}\left|g_{k} * \Phi_{j, k, T}\right|^{2}\right)^{\frac{1}{2}}\right\|_{p_{j}} \\
\leq\left\|\left(\sum_{k}\left|M g_{k}\right|^{2}\right)^{\frac{1}{2}}\right\|_{p_{j}} \leq C\left\|\left(\sum_{k}\left|g_{k}\right|^{2}\right)^{\frac{1}{2}}\right\|_{p_{j}} \leq C\|f\|_{p_{j}} .
\end{array}
$$

This is (4.7).

Now we begin by proving (4.5). The sum

$$
\left\|\left(\sum_{(k, n) \in T}\left|\phi_{j, k, n}^{*}\left(f_{j} * \Phi_{j, k, T}\right)\right|^{2}\right)^{\frac{1}{2}}\right\|_{p_{j}}
$$

is estimated by two times the expression

$$
\left\|\left(\sum_{(k, n) \in T}\left|\phi_{j, k, n}^{*}\left(\left(f_{j} 1_{2 I_{T}}\right) * \Phi_{j, k, T}\right)\right|^{2}\right)^{\frac{1}{2}}\right\|_{p_{j}}+\left\|\left(\sum_{(k, n) \in T}\left|\phi_{j, k, n}^{*}\left(\left(f_{j} 1_{\left(2 I_{T}\right)}\right) * \Phi_{j, k, T}\right)\right|^{2}\right)^{\frac{1}{2}}\right\|_{p_{j}} .
$$

Using (4.7), we can estimate the first term above by

$$
\left\|\left(\sum_{(k, n) \in T}\left|\phi_{j, k, n}^{*}\left(\left(f_{j} 1_{2 I_{T}}\right) * \Phi_{j, k, T}\right)\right|^{2}\right)^{\frac{1}{2}}\right\|_{p_{j}} \leq C\left\|f_{j} 1_{2 I_{T}}\right\|_{p_{j}} \leq C \inf _{x \in I_{T}} M_{p_{j}} f_{j}(x)\left|I_{T}\right|^{\frac{1}{p_{j}}}
$$


But $\left|\phi_{j, k, m}^{*}(x)\right| \leq C\left(1+2^{-k+m} \operatorname{dist}\left(x, I_{k, n}\right)\right)^{-N}$ and using (2.7) or (2.14) we obtain

$$
\begin{gathered}
\left\|\left(\sum_{(k, n) \in T}\left|\phi_{j, k, n}^{*}\left(\left(f_{j} 1_{\left(2 I_{T}\right)^{c}}\right) * \Phi_{j, k, T}\right)\right|^{2}\right)^{\frac{1}{2}}\right\|_{p_{j}}^{p_{j}} \\
\leq C \sum_{(k, n) \in T} \frac{\left|I_{k, n}\right|}{\left(1+2^{-k} \operatorname{dist}\left(\left(2 I_{T}\right)^{c}, I_{k, n}\right)\right)^{N}}\left(\inf _{x \in I_{T}} M_{p_{j}} f_{j}(x)\right)^{p_{j}} \\
\leq C\left(\inf _{x \in I_{T}} M_{p_{j}} f_{j}(x)\right)^{p_{j}}\left|I_{T}\right|,
\end{gathered}
$$

which completes the proof of (4.5).

We now turn our attention to the proof of (4.6). Assume $I_{T} \subset E$, otherwise using (4.5) we obtain (4.6) immediately. Pick $A \geq 1$ such that $A I_{T} \subset E$ and $2 A I_{T} \bigcap E^{c} \neq \emptyset$. Then since

$$
\left|\psi_{j, k}^{*}(x)\right| \leq \frac{C}{\left(1+2^{-k+m} \operatorname{dist}\left(x, E^{c}\right)\right)^{N}},
$$

we have

$$
\begin{aligned}
& \left\|\left(\sum_{(k, n) \in T}\left|\phi_{j, k, n}^{*} \psi_{j, k}^{*}\left(f_{j} * \Phi_{j, k, T}\right)\right|^{2}\right)^{\frac{1}{2}}\right\|_{p_{j}} \\
& \leq\left\|\left(\sum_{(k, n) \in T} \frac{1}{\left(1+2^{-k+m} \operatorname{dist}\left(I_{k, n}, E^{c}\right)\right)^{N}}\left|\phi_{j, k, n}^{*}\left(f_{j} * \Phi_{j, k, T}\right)\right|^{2}\right)^{\frac{1}{2}}\right\|_{p_{j}} \\
& \leq C A^{-N}\left\|\left(\sum_{(k, n) \in T}\left|\phi_{j, k, n}^{*}\left(f_{j} * \Phi_{j, k, T}\right)\right|^{2}\right)^{\frac{1}{2}}\right\| \|_{p_{j}} \\
& \leq C A^{-N} \inf _{x \in I_{T}} M_{p_{j}} f_{j}(x)\left|I_{T}\right|^{\frac{1}{p_{j}}} \leq C \inf _{x \in 2 A I_{T}} M_{p_{j}} f_{j}(x)\left|I_{T}\right|^{\frac{1}{p_{j}}} \leq C\left|I_{T}\right|^{\frac{1}{p_{j}}},
\end{aligned}
$$

where we used (4.5) in the penultimate inequality above. This proves (4.6) and thus completes the proof of this lemma.

Similarly we obtain the following lemma whose proof we omit.

Lemma 9. For any tree $T$ of type $j, j \in\{2,3\}$ we have

$$
\begin{gathered}
\left\|\left(\sum_{(k, n) \in T}\left|\phi_{1, k, n}^{*}\left(f_{1} * \Phi_{1, k, T}\right)\right|^{2}\right)^{\frac{1}{2}}\right\|_{p_{j}} \leq C \inf _{x \in I_{T}} M_{p_{1}} f_{1}(x)\left|I_{T}\right|^{\frac{1}{p_{1}}}, \\
\left\|\left(\sum_{(k, n) \in T}\left|\phi_{1, k, n}^{*} \psi_{1, k}^{*}\left(f_{1} * \Phi_{1, k, T}\right)\right|^{2}\right)^{\frac{1}{2}}\right\|_{p_{1}} \leq C\left|I_{T}\right|^{\frac{1}{p_{1}}} .
\end{gathered}
$$


Next we have the following.

Lemma 10. For $(k, n, l) \in S, \tilde{k} \in\{-L, 0, L, 2 L, 3 L, 4 L\}$, and $j \in\{2,3\}$ we have

$$
\begin{gathered}
\left\|\phi_{j, k+\tilde{k}, n}^{*}\left(f_{j} * \Phi_{j, k+\tilde{k}, l}\right)\right\|_{p_{j}} \leq C \inf _{x \in I_{k, n}} M_{p_{j}} f_{j}(x)\left|I_{k, n}\right|^{\frac{1}{p_{j}}} \\
\left\|\phi_{1, k, n}^{*}\left(f_{j} * \Phi_{j, k+m+\tilde{k}, l}\right)\right\|_{p_{j}} \leq C \inf _{x \in I_{k, n}} M_{p_{j}} f_{j}(x)\left|I_{k, n}\right|^{\frac{1}{p_{j}}} \\
\left\|\phi_{1, k, n}^{*}\left(e^{-2 \pi i c\left(\omega_{j, k+m+\tilde{k}, l}\right)(\cdot)}\left(f_{j} * \Phi_{j, k+m+\tilde{k}, l}\right)(\cdot)\right)^{\prime}\right\|_{p_{j}} \\
\leq C \inf _{x \in I_{k, n}} M_{p_{j}} f_{j}(x)\left|I_{k, n}\right|^{\frac{1}{p_{j}}-1} \\
\left\|\phi_{j, k+\tilde{k}, n}^{*} \psi_{j, k+\tilde{k}}^{*}\left(f_{j} * \Phi_{j, k+\tilde{k}, l}\right)\right\|_{p_{j}} \leq C\left|I_{k, n}\right|^{\frac{1}{p_{j}}} \\
\left\|\phi_{1, k, n}^{*} \psi_{2, k+m+\tilde{k}}^{*}\left(f_{j} * \Phi_{j, k+m+\tilde{k}, l}\right)\right\|_{p_{j}} \leq C\left|I_{k, n}\right|^{\frac{1}{p_{j}}}, \\
\left\|\phi_{1, k, n}^{*} \psi_{2, k+m+\tilde{k}}^{*}\left(e^{-2 \pi i c\left(\omega_{j, k+m+\tilde{k}, l}\right)(\cdot)}\left(f_{j} * \Phi_{j, k+m+\tilde{k}, l}\right)(\cdot)\right)^{\prime}\right\|_{p_{j}} \leq C\left|I_{k, n}\right|^{\frac{1}{p_{j}}-1} .
\end{gathered}
$$

Proof. Note

$$
\phi_{j, k+\tilde{k}, n}^{*}(x) \leq C\left(1+2^{-k+m} \operatorname{dist}\left(x, I_{k, n}\right)\right)^{-N}
$$

we have

$$
\begin{aligned}
& \left\|\phi_{j, k+\tilde{k}, n}^{*}\left(f_{j} * \Phi_{j, k+\tilde{k}, l}\right)\right\|_{p_{j}}^{p_{j}} \\
& \quad \leq C \int \frac{1}{\left(1+2^{-k+m} \operatorname{dist}\left(x, I_{k, n}\right)\right)^{N}} \int \frac{2^{-k+m}\left|f_{j}(y)\right|^{p_{j}}}{\left(1+2^{-k+m}|x-y|\right)^{N}} d y d x \\
& \quad \leq C \int \frac{\left|f_{j}(y)\right|^{p_{j}}}{\left(1+2^{-k+m} \operatorname{dist}\left(y, I_{k, n}\right)\right)^{N}} d y \leq C\left(\inf _{x \in I_{k, n}} M_{p_{j}} f_{j}(x)\right)^{p_{j}}\left|I_{k, n}\right| .
\end{aligned}
$$

This proves (4.10). Now we prove (4.13). Assume $I_{k, n} \subset E$, otherwise by (4.10) we have (4.13) immediately. Pick a number $A \geq 1$ such that $A I_{k, n} \subset E$ and $2 A I_{k, n} \cap E^{c} \neq \emptyset$. Then we have

$$
\left\|\phi_{j, k+\tilde{k}, n}^{*} \psi_{j, k+\tilde{k}}^{*}\left(f_{j} * \Phi_{j, k+\tilde{k}, l}\right)\right\|_{p_{j}}^{p_{j}} \leq C A^{-N}\left(\inf _{x \in I_{k, n}} M_{p_{j}} f_{j}(x)\right)^{p_{j}}\left|I_{k, n}\right| \leq C\left|I_{k, n}\right| .
$$

This completes the proof of (4.13). Similarly, we obtain (4.11), (4.12), (4.14) and (4.15). 
Lemma 11. For a convex tree $T$ of type $j, j \in\{2,3\}$ we have

$$
\begin{aligned}
& \left\|\left(\sum_{(k, n) \in T}\left|\phi_{j, k, n}^{*}\left(f_{j} * \theta_{j, k, T}^{+}\right)\right|^{2}\right)^{\frac{1}{2}}\right\|_{p_{j}} \leq C \inf _{x \in I_{T}} M_{p_{j}} f_{j}(x)\left|I_{T}\right|^{\frac{1}{p_{j}}}, \\
& \left\|\left(\sum_{(k, n) \in T}\left|\phi_{j, k, n}^{*}\left(f_{j} * \theta_{j, k, T}^{-}\right)\right|^{2}\right)^{\frac{1}{2}}\right\|_{p_{j}} \leq C \inf _{x \in I_{T}} M_{p_{j}} f_{j}(x)\left|I_{T}\right|^{\frac{1}{p_{j}}}, \\
& \left\|\left(\sum_{(k, n) \in T}\left|\phi_{j, k, n}^{*} \psi_{j, k}^{*}\left(f_{j} * \theta_{j, k, T}^{+}\right)\right|^{2}\right)^{\frac{1}{2}}\right\|_{p_{j}} \leq C\left|I_{T}\right|^{\frac{1}{p_{j}}}, \\
& \left\|\left(\sum_{(k, n) \in T}\left|\phi_{j, k, n}^{*} \psi_{j, k}^{*}\left(f_{j} * \theta_{j, k, T}^{-}\right)\right|^{2}\right)^{\frac{1}{2}}\right\|_{p_{j}} \leq C\left|I_{T}\right|^{\frac{1}{p_{j}}} .
\end{aligned}
$$

Proof. This lemma is similar to Lemma 8 and we omit its proof.

Lemma 12. For $\tilde{k} \in\{-L, 0, L, 2 L, 3 L, 4 L\}$, let $T$ be a tree of type $j$, $j \in\{2,3\}$

$$
\begin{gathered}
\left\|\left(\sum_{k} \sum_{J \in \Delta_{k-m, T}}\left|\rho_{k-m, J}\left(f_{j} * \Phi_{j, k+\tilde{k}, T}\right)\right|^{2}\right)^{\frac{1}{2}}\right\|_{p_{j}} \leq C \inf _{x \in I_{T}} M_{p_{j}} f_{j}(x)\left|I_{T}\right|^{\frac{1}{p_{j}}}, \\
\left\|\left(\sum_{k} \sum_{J \in \Delta_{k-m, T}}\left|\rho_{k-m, J}\left(f_{j} * \Phi_{j, k+\tilde{k}, T}\right)\right|^{2}\right)^{\frac{1}{2}}\right\|_{p_{j}} \leq C\left|I_{T}\right|^{\frac{1}{p_{j}}} .
\end{gathered}
$$

Proof. We prove (4.21) first. Since $p_{j}<2$, we have

$$
\begin{aligned}
& \left\|\left(\sum_{k} \sum_{J \in \Delta_{k-m, T}}\left|\rho_{k-m, J}\left(f_{j} * \Phi_{j, k+\tilde{k}, T}\right)\right|^{2}\right)^{\frac{1}{2}}\right\|_{p_{j}} \\
& \quad \leq C\left\|\left(\sum_{k} \sum_{J \in \Delta_{k-m, T}} \frac{\left(\inf _{x \in J} M f_{j}(x)\right)^{2}}{\left(1+2^{-k+m} \operatorname{dist}(x, J)\right)^{N}}\right)^{\frac{1}{2}}\right\|_{p_{j}} \\
& \quad \leq C\left(\sum_{k} \sum_{J \in \Delta_{k-m, T}}\left(\inf _{x \in 8 J} M_{p_{j}} f_{j}(x)\right)^{p_{j}}|J|\right)^{\frac{1}{p_{j}}} \quad\left(\text { since } 8 J \cap E^{c} \neq \emptyset\right) \\
& \quad \leq C\left(\sum_{k} \sum_{J \in \Delta_{k-m, T}}|J|\right)^{\frac{1}{p_{j}}} \leq C\left|I_{T}\right|^{\frac{1}{p_{j}}}
\end{aligned}
$$

because the union of $\Delta_{k-m}$ is a set of pairwise disjoint intervals. 
On the other hand, note that

$$
\begin{array}{r}
\left(\sum_{k} \sum_{J \in \Delta_{k-m, T}}\left(\inf _{x \in J} M f_{j}(x)\right)^{p_{j}}|J|\right)^{\frac{1}{p_{j}}} \leq\left(\sum_{k} \sum_{J \in \Delta_{k-m, T}} \int_{J}\left(M f_{j}(x)\right)^{p_{j}} d x\right)^{\frac{1}{p_{j}}} \\
\leq\left(\int \underset{J \in \bigcup_{k} \Delta_{k-m, T} J}{ }\left(M f_{j}(x)\right)^{p_{j}} d x\right)^{\frac{1}{p_{j}}} \leq C\left\|f_{j}\right\|_{p_{j}}
\end{array}
$$

Hence, we obtain that for $f \in \mathcal{S}$ we have the inequality

$$
\left\|\left(\sum_{k} \sum_{J \in \Delta_{k-m, T}}\left|\rho_{k-m, J}\left(f * \Phi_{j, k+\tilde{k}, T}\right)\right|^{2}\right)^{\frac{1}{2}}\right\|_{p_{j}} \leq C\|f\|_{p_{j}} .
$$

We now prove (4.20). We have

$$
\left\|\left(\sum_{k} \sum_{J \in \Delta_{k-m, T}}\left|\rho_{k-m, J}\left(f_{j} * \Phi_{j, k+\tilde{k}, T}\right)\right|^{2}\right)^{\frac{1}{2}}\right\|_{p_{j}} \leq D_{1}+D_{2},
$$

where

$$
\begin{aligned}
& D_{1}=\left\|\left(\sum_{k} \sum_{J \in \Delta_{k-m, T}}\left|\rho_{k-m, J}\left(\left(f_{j} 1_{2 I_{T}}\right) * \Phi_{j, k+\tilde{k}, T}\right)\right|^{2}\right)^{\frac{1}{2}}\right\|_{p_{j}} \\
& D_{2}=\left\|\left(\sum_{k} \sum_{J \in \Delta_{k-m, T}}\left|\rho_{k-m, J}\left(\left(f_{j} 1_{\left(2 I_{T}\right)^{c}}\right) * \Phi_{j, k+\tilde{k}, T}\right)\right|^{2}\right)^{\frac{1}{2}}\right\|_{p_{j}} .
\end{aligned}
$$

By (4.22) it is easy to see that

$$
D_{1} \leq C\left\|f_{j} 1_{2 I_{T}}\right\|_{p_{j}} \leq C\left|I_{T}\right|^{\frac{1}{p_{j}}} \inf _{x \in I_{T}} M_{p_{j}} f_{j}(x) .
$$

For $D_{2}$, we have

$$
\begin{aligned}
& D_{2}^{p_{j}} \leq \\
& \leq \int\left(\sum_{k} \sum_{J \in \Delta_{k-m, T}} \frac{C\left(\int_{\left(2 I_{T}\right)^{c}}\left|f_{j}(y)\right| 2^{-k+m}\left(1+2^{-k+m}|x-y|\right)^{-(N+2)} d y\right)^{2}}{\left(1+2^{-k+m} \operatorname{dist}(x, J)\right)^{2 N+2}}\right)^{\frac{p_{j}}{2}} d x \\
& \leq\left(\inf _{x \in I_{T}} M f_{j}(x)\right)^{p_{j}} \sum_{k} \sum_{J \in \Delta_{k-m, T}}|J| \leq\left(\inf _{x \in I_{T}} M_{p_{j}} f_{j}(x)\right)^{p_{j}}\left|I_{T}\right|,
\end{aligned}
$$

which proves (4.20) and thus completes the proof of Lemma 12. 
Lemma 13. Let $j \in\{2,3\}$ and $T \subset S$ be a convex tree of type $j$. Then we have

$$
\begin{gathered}
\left\|\psi_{j, k}^{*}\left(f_{j} * \Phi_{j, k, l}\right)\right\|_{\infty} \leq C, \\
\left\|\left(\sum_{k}\left|\sum_{n \in T_{k}} \phi_{j, k, n}^{*} \psi_{j, k}^{*}\left(f_{j} *\left(\Phi_{j, k-L, T}-\Phi_{j, k, T}\right)\right)\right|^{2}\right)^{\frac{1}{2}}\right\|_{B M O} \leq C,
\end{gathered}
$$

where $C$ is independent of $m$ and BMO denotes dyadic BMO.

Proof. (4.23) holds since $\psi_{j, k}^{*}(x) \leq C\left(1+2^{-k+m} \operatorname{dist}\left(x, E^{c}\right)\right)^{-N}$ and

$$
\left|f_{j} * \Phi_{j, k, l}(x)\right| \leq C\left(1+2^{-k+m} \operatorname{dist}\left(x, E^{c}\right)\right)^{2} .
$$

Now we prove (4.24). Let $J=\left[2^{k_{J}} n_{J}, 2^{k_{J}}\left(n_{J}+1\right)\right]$, then

$\inf _{c}\left\|\left(\sum_{k}\left|\sum_{n \in T_{k}} \phi_{j, k, n}^{*} \psi_{j, k}^{*}\left(f_{j} *\left(\Phi_{j, k-L, T}-\Phi_{j, k, T}\right)\right)\right|^{2}\right)^{\frac{1}{2}}-c\right\|_{L^{p_{j}(J)}}^{p_{j}} \leq C\left(B_{1}+B_{2}\right)$,

where

$$
\begin{gathered}
B_{1}=\inf _{c}\left\|\left(\sum_{k \geq k_{J}+m}\left|\sum_{n \in T_{k}} \phi_{j, k, n}^{*} \psi_{j, k}^{*}\left(f_{j} *\left(\Phi_{j, k-L, T}-\Phi_{j, k, T}\right)\right)\right|^{2}\right)^{\frac{1}{2}}-c\right\|_{L^{2}(J)}^{2} \\
B_{2}=\left\|\left(\sum_{k<k_{J}+m}\left|\sum_{n \in T_{k}} \phi_{j, k, n}^{*} \psi_{j, k}^{*}\left(f_{j} *\left(\Phi_{j, k-L, T}-\Phi_{j, k, T}\right)\right)\right|^{2}\right)^{\frac{1}{2}}\right\|_{L^{p_{j}(J)}}^{p_{j}} .
\end{gathered}
$$

For $B_{1}$ using (4.23), we obtain

$$
\begin{aligned}
& \inf _{c}\left\|\left(\sum_{k \geq k_{J}+m}\left|\sum_{n \in T_{k}} \phi_{j, k, n}^{*} \psi_{j, k}^{*}\left(f_{j} *\left(\Phi_{j, k-L, T}-\Phi_{j, k, T}\right)\right)\right|^{2}\right)^{\frac{1}{2}}-c\right\|_{L^{2}(J)}^{p_{j}}|J|^{1-\frac{p_{j}}{2}} \\
& \leq\left(\left.\inf _{c}\left|\sum_{k \geq k_{J}+m}\right| \sum_{n \in T_{k}} \phi_{j, k, n}^{*} \psi_{j, k}^{*}\left(f_{j} *\left(\Phi_{j, k-L, T}-\Phi_{j, k, T}\right)\right)\right|^{2}-c^{2} \|_{L^{1}(J)}\right)^{\frac{p_{j}}{2}}|J|^{1-\frac{p_{j}}{2}} \\
& \leq\left(\left.|J|^{2} \sum_{k \geq k_{J}+m}\left\|\left(\left(\sum_{n \in T_{k}} \phi_{j, k, n}^{*} \psi_{j, k}^{*} e^{-2 \pi i c\left(\omega_{j, k-L, T}\right) \cdot(\cdot)}\left(f_{j} *\left(\Phi_{j, k-L, T}-\Phi_{j, k, T}\right)\right)\right)^{2}\right)^{\prime}\right\|\right|_{\infty}\right)^{\frac{p_{j}}{2}} \\
& \cdot C|J|^{1-\frac{p_{j}}{2}} \\
& \leq C\left(|J|^{2} \sum_{k \geq k_{J}+m} 2^{-k+m}\right)^{\frac{p_{j}}{2}}|J|^{1-\frac{p_{j}}{2}} \leq C|J|^{\frac{p_{j}}{2}}|J|^{1-\frac{p_{j}}{2}} \leq C|J| .
\end{aligned}
$$


For $B_{2}$ we have

$$
\left\|\left(\sum_{k<k_{J}+m}\left|\sum_{n \in T_{k}} \phi_{j, k, n}^{*} \psi_{j, k}^{*}\left(f_{j} *\left(\Phi_{j, k-L, T}-\Phi_{j, k, T}\right)\right)\right|^{2}\right)^{\frac{1}{2}}\right\|_{L^{p_{j}(J)}}^{p_{j}} \leq C\left(B_{21}+B_{22}\right),
$$

where

$$
\begin{aligned}
& B_{21}=\left\|\left(\sum_{k<k_{J}+m}\left|\sum_{n \in T_{k}} \phi_{j, k, n}^{*} \psi_{j, k}^{*}\left(\left(f_{j} 1_{2 J}\right) *\left(\Phi_{j, k-L, T}-\Phi_{j, k, T}\right)\right)\right|^{2}\right)^{\frac{1}{2}}\right\|_{L^{p_{j}(J)}}^{p_{j}}, \\
& B_{22}=\left\|\left(\sum_{k<k_{J}+m}\left|\sum_{n \in T_{k}} \phi_{j, k, n}^{*} \psi_{j, k}^{*}\left(\left(f_{j} 1_{(2 J)^{c}}\right) *\left(\Phi_{j, k-L, T}-\Phi_{j, k, T}\right)\right)\right|^{2}\right)^{\frac{1}{2}}\right\|_{L^{p_{j}}(J)}^{p_{j}} .
\end{aligned}
$$

For $B_{21}$, as we proved (4.7), we have

$$
\begin{aligned}
B_{21} & \leq\left(\sup _{k<k_{J}+m}\left\|\psi_{j, k}^{*}\right\|_{L^{\infty}(J)}\right)\left\|f_{j} 1_{2 J}\right\|_{p_{j}}^{p_{j}} \\
& \leq \frac{C}{\left(1+2^{-k_{J}} \operatorname{dist}\left(J, E^{c}\right)\right)^{N}}\left\|f_{j} 1_{2 J}\right\|_{p_{j}}^{p_{j}} \leq C|J| .
\end{aligned}
$$

For $B_{22}$, notice that

$$
\begin{aligned}
& \left\|f_{j} 1_{(2 J)^{c}} * \Phi_{j, k, T}\right\|_{L^{\infty}(J)} \\
& \leq \frac{C}{\left(1+2^{-k+m}|J|\right)^{N}}\left\|\left(\int_{(2 J)^{c}}\left|f_{j}(y)\right|^{p_{j}} \frac{2^{-k+m}}{\left(1+2^{-k+m}|\cdot-y|\right)^{N}} d y\right)^{\frac{1}{p_{j}}}\right\|_{L^{\infty}(J)} \\
& \leq \frac{C\left(1+2^{-k+m}|J|+2^{-k+m} \operatorname{dist}\left(J, E^{c}\right)\right)^{\frac{2}{p_{j}}}}{\left(1+2^{-k+m}|J|\right)^{N}} \\
& \leq C 2^{\left(k-m-k_{J}\right) N}\left(1+2^{-k+m} \operatorname{dist}\left(J, E^{c}\right)\right)^{2}
\end{aligned}
$$

and $\left\|\psi_{j, k}^{*}\right\|_{L^{\infty}(J)} \leq C\left(1+2^{-k+m} \operatorname{dist}\left(J, E^{c}\right)\right)^{-N}$. We have

$$
\begin{aligned}
B_{22} & \leq C \sum_{k<k_{J}+m} \frac{\left\|f_{j} 1_{(2 J)^{c}} * \Phi_{j, k, T}\right\|_{L^{\infty}(J)}^{p_{j}}|J|}{\left(1+2^{-k+m} \operatorname{dist}\left(J, E^{c}\right)\right)^{N}} \\
& \leq C \sum_{k<k_{J}+m} 2^{\left(k-m-k_{J}\right) p_{j} N}|J| \leq C|J| .
\end{aligned}
$$

This completes the proof of the lemma. 
Lemma 14. Let $f \in \mathcal{S},(k, n, l) \in S$ such that

$$
\begin{gathered}
\left\|\phi_{1, k, n}^{*} \psi_{1, k}^{*}\left(f * \Phi_{1, k, l}\right)\right\|_{p_{1}} \leq 2^{-\eta \mu} 2^{-\frac{\mu}{p_{1}^{\tau}}}\left|I_{k, n}\right|^{\frac{1}{p_{1}}} \\
\left\|\phi_{1, k, n}^{*} \psi_{1, k}^{*}\left(e^{-2 \pi i c\left(\omega_{1, k, l}\right)(\cdot)}\left(f * \Phi_{1, k, l}\right)(\cdot)\right)^{\prime}\right\|_{p_{1}} \leq 2^{-\eta \mu_{2}} 2^{-\frac{\mu}{p_{1}^{\prime}}}\left|I_{k, n}\right|^{\frac{1}{p_{1}}-1} .
\end{gathered}
$$

Then we have

$$
\left\|\phi_{1, k, n}^{*} \psi_{1, k}^{*} e^{-2 \pi i c\left(\omega_{1, k, l}\right)(\cdot)}\left(f * \Phi_{1, k, l}\right)\right\|_{B M O} \leq C 2^{-\eta \mu_{2}} 2^{-\frac{\mu}{p_{1}^{\prime}}} .
$$

Proof. Let $J=\left[2^{k_{J}} n_{J}, 2^{k_{J}}\left(n_{J}+1\right)\right]$. First, assume that $\left|I_{k, n}\right| \leq|J|$, then by $(4.25)$ we have

$$
\begin{aligned}
& \inf _{c} \int_{J}\left|\phi_{1, k, n}^{*}(x) \psi_{1, k}^{*}(x) e^{-2 \pi i c\left(\omega_{1, k, l}\right)(x)}\left(f * \Phi_{1, k, l}\right)(x)-c\right| d x \\
& \quad \leq \int_{J}\left|\phi_{1, k, n}^{*}(x) \psi_{1, k}^{*}(x)\left(f * \Phi_{1, k, l}\right)(x)\right| d x \\
& \quad \leq\left\|\phi_{1, k, n}^{*} \psi_{1, k}^{*}\left(f * \Phi_{1, k, l}\right)\right\|_{p_{1}}|J|^{1-\frac{1}{p_{1}}} \\
& \quad \leq 2^{-\eta \mu_{2}} 2^{-\frac{\mu}{p_{1}^{\prime}}\left|I_{k, n}\right|^{\frac{1}{p_{1}}}|J|^{1-\frac{1}{p_{1}}} \leq 2^{-\eta \mu} 2^{-\frac{\mu}{p_{1}^{\prime}}}|J| .}
\end{aligned}
$$

Now assume that $\left|I_{k, n}\right|>|J|$, then by (4.25) and (4.26) we obtain

$$
\begin{aligned}
\inf _{c} & \int_{J}\left|\phi_{1, k, n}^{*}(x) \psi_{1, k}^{*}(x) e^{-2 \pi i c\left(\omega_{1, k, l}\right)(x)}\left(f * \Phi_{1, k, l}\right)(x)-c\right| d x \\
\leq & |J| \int_{J}\left|\left(\phi_{1, k, n}^{*}(\cdot) \psi_{1, k}^{*}(\cdot) e^{-2 \pi i c\left(\omega_{1, k, l}\right)(\cdot)}\left(f * \Phi_{1, k, l}\right)(\cdot)\right)^{\prime}(x)\right| d x \\
\leq & |J| \int_{J}\left|\left(\phi_{1, k, n}^{*}(x) \psi_{1, k}^{*}\right)^{\prime}(x)\left(f * \Phi_{1, k, l}\right)(x)\right| d x \\
& +|J| \int_{J}\left|\phi_{1, k, n}^{*}(x) \psi_{1, k}^{*}(x)\left(e^{-2 \pi i c\left(\omega_{1, k, l}\right)(\cdot)}\left(f * \Phi_{1, k, l}\right)(\cdot)\right)^{\prime}(x)\right| d x \\
\leq & C|J| 2^{-k}\left\|\phi_{1, k, n}^{*} \psi_{1, k}^{*}\left(f * \Phi_{1, k, l}\right)\right\|_{p_{1}}|J|^{1-\frac{1}{p_{1}}} \\
& +|J||| \phi_{1, k, n}^{*} \psi_{1, k}^{*}\left(e^{-2 \pi i c\left(\omega_{1, k, l}\right)(\cdot)}\left(f * \Phi_{1, k, l}\right)(\cdot)\right)^{\prime} \|\left.\right|_{p_{1}}|J|^{1-\frac{1}{p_{1}}} \\
\leq & C|J| 2^{-\eta \mu} 2^{-\frac{\mu}{p_{1}^{\prime}}}\left|I_{k, n}\right|^{\frac{1}{p_{1}}-1}|J|^{1-\frac{1}{p_{1}}} \leq C \cdot 2^{-\eta \mu} 2^{-\frac{\mu}{p_{1}^{\prime}}}|J|,
\end{aligned}
$$

since $\left|\left(\phi_{1, k, n}^{*} \psi_{1, k}^{*}\right)^{\prime}\right| \leq C\left|I_{k, n}\right|^{-1}\left|\phi_{1, k, n}^{*}(x) \psi_{1, k}^{*}\right|$. Therefore we obtain (4.27). 


\section{The size estimate for the trees}

Having proved all these preliminary lemmata we now turn our attention to the proof of Lemma 4. This section is entirely devoted to the proof of this lemma. First, we prove (3.18). For a tree $T$ of type 1 and $T \subset S_{\mu}$, we have

$$
\begin{gathered}
\left|\Lambda_{E, T}\left(f_{1}, f_{2}, f_{3}\right)\right| \leq \sum_{k} \int \prod_{j=1}^{3}\left|\left(\sum_{n \in T_{k}} \phi_{j, k, n}(x) \psi_{j, k}(x)\left(f_{j} * \Phi_{j, k, T}\right)\right)\right| d x \\
\leq \int \sup _{k}\left|\sum_{n \in T_{k}} \phi_{1, k, n}(x) \psi_{1, k}(x)\left(f_{1} * \Phi_{1, k, T}\right)(x)\right| \\
\cdot \prod_{j=2}^{3}\left(\sum_{k}\left|\sum_{n \in T_{k}} \phi_{j, k, n}(x) \psi_{j, k}(x)\left(f_{j} * \Phi_{j, k, T}\right)(x)\right|^{2}\right)^{\frac{1}{2}} d x \\
\leq\left\|\sup _{k}\left|\sum_{n \in T_{k}} \phi_{1, k, n} \psi_{1, k}\left(f_{1} * \Phi_{1, k, T}\right)\right||| \mid\left(\sum_{k}\left|\sum_{n \in T_{k}} \phi_{2, k, n} \psi_{2, k}\left(f_{2} * \Phi_{2, k, T}\right)\right|^{2}\right)^{\frac{1}{2}}\right\|_{p_{3}^{\prime}} \\
\cdot\left\|\left(\sum_{k}\left|\sum_{n \in T_{k}} \phi_{3, k, n} \psi_{3, k}\left(f_{3} * \Phi_{3, k, T}\right)\right|^{2}\right)^{\frac{1}{2}}\right\|_{p_{3}} .
\end{gathered}
$$

Observe that

$$
\begin{aligned}
& \left\|\sup _{k}\left|\sum_{n \in T_{k}} \phi_{1, k, n} \psi_{1, k}\left(f_{1} * \Phi_{1, k, T}\right)\right|\right\|_{\infty} \leq \sup _{(k, n) \in T}\left\|\phi_{1, k, n}^{*} \psi_{1, k}^{*}\left(f_{1} * \Phi_{1, k, T}\right)\right\|_{\infty} \\
& \leq \sup _{(k, n) \in T}\left\|\phi_{1, k, n}^{*} \psi_{1, k}^{*}\left(f_{1} * \Phi_{1, k, T}\right)\right\|_{p_{1}^{\prime}}^{\frac{1}{2}}\left\|\left(\phi_{1, k, n}^{*} \psi_{1, k}^{*} e^{-2 \pi i c\left(\omega_{1, k, T}\right)(\cdot)}\left(f_{1} * \Phi_{1, k, T}\right)(\cdot)\right)^{\prime}\right\|_{p_{1}}^{\frac{1}{2}} \\
& \leq C 2^{-\eta \mu} 2^{-\frac{\mu}{p_{1}^{\prime}}}
\end{aligned}
$$

here we use Lemma 14 and interpolation for the first factor.

Since $T$ is a tree of type 1 , as in the proof of Lemma 13, we obtain

$$
\left\|\left(\sum_{k}\left|\sum_{n \in T_{k}} \phi_{2, k, n} \psi_{2, k}\left(f_{2} * \Phi_{2, k, T}\right)\right|^{2}\right)^{\frac{1}{2}}\right\|_{B M O} \leq C .
$$

Notice that since $T \in S_{\mu}$, we have for $j \in\{2,3\}$

$$
\left\|\left(\sum_{k}\left|\sum_{n \in T_{k}} \phi_{j, k, n} \psi_{j, k}\left(f_{j} * \Phi_{j, k, T}\right)\right|^{2}\right)^{\frac{1}{2}}\right\|_{p_{j}} \leq C 2^{-\frac{\mu}{p_{j}^{\prime}}}\left|I_{T}\right|^{\frac{1}{p_{j}}} .
$$

Hence, by interpolation we have

$$
\left\|\left(\sum_{k}\left|\sum_{n \in T_{k}} \phi_{2, k, n} \psi_{2, k}\left(f_{2} * \Phi_{2, k, T}\right)\right|^{2}\right)^{\frac{1}{2}}\right\|_{p_{3}^{\prime}} \leq C 2^{-\frac{\mu}{p_{2}^{\prime}} \frac{p_{2}}{p_{3}^{\prime}}}\left|I_{T}\right|^{\frac{1}{p_{3}^{\prime}}} .
$$


Thus we have

$$
\left|\Lambda_{E, T}\left(f_{1}, f_{2}, f_{3}\right)\right| \leq C 2^{-\eta \mu} 2^{-\left(\frac{1}{p_{1}^{\prime}}+\frac{1}{p_{2}^{\prime}} \frac{p_{2}}{p_{3}^{\prime}}+\frac{1}{p_{3}^{\prime}}\right) \mu}
$$

This completes the proof of (3.18) for trees of type 1. We now turn our attention to the proof of (3.19). Let

$$
f_{i, k}(x)=\sum_{n \in T_{k}} \phi_{i, k, n}(x) \psi_{i, k}(x)\left(f_{i} * \Phi_{i, k, T}\right)(x),
$$

for $i=1,2,3$. Then $\sum_{k \in \mathbb{Z}_{r}} f_{1, k} f_{2, k} f_{3, k}$ is equal to

$$
\sum_{k \in \mathbb{Z}_{r}} f_{1, k} f_{2, k+m+L} f_{3, k+m+L}+\sum_{\substack{k \in \mathbb{Z}_{r} \\ 0 \leq \tilde{k} \leq m}} \sum_{\substack{\tilde{k} \in \mathbb{Z}_{0} \\ 0 \leq k}} f_{1, k}\left(f_{2, k+\tilde{k}} f_{3, k+\tilde{k}}-f_{2, k+\tilde{k}+L} f_{3, k+\tilde{k}+L}\right) .
$$

Note that $-\operatorname{supp} \widehat{f}_{3, k+m+L}<\operatorname{supp} \widehat{f}_{1, k}+\operatorname{supp} \widehat{f}_{2, k+m+L}$, which is proved in [8]. Thus, we have that the integral of the first term in (5.4) is zero. Thus it is sufficient to consider the second term in (5.4). As in [8], we write the second term in (5.4) as

$$
\sum_{k \in \mathbb{Z}_{r}}\left(\sum_{\substack{\tilde{k} \in \mathbb{Z}_{0} \\ 0 \leq \hat{k} \leq m}} f_{1, k-\tilde{k}}\right)\left(f_{2, k} f_{3, k}-f_{2, k+L} f_{3, k+L}\right)=I_{1}+I_{2}+I_{3}+I_{4}+I_{5},
$$

where

$$
\begin{aligned}
& I_{1}=\sum_{k \in \mathbb{Z}_{r}}\left(\sum_{\substack{\tilde{k} \in \mathbb{Z}_{0} \\
0 \leq \tilde{k} \leq m}} f_{1, k-\tilde{k}}\right)\left(f_{2, k}-f_{2, k+L}\right)\left(f_{3, k}-f_{3, k+L}\right), \\
& I_{2}=\sum_{k \in \mathbb{Z}_{r}}\left(\sum_{\substack{\tilde{k} \in \mathbb{Z}_{0} \\
0 \leq \tilde{k} \leq m}} f_{1, k-\tilde{k}}\right)\left(f_{2, k+L}-f_{2, k+2 L}\right)\left(f_{3, k}-f_{3, k+L}\right), \\
& I_{3}=\sum_{k \in \mathbb{Z}_{r}}\left(\sum_{\substack{\tilde{k} \in \mathbb{Z}_{0} \\
0 \leq \hat{k} \leq m}} f_{1, k-\tilde{k}}\right)\left(f_{2, k}-f_{2, k+L}\right)\left(f_{3, k+L}-f_{3, k+2 L}\right), \\
& I_{4}=\sum_{k \in \mathbb{Z}_{r}}\left(\sum_{\substack{\tilde{k} \in \mathbb{Z}_{0} \\
0 \leq \hat{k} \leq m}} f_{1, k-\tilde{k}}\right) f_{2, k+2 L}\left(f_{3, k}-f_{3, k+L}\right), \\
& I_{5}=\sum_{k \in \mathbb{Z}_{r}}\left(\sum_{\substack{\tilde{k} \in \mathbb{Z}_{0} \\
0 \leq \hat{k} \leq m}} f_{1, k-\tilde{k}}\right)\left(f_{2, k}-f_{2, k+L}\right) f_{3, k+2 L} .
\end{aligned}
$$


Therefore,

$$
I_{1} \leq \sup _{k}\left|\sum_{\substack{\tilde{k} \in \mathbb{Z}_{0} \\ 0 \leq \tilde{k} \leq m}} f_{1, k-\tilde{k}}\right|\left(\sum_{k}\left|f_{2, k}-f_{2, k+L}\right|^{2}\right)^{\frac{1}{2}}\left(\sum_{k}\left|f_{3, k}-f_{3, k+L}\right|^{2}\right)^{\frac{1}{2}}
$$

and thus for $\frac{1}{q_{1}}+\frac{1}{q_{2}}+\frac{1}{q_{3}}=1$ with $q_{1}$ very large, $q_{2}>p_{3}^{\prime}$, and $q_{3}>p_{3}$, we have

$$
\begin{aligned}
\int I_{1} d x & \leq\left\|\sup _{k}\left|\sum_{\tilde{k} \in \mathbb{Z}_{0}, 0 \leq \tilde{k} \leq m} f_{1, k-\tilde{k}}\right|\right\| \prod_{q_{1}=2}^{3}\left\|\left(\sum_{k}\left|f_{j, k}-f_{j, k+L}\right|^{2}\right)^{\frac{1}{2}}\right\|_{q_{j}} \\
& \leq C_{q_{1}}\left\|\sum_{k} f_{1, k}\right\| \prod_{q_{1}} \prod_{j=2}^{3}\left\|\left(\sum_{k}\left|f_{j, k}-f_{j, k+L}\right|^{2}\right)^{\frac{1}{2}}\right\|_{q_{j}},
\end{aligned}
$$

where the $L^{q_{1}}$ norm estimate above is a consequence of the Carleson-Hunt theorem [3], [10].

To control the product of the last three terms in (5.5) we will need the following lemma.

Lemma 15. Let $\mu \geq 0, j \in\{2,3\}, T$ be a tree of type $j$ and $T \subset S_{\mu}$, then

$$
\begin{aligned}
& \left\|\left(\sum_{(k, n) \in T}\left|\phi_{1, k, n}^{*} \psi_{1, k}^{*}\left(f_{1} * \Phi_{1, k, T}\right)\right|^{2}\right)^{\frac{1}{2}}\right\|_{B M O} \leq C 2^{-\frac{\mu}{p_{1}^{\prime}}}, \\
& \left\|\left(\sum_{(k, n) \in T}\left|\phi_{1, k, n}^{*} \psi_{1, k}^{*}\left(f_{1} * \Phi_{1, k, T}\right)\right|^{2}\right)^{\frac{1}{2}}\right\|_{2} \leq C 2^{-\frac{\mu}{p_{1}^{\prime}}}\left|I_{T}\right|^{\frac{1}{2}},
\end{aligned}
$$

where $C$ is a constant independent of $m, \mu, T$ and $f_{1}$

Proof. We prove (5.6) first. Let $J=\left[2^{k_{J}} n_{J}, 2^{k_{J}}\left(n_{J}+1\right)\right]$ and $T_{J}=$ $\left\{(k, n) \in T: I_{k, n} \subset J\right\}$. Then we have

$$
\begin{aligned}
& \inf _{c} \int_{J} \mid\left(\sum_{(k, n) \in T}\left|\phi_{1, k, n}^{*}(x) \psi_{1, k}^{*}(x)\left(f_{1} * \Phi_{1, k, T}\right)(x)\right|^{2}\right)^{\frac{1}{2}}-c \mid d x \\
& \leq \int_{J}\left(\sum_{(k, n) \in T_{J}}\left|\phi_{1, k, n}^{*}(x) \psi_{1, k}^{*}(x)\left(f_{1} * \Phi_{1, k, T}\right)(x)\right|^{2}\right)^{\frac{1}{2}} d x \\
&+\int_{J}\left(\sum_{\substack{k, n) \in T \backslash T_{J} \\
k \leq k_{J}}}\left|\phi_{1, k, n}^{*}(x) \psi_{1, k}^{*}(x)\left(f_{1} * \Phi_{1, k, T}\right)(x)\right|^{2}\right)^{\frac{1}{2}} d x \\
&\left.+\left.\inf _{c} \int_{J}\left|\sum_{\substack{(k, n) \in T \backslash T_{J} \\
k>k_{J}}}\right| \phi_{1, k, n}^{*}(x) \psi_{1, k}^{*}(x)\left(f_{1} * \Phi_{1, k, T}\right)(x)\right|^{2}\right)^{\frac{1}{2}}-c \mid d x \\
&:=R_{1}+R_{2}+R_{3} .
\end{aligned}
$$


For $R_{1}$, it is easy to see that

$$
R_{1} \leq\left\|\left(\sum_{(k, n) \in T_{J}}\left|\phi_{1, k, n}^{*} \psi_{1, k}^{*}\left(f_{1} * \Phi_{1, k, T}\right)\right|^{2}\right)^{\frac{1}{2}}\right\|_{p_{1}}|J|^{1-\frac{1}{p_{1}}} \leq C 2^{-\frac{\mu}{p_{1}^{*}}}|J| .
$$

For $R_{2}$, since $p_{1}<2$ we have

$$
\begin{aligned}
R_{2} & \leq\left(\sum_{\substack{k, n) \in T \backslash T_{J} \\
k \leq k_{J}}}\left\|\phi_{1, k, n}^{*} \psi_{1, k}^{*}\left(f_{1} * \Phi_{1, k, T}\right)\right\|_{L^{p_{1}(J)}}^{p_{1}}\right) \\
& \leq\left(\sum_{\substack{k, n) \in T \backslash T_{J} \\
k \leq k_{J}}} \frac{C\left\|\phi_{1, k, n}^{*} \psi_{1, k}^{*}\left(f_{1} * \Phi_{1, k, T}\right)\right\|_{L^{p_{1}}(J)}^{p_{1}}}{\left(1+2^{-k} \operatorname{dist}\left(J, I_{k, n}\right)\right)^{N}}\right)^{\frac{1}{p_{1}}}|J|^{1-\frac{1}{p_{1}}} \\
& \leq 2^{-\frac{\mu}{p_{1}^{\prime}}}\left(\sum_{\substack{(k, n) \in T \backslash T_{J} \\
k \leq k_{J}}} \frac{C\left|I_{k, n}\right|}{\left(1+2^{-k} \operatorname{dist}\left(J, I_{k, n}\right)\right)^{N}}\right)^{\frac{1}{p_{1}}}|J|^{1-\frac{1}{p_{1}}} \leq C 2^{-\frac{\mu}{p_{1}^{\prime}}}|J| .
\end{aligned}
$$

For $R_{3}$, we dominate it by

$$
\begin{aligned}
& \left(\inf _{c} \int_{J}\left|\left(\sum_{\substack{(k, n) \in T \backslash T_{J} \\
k>k_{J}}}\left|\phi_{1, k, n}^{*}(x) \psi_{1, k}^{*}(x)\left(f_{1} * \Phi_{1, k, T}\right)(x)\right|^{2}\right)^{\frac{1}{2}}-c\right|^{2} d x\right)^{\frac{1}{2}}|J|^{\frac{1}{2}} \\
& \leq\left(\left.\inf _{c} \int_{J}\left|\sum_{\substack{(k, n) \in T \backslash T_{J} \\
k>k_{J}}}\right| \phi_{1, k, n}^{*}(x) \psi_{1, k}^{*}(x)\left(f_{1} * \Phi_{1, k, T}\right)(x)\right|^{2}-\left.c\right|^{2} d x\right)^{\frac{1}{2}}|J|^{\frac{1}{2}} \\
& \leq C\left(\int_{J_{(k, n) \in T \backslash T_{J}}} \sum_{\substack{k>k_{J}\\
}}\left|\left(\left(\phi_{1, k, n}^{*}(x) \psi_{1, k}^{*}(x) e^{-2 \pi i c\left(\omega_{1, k, T}\right) x}\left(f_{1} * \Phi_{1, k, T}\right)(x)\right)^{2}\right)\right| d x\right)^{\frac{1}{2}}|J| \\
& \leq C\left(\int_{J} \sum_{\substack{(k, n) \in T \backslash T_{J} \\
k>k_{J}}} 2^{-k}\left|\phi_{1, k, n}^{*}(x) \psi_{1, k}^{*}(x)\left(f_{1} * \Phi_{1, k, T}\right)(x)\right|^{2} d x\right)^{\frac{1}{2}}|J| \\
& +C\left(\int_{J} \sum_{\substack{k, n) \in T \backslash T_{J} \\
k>k_{J}}}\left|\phi_{1, k, n}^{*}(x) \psi_{1, k}^{*}(x)\left(f_{1} * \Phi_{1, k, T}\right)(x)\right|\right. \\
& \left.\cdot\left|\phi_{1, k, n}^{*}(x) \psi_{1, k}^{*}(x)\left(e^{-2 \pi i c\left(\omega_{1, k, T}\right)(\cdot)}\left(f_{1} * \Phi_{1, k, T}\right)(\cdot)\right)^{\prime}(x)\right| d x\right)^{\frac{1}{2}}|J| \\
& :=R_{31}+R_{32} \text {. }
\end{aligned}
$$


Lemma 14 and interpolation give that for $q \geq p_{1}$ we have

$$
\left\|\phi_{1, k, n}^{*} \psi_{1, k}^{*}\left(f_{1} * \Phi_{1, k, T}\right)\right\|_{q} \leq C 2^{-\eta \mu} 2^{-\frac{\mu}{p_{1}^{\prime}}}\left|I_{k, n}\right|^{\frac{1}{q}}
$$

Thus, using Hölder's inequality, $R_{31}$ is estimated by

$$
\begin{gathered}
\left(\sum_{\substack{(k, n) \in T \backslash T_{J} \\
k>k_{J}}} \frac{C 2^{-k}}{\left(1+2^{-k} \operatorname{dist}\left(J, I_{k, n}\right)\right)^{N}}\left\|\phi_{1, k, n}^{*} \psi_{1, k}^{*}\left(f_{1} * \Phi_{1, k, T}\right)\right\|_{4}^{2}|J|^{\frac{1}{2}}\right)^{\frac{1}{2}}|J| \\
\quad \leq C 2^{-\eta \mu_{2}} 2^{-\frac{\mu}{p_{1}^{\prime}}}\left(\sum_{\substack{(k, n) \in T \backslash T_{J} \\
k>k_{J}}} \frac{2^{-k}\left|I_{k, n}\right|^{\frac{1}{2}}}{\left(1+2^{-k} \operatorname{dist}\left(J, I_{k, n}\right)\right)^{N}}|J|^{\frac{1}{2}}\right)^{\frac{1}{2}}|J| \\
\leq C 2^{-\eta \mu_{2}} 2^{-\frac{\mu}{p_{1}^{\prime}}}|J|
\end{gathered}
$$

and $R_{32}$ is estimated by

$$
\begin{gathered}
C \sum_{\substack{(k, n) \in T \backslash T_{J} \\
k>k_{J}}}\left\|\phi_{1, k, n}^{*} \psi_{1, k}^{*}\left(f_{1} * \Phi_{1, k, T}\right)\right\|_{L^{p_{1}^{\prime}(J)}} \\
\left.\left.\cdot\left\|\phi_{1, k, n}^{*} \psi_{1, k}^{*}\left(e^{-2 \pi i c\left(\omega_{1, k, T}\right)(\cdot)}\left(f_{1} * \Phi_{1, k, T}\right)(\cdot)\right)^{\prime}\right\|\right|_{p_{1}}\right)^{\frac{1}{2}}|J| \\
\leq C 2^{-\eta \mu_{2}} 2^{-\frac{\mu}{p_{1}^{\prime}}}\left(\sum_{\substack{k, n) \in T \backslash T_{J} \\
k>k_{J}}} \frac{2^{-\frac{k}{p_{1}^{\prime}\left(p_{1}^{\prime}+1\right)}}}{\left(1+2^{-k} \operatorname{dist}\left(J, I_{k, n}\right)\right)^{N}}|J|^{\frac{1}{p_{1}^{\prime}\left(p_{1}^{\prime}+1\right)}}\right)^{\frac{1}{2}}|J| \\
\leq C 2^{-\eta \mu_{2}} 2^{-\frac{\mu}{p_{1}^{\prime}}}|J| .
\end{gathered}
$$

This completes the proof of (5.6). Now (5.7) follows from (5.6) and interpolation.

Using this result, we obtain the following lemma.

Lemma 16. Let $\mu \geq 0, j \in\{2,3\}, T$ be a tree of type $j$ and $T \subset S_{\mu}$, then

$$
\begin{aligned}
& \left\|\sum_{k} f_{1, k}\right\|_{2} \leq C 2^{-\frac{\mu}{p_{1}^{\prime}}}\left|I_{T}\right|^{\frac{1}{2}}, \\
& \left\|e^{-2 \pi i c\left(\omega_{1, T}\right)(\cdot)} \sum_{k} f_{1, k}\right\|_{B M O} \leq C 2^{-\frac{\mu}{p_{1}^{\prime}}},
\end{aligned}
$$

where $C$ is a constant independent of $m, \mu, T$ and $f_{1}$. 
Proof. The proof of (5.9) follows from (5.7), since

$$
\begin{aligned}
\left\|\sum_{k} f_{1, k}\right\|_{2}^{2} & =\left\|\sum_{k} \sum_{n \in T_{k}} \phi_{1, k, n} \psi_{1, k}\left(f_{1} * \Phi_{1, k, T}\right)\right\|_{2}^{2} \\
& \leq \sum_{k} \sum_{n \in T_{k}}\left\|\phi_{1, k, n} \psi_{1, k}\left(f_{1} * \Phi_{1, k, T}\right)\right\|_{2}^{2} \leq C 2^{-\frac{2 \mu}{p_{1}^{\prime}}}\left|I_{T}\right| .
\end{aligned}
$$

Now we prove (5.10). Let $J=\left[2^{k_{J}} n_{J}, 2^{k_{J}}\left(n_{J}+1\right)\right]$ for some $k_{J} \in \mathbb{Z}$. Define $T_{J}:=\left\{(k, n) \in T: I_{k, n} \subset J\right\}$. Then

$$
\begin{aligned}
& |J|^{-1} \inf _{c} \int_{J}\left|\sum_{(k, n) \in T} \phi_{1, k, n}(x) \psi_{1, k}(x)\left(f_{1} * \Phi_{1, k, T}\right)(x) e^{-2 \pi i c\left(\omega_{1, T}\right) x}-c\right| d x \\
& \leq J_{1}+J_{2}+J_{3},
\end{aligned}
$$

where

$$
\begin{aligned}
& J_{1}=|J|^{-1} \int_{J}\left|\sum_{(k, n) \in T_{J}} \phi_{1, k, n}(x) \psi_{1, k}(x)\left(f_{1} * \Phi_{1, k, T}\right)(x)\right| d x \\
& J_{2}=|J|^{-1} \int_{J}\left|\sum_{(k, n) \in T \backslash T_{J}, k \leq k_{J}} \phi_{1, k, n}(x) \psi_{1, k}(x)\left(f_{1} * \Phi_{1, k, T}\right)(x)\right| d x \\
& J_{3}=|J|^{-1} \inf _{c} \int_{J}\left|\sum_{(k, n) \in T, k>k_{J}} \phi_{1, k, n}(x) \psi_{1, k}(x)\left(f_{1} * \Phi_{1, k, T}\right)(x) e^{-2 \pi i c\left(\omega_{1, T}\right) x}-c\right| d x .
\end{aligned}
$$

Since $T_{J}$ is a union of trees of type 2 or 3 , we have

$$
\begin{aligned}
J_{1} & \leq|J|^{-\frac{1}{2}}\left\|\sum_{(k, n) \in T_{J}} \phi_{1, k, n} \psi_{1, k}\left(f_{1} * \Phi_{1, k, T}\right)\right\|_{2} \\
& \leq|J|^{-\frac{1}{2}}\left(\sum_{(k, n) \in T_{J}}\left\|\phi_{1, k, n}^{*} \psi_{1, k}^{*}\left(f_{1} * \Phi_{1, k, T}\right)\right\|_{2}^{2}\right)^{\frac{1}{2}} \leq C 2^{-\frac{\mu}{p_{1}^{\prime}}}
\end{aligned}
$$

which proves the required estimate for $J_{1}$.

For $J_{2}$, we use (5.8) to obtain

$$
\begin{aligned}
J_{2} & \leq|J|^{-\frac{1}{2}} \sum_{\substack{(k, n) \in T \backslash T_{J} \\
k \leq k_{J}}}\left\|\phi_{1, k, n} \psi_{1, k}\left(f_{1} * \Phi_{1, k, T}\right)\right\|_{L^{2}(J)} \\
& \leq C|J|^{-\frac{1}{2}} \sum_{\substack{(k, n) \in T \backslash T_{J} \\
k \leq k_{J}}}\left(\int_{J} \frac{\left|\phi_{1, k, n}^{*}(x) \psi_{1, k}^{*}(x)\left(f_{1} * \Phi_{1, k, T}\right)(x)\right|^{2}}{\left(1+2^{-k} \operatorname{dist}\left(x, I_{k, n}\right)\right)^{N}} d x\right)^{\frac{1}{2}}
\end{aligned}
$$




$$
\begin{aligned}
& \leq C|J|^{-\frac{1}{2}} \sum_{\substack{(k, n) \in T \backslash T_{J} \\
k \leq k_{J}}} \frac{1}{\left(1+2^{-k} \operatorname{dist}\left(J, I_{k, n}\right)\right)^{N}}\left\|\phi_{1, k, n}^{*} \psi_{1, k}^{*}\left(f_{1} * \Phi_{1, k, T}\right)\right\|_{2} \\
& \leq C 2^{-\frac{\mu}{p_{1}^{\prime}}}|J|^{-\frac{1}{2}} \sum_{\substack{(k, n) \in T \backslash T_{J} \\
k \leq k_{J}}} \frac{\left|I_{k, n}\right|^{\frac{1}{2}}}{\left(1+2^{-k} \operatorname{dist}\left(J, I_{k, n}\right)\right)^{N}} \leq C 2^{-\frac{\mu}{p_{1}^{\prime}}} .
\end{aligned}
$$

Finally we can control $J_{3}$ by

$$
\int_{J}\left|\sum_{\substack{k, n) \in T \\ k>k_{J}}}\left(\phi_{1, k, n}(x) \psi_{1, k}(x)\left(f_{1} * \Phi_{1, k, T}\right)(x) e^{-2 \pi i c\left(\omega_{1, T}\right) x}\right)^{\prime}\right| d x
$$

which is equal to

$$
\int_{J}\left|\sum_{\substack{(k, n) \in T \\ k>k_{J}}}\left(\phi_{1, k, n}(x) \psi_{1, k}(x)\left(f_{1} * \Phi_{1, k, T}\right)(x) e^{-2 \pi i c\left(\omega_{1, k, T}\right) x} e^{-2 \pi i\left(c\left(\omega_{1, T}\right)-c\left(\omega_{1, k, T}\right)\right) x}\right)^{\prime}\right| d x .
$$

Thus we obtain the estimate $J_{3} \leq J_{31}+J_{32}$, where $J_{31}$ is

$$
\int_{J} \sum_{(k, n) \in T, k>k_{J}}\left|\left(\phi_{1, k, n}(x) \psi_{1, k}(x)\left(f_{1} * \Phi_{1, k, T}\right)(x) e^{-2 \pi i c\left(\omega_{1, k, T}\right) x}\right)^{\prime}\right| d x,
$$

and $J_{32}$ is

$C \int_{J} \sum_{\substack{k, n) \in T \\ k>k_{J}}}\left|\phi_{1, k, n}(x) \psi_{1, k}(x)\left(f_{1} * \Phi_{1, k, T}\right)(x) e^{-2 \pi i c\left(\omega_{1, k, T}\right) x}\left(c\left(\omega_{1, T}\right)-c\left(\omega_{1, k, T}\right)\right)\right| d x$.

Since $T$ is a tree of type 2 or 3 it follows from Lemma 3 that $\mid c\left(\omega_{1, T}\right)-$ $c\left(\omega_{1, k, T}\right)|\leq 3| \omega_{1, k, T} \mid$. Thus by (5.8) we have

$$
\begin{aligned}
J_{32} & \leq C|J|^{\frac{1}{2}} \sum_{\substack{k, n) \in T \\
k>k_{J}}} 2^{-k}\left\|\phi_{1, k, n} \psi_{1, k}\left(f_{1} * \Phi_{1, k, T}\right)\right\|_{L^{2}(J)} \\
& \leq C|J|^{\frac{1}{2}} \sum_{\substack{k, n) \in T \\
k>k_{J}}} \frac{2^{-k}}{\left(1+2^{-k} \operatorname{dist}\left(J, I_{k, n}\right)\right)^{N}}\left\|\phi_{1, k, n}^{*} \psi_{1, k}^{*}\left(f_{1} * \Phi_{1, k, T}\right)\right\|_{2} \\
& \leq C 2^{-\frac{\mu}{p_{1}^{\prime}}}|J|^{\frac{1}{2}} \sum_{k>k_{J}} \sum_{n \in T_{k}} \frac{2^{-k}\left|I_{k, n}\right|^{\frac{1}{2}}}{\left(1+2^{-k} \operatorname{dist}\left(J, I_{k, n}\right)\right)^{N}} \leq C 2^{-\frac{\mu}{p_{1}^{\prime}}}
\end{aligned}
$$


For $J_{31}$, we have

$$
\begin{aligned}
& J_{31} \leq C \sum_{\substack{(k, n) \in T \\
k>k_{J}}}\left\|\left(\phi_{1, k, n} \psi_{1, k}\left(f_{1} * \Phi_{1, k, T}\right) e^{-2 \pi i c\left(\omega_{1, k, T}\right)(\cdot)}\right)^{\prime}\right\|_{L^{1}(J)} \\
& \leq C \sum_{\substack{(k, n) \in T \\
k>k_{J}}} 2^{-k}\left\|\phi_{1, k, n}^{*} \psi_{1, k}^{*}\left(f_{1} * \Phi_{1, k, T}\right)\right\|_{L^{1}(J)} \\
& +C \sum_{\substack{(k, n) \in T \\
k>k_{J}}}\left\|\phi_{1, k, n}^{*} \psi_{1, k}^{*}\left(e^{-2 \pi i c\left(\omega_{1, k, T}\right)(\cdot)}\left(f_{1} * \Phi_{1, k, T}\right)(\cdot)\right)^{\prime}\right\|_{L^{1}(J)} \\
& \leq C \sum_{\substack{(k, n) \in T \\
k>k_{J}}} \frac{2^{-k}}{\left(1+2^{-k} \operatorname{dist}\left(J, I_{k, n}\right)\right)^{N}}\left\|\phi_{1, k, n}^{*} \psi_{1, k}^{*}\left(f_{1} * \Phi_{1, k, T}\right)\right\|_{p_{1}}|J|^{1-\frac{1}{p_{1}}} \\
& +C \sum_{\substack{(k, n) \in T \\
k>k_{J}}} \frac{|J|^{1-\frac{1}{p_{1}}}\left\|\phi_{1, k, n}^{*} \psi_{1, k}^{*}\left(e^{-2 \pi i c\left(\omega_{1, k, T}\right)(\cdot)}\left(f_{1} * \Phi_{1, k, T}\right)(\cdot)\right)^{\prime}\right\|_{p_{1}}}{\left(1+2^{-k} \operatorname{dist}\left(J, I_{k, n}\right)\right)^{N}} \\
& \leq C 2^{-\eta \mu} 2^{-\frac{\mu}{p_{1}^{\prime}}} \sum_{\substack{(k, n) \in T \\
k>k_{J}}} \frac{\left|I_{k, n}\right|^{\frac{1}{p_{1}}-1}}{\left(1+2^{-k} \operatorname{dist}\left(J, I_{k, n}\right)\right)^{N}}|J|^{1-\frac{1}{p_{1}}} \leq C 2^{-\eta \mu} 2^{-\frac{\mu}{p_{1}^{\prime}}},
\end{aligned}
$$

where we used (3.6) and (3.7) (which failed at the step $\mu-1$ ) in the last two estimates above. This completes the proof of (5.10)

Now interpolate between (5.9) and (5.10) to obtain

$$
\left\|\sum_{k} f_{1, k}\right\|_{q_{1}} \leq C 2^{-\frac{\mu}{p_{1}^{\prime}}}\left|I_{T}\right|^{\frac{1}{q_{1}}}
$$

where $C$ is independent of $q_{1}$.

Next we write $\left(\sum_{k}\left|f_{j, k}-f_{j, k+L}\right|^{2}\right)^{\frac{1}{2}}$ as

$$
\left(\sum_{k}\left|\sum_{n \in T_{k}} \phi_{j, k, n} \psi_{j, k}\left(f_{j} * \Phi_{j, k, T}\right)-\sum_{n \in T_{k+L}} \phi_{j, k+L, n} \psi_{j, k+L}\left(f_{j} * \Phi_{j, k+L, T}\right)\right|^{2}\right)^{\frac{1}{2}}
$$

and thus we control

$$
\left(\sum_{k}\left|f_{j, k}-f_{j, k+L}\right|^{2}\right)^{\frac{1}{2}} \leq I_{11}^{(j)}+I_{12}^{(j)}+I_{13}^{(j)}
$$


where

$$
\begin{aligned}
& I_{11}^{(j)}=\left(\sum_{k}\left|\sum_{n \in T_{k+L}} \phi_{j, k+L, n} \psi_{j, k+L}\left(f_{j} *\left(\Phi_{j, k, T}-\Phi_{j, k+L, T}\right)\right)\right|^{2}\right)^{\frac{1}{2}} \\
& I_{12}^{(j)}=\left(\sum_{k}\left|\sum_{n \in T_{k+L}} \phi_{j, k+L, n}\left(\psi_{j, k}-\psi_{j, k+L}\right)\left(f_{j} * \Phi_{j, k, T}\right)\right|^{2}\right)^{\frac{1}{2}} \\
& I_{13}^{(j)}=\left(\sum_{k}\left|\left(\sum_{n \in T_{k}} \phi_{j, k, n}-\sum_{n \in T_{k+L}} \phi_{j, k+L, n}\right) \psi_{j, k}\left(f_{j} * \Phi_{j, k, T}\right)\right|^{2}\right)^{\frac{1}{2}}
\end{aligned}
$$

By (3.13) and (3.14) we obtain

$$
\begin{aligned}
\left\|I_{11}^{(j)}\right\|_{p_{j}} & \leq\left\|\left(\sum_{(k+L, n) \in T}\left|\phi_{j, k+L, n}^{*} \psi_{j, k+L}^{*}\left(f_{j} *\left(\Phi_{j, k, T}-\Phi_{j, k+L, T}\right)\right)\right|^{2}\right)^{\frac{1}{2}}\right\|_{p_{j}} \\
& \leq C 2^{-\frac{\mu}{p_{j}^{\prime}}\left|I_{T}\right|^{\frac{1}{p_{j}}}}
\end{aligned}
$$

Thus by Lemma 13 and interpolation, we have

$$
\left\|I_{11}^{(j)}\right\|_{q_{j}} \leq C 2^{-\frac{\mu}{p_{j}^{\prime}} \frac{p_{j}}{q_{j}}}\left|I_{T}\right|^{\frac{1}{q_{j}}}
$$

where $C$ is independent of $q_{j}$.

As in [8] and [14], we observe that

$$
\left|\psi_{j, k}-\psi_{j, k+L}\right| \leq C \sum_{J \in \Delta_{k-m}} \rho_{k-m, J}^{2} .
$$

Introduce sets $V_{k}^{+}=\left\{n \in T_{k}: n+1 \notin T_{k}\right\}$, and $V_{k}^{-}=\left\{n \in T_{k}: n-1 \notin T_{k}\right\}$. Then we have

$$
\begin{aligned}
& \left|\sum_{n \in T_{k+L}} \phi_{j, k+L, n}\left(\psi_{j, k}-\psi_{j, k+L}\right)\right| \\
& \leq \sum_{J \in \Delta_{k-m, T}} \rho_{k-m, J}^{2}+\sum_{n \in V_{k+L}^{+} \cup V_{k+L}^{-}} \phi_{j, k+L, n}^{*} \psi_{j, k+L}^{*} \\
& \quad+\sum_{n \in T_{k+L}} \phi_{j, k+L, n}^{*} \psi_{j, k+L}^{*} \frac{C}{\left(1+2^{-k+L} \operatorname{dist}\left(I_{k+L, n},\left(2 I_{T}\right)^{c}\right)\right)^{N}} \\
& \quad+C \sum_{\substack{n \in T_{k+L} \\
n \in\left(V_{k+L}^{+} \cup V_{k+L}^{-}\right)^{c}}} \phi_{j, k+L, n}^{*} \psi_{j, k+L}^{*} \sum_{\substack{J \in \Delta_{k-m} \backslash \Delta_{k-m, T} \\
J \in 2 I_{T}}} \rho_{k-m, J} .
\end{aligned}
$$


Using this, (3.15), and (3.10), we dominate $\left\|I_{12}^{(j)}\right\|_{p_{j}}$ by

$$
\begin{aligned}
& \left\|\left(\sum_{k}\left|\sum_{n \in T_{k+L}} \phi_{j, k+L, n}\left(\psi_{j, k}-\psi_{j, k+L}\right)\left(f_{j} * \Phi_{j, k, T}\right)\right|^{2}\right)^{\frac{1}{2}}\right\|_{p_{j}} \\
& \leq\left\|\left(\sum_{k} \sum_{J \in \Delta_{k-m, T}}\left|\rho_{k-m, J}\left(f_{j} * \Phi_{j, k, T}\right)\right|^{2}\right)^{\frac{1}{2}}\right\|_{p_{j}} \\
& +\left\|\left(\sum_{k} \sum_{n \in V_{k+L}^{+} \cup V_{k+L}^{-}}\left|\phi_{j, k+L, n}^{*} \psi_{j, k}^{*}\left(f_{j} * \Phi_{j, k+L, T}\right)\right|^{2}\right)^{\frac{1}{2}}\right\|_{p_{j}} \\
& +C\left\|\left(\sum_{(k+L, n) \in T} \frac{\left|\phi_{j, k+L, n}^{*} \psi_{j, k}^{*}\left(f_{j} * \Phi_{j, k+L, T}\right)\right|^{2}}{\left(1+2^{-k+L} \operatorname{dist}\left(I_{k+L, n},\left(2 I_{T}\right)^{c}\right)\right)^{N}}\right)^{\frac{1}{2}}\right\|_{p_{j}} \\
& +C\left\|\left(\sum_{k} \sum_{\substack{n \in T_{k+L} \\
n \in\left(V_{k+L}^{+} \bigcup V_{k+L}^{-}\right)^{c}}} \phi_{j, k+L, n}^{*} \psi_{j, k+L}^{*} \sum_{\substack{J \in \Delta_{k-m} \backslash \Delta_{k-m, T} \\
J \subset 2 I_{T}}} \rho_{k-m, J}\left|f_{j} * \Phi_{j, k, T}\right|^{2}\right)^{\frac{1}{2}}\right\|_{p_{j}} \\
& \leq C 2^{-\frac{\mu}{p_{j}^{\prime}}}\left|I_{T}\right|^{\frac{1}{p_{j}}}+\left(\sum_{k} \sum_{n \in V_{k+L}^{+} \cup V_{k+L}^{-}}\left\|\phi_{j, k+L, n}^{*} \psi_{j, k}^{*}\left(f_{j} * \Phi_{j, k+L, T}\right)\right\|_{p_{j}}^{p_{j}}\right)^{\frac{1}{p_{j}}} \\
& +C\left(\sum_{(k+L, n) \in T} \frac{\left\|\phi_{j, k+L, n}^{*} \psi_{j, k}^{*}\left(f_{j} * \Phi_{j, k+L, T}\right)\right\|_{p_{j}}^{p_{j}}}{\left(1+2^{-k+L} \operatorname{dist}\left(I_{k+L, n},\left(2 I_{T}\right)^{c}\right)\right)^{N}}\right)^{\frac{1}{p_{j}}} \\
& +C\left(\sum_{k} \sum_{n \in T_{k+L}} \sum_{J \in \Delta_{k-m} \backslash \Delta_{k-m, T}} \frac{\left\|\phi_{j, k+L, n}^{*} \psi_{j, k+L}^{*}\left(f_{j} * \Phi_{j, k, T}\right)\right\|_{p_{j}}^{p_{j}}}{\left(1+2^{-k+m} \operatorname{dist}\left(I_{k, n}, J\right)\right)^{N}}\right)^{\frac{1}{p_{j}}} \\
& n \in\left(V_{k+L}^{+} \cup V_{k+L}^{-}\right)^{c} \quad J \subset 2 I_{T} \\
& \leq C 2^{-\frac{\mu}{p_{j}^{\prime}}}\left|I_{T}\right|^{\frac{1}{p_{j}}}+C 2^{-\frac{\mu}{p_{j}^{\prime}}}\left(\sum_{k} \sum_{n \in V_{k+L}^{+} \cup V_{k+L}^{-}}\left|I_{k, n}\right|\right)^{\frac{1}{p_{j}}} \\
& +C 2^{-\eta \mu} 2^{-\frac{\mu}{p_{j}^{\prime}}}\left(\sum_{(k+L, n) \in T} \frac{\left|I_{k+L, n}\right|}{\left(1+2^{-k+L} \operatorname{dist}\left(I_{k+L, n},\left(2 I_{T}\right)^{c}\right)\right)^{N}}\right)^{\frac{1}{p_{j}}} \\
& +C 2^{-\eta \mu} 2^{-\frac{\mu}{p_{j}^{\prime}}}\left(\sum_{k} \sum_{n \in T_{k+L}} \sum_{J \in \Delta_{k-m} \backslash \Delta_{k-m, T}} \frac{2^{k}}{\left(1+2^{-k+m} \operatorname{dist}\left(I_{k, n}, J\right)\right)^{N}}\right)^{\frac{1}{p_{j}}} \\
& n \in\left(V_{k+L}^{+} \cup V_{k+L}^{-}\right)^{c} \quad J \subset 2 I_{T} \\
& \leq C 2^{-\frac{\mu}{p_{j}^{\prime}}}\left|I_{T}\right|^{\frac{1}{p_{j}}}+C 2^{-\frac{\mu}{p_{j}^{\prime}}}\left(\sum_{k} \sum_{\substack{J \in \Delta_{k}-m \backslash \Delta_{k-m, T} \\
J \subset 2 I_{T}}} 2^{k-m}\right)^{\frac{1}{p_{j}}} \leq C 2^{-\frac{\mu}{p_{j}^{\prime}}}\left|I_{T}\right|^{\frac{1}{p_{j}}}
\end{aligned}
$$


This gives us an $L^{p_{j}}$ estimate for $I_{12}^{(j)}$. As in [8], it is easy to prove that $\left\|I_{12}^{(j)}\right\|_{B M O} \leq C$. Hence by interpolation, we obtain

$$
\left\|I_{12}^{(j)}\right\|_{q_{j}} \leq C 2^{-\frac{\mu}{p_{j}^{\prime}} \frac{p_{j}}{q_{j}}}\left|I_{T}\right|^{\frac{1}{q_{j}}}
$$

where $C$ is independent of $q_{j}$.

We now control $\left\|I_{13}^{(j)}\right\|_{p_{j}}$ by

$$
\begin{aligned}
& \left(\sum_{k}\left\|\left(\sum_{n \in T_{k}} \phi_{j, k, n}-\sum_{n \in T_{k+L}} \phi_{j, k+L, n}\right) \psi_{j, k}\left(f_{j} * \Phi_{j, k, T}\right)\right\|_{p_{j}}^{p_{j}}\right)^{\frac{1}{p_{j}}} \\
& \leq\left(\sum_{k} \sum_{n^{\prime}}\left\|\left(\sum_{n \in T_{k}} \phi_{j, k, n}-\sum_{n \in T_{k+L}} \phi_{j, k+L, n}\right) \psi_{j, k}\left(f_{j} * \Phi_{j, k, T}\right)\right\|_{L^{p_{j}\left(I_{k, n^{\prime}}\right)}}^{p_{j}}\right)^{\frac{1}{p_{j}}} \\
& \leq I_{13}^{(j 1)}+\left\|\phi_{j, k_{T}, n_{T}}^{*} \psi_{j, k_{T}}^{*}\left(f_{j} * \Phi_{j, k_{T}, T}\right)\right\|_{p_{j}} \\
& \leq I_{13}^{(j 1)}+C 2^{-\eta \mu_{2}} 2^{-\frac{\mu}{p_{j}^{\prime}}}\left|I_{T}\right|^{\frac{1}{p_{j}}}
\end{aligned}
$$

in view of (3.10), where we set $I_{13}^{(j 1)}$ to be the expression

$$
\left(\sum_{\substack{k \neq k_{T} \\ n^{\prime}}}\left\|\left|\sum_{n \in T_{k}} \phi_{j, k, n}-\sum_{n \in T_{k+L}} \phi_{j, k+L, n}\right|^{\frac{1}{2}}\right\|_{L^{\infty}\left(I_{k, n^{\prime}}\right)}\left\|\sum_{n \in T_{k}} \phi_{j, k, n}^{*} \psi_{j, k}^{*}\left(f_{j} * \Phi_{j, k, T}\right)\right\|_{L^{p_{j}\left(I_{k, n^{\prime}}\right)}}^{p_{j}}\right)^{\frac{1}{p_{j}}} .
$$

Note that

$$
\begin{aligned}
\| \sum_{n \in T_{k}} \phi_{j, k, n}^{*} & \psi_{j, k}^{*}\left(f_{j} * \Phi_{j, k, T}\right) \|_{L^{p_{j}\left(I_{k, n^{\prime}}\right)}}^{p_{j}} \\
& \leq \int_{I_{k, n^{\prime}}}\left(\sum_{n \in T_{k}} \phi_{j, k, n}^{*}(x)\right)^{p_{j}}\left|\psi_{j, k}^{*}(x)\left(f_{j} * \Phi_{j, k, T}\right)(x)\right|^{p_{j}} d x \\
& \leq \sum_{n \in T_{k}}\left\|\phi_{j, k, n}^{*}\right\|\left\|_{L^{\infty}\left(I_{k, n^{\prime}}\right)}\right\| \phi_{j, k, n}^{*} \psi_{j, k}^{*}\left(f_{j} * \Phi_{j, k, T}\right) \|_{p_{j}}^{p_{j}} \\
& \leq C 2^{-p_{j} \eta \mu_{2}} 2^{-\frac{p_{j} \mu}{p_{j}^{\prime}}}\left|I_{k, n}\right| \sum_{n \in T_{k}}\left\|\phi_{j, k, n}^{*}\right\|_{L^{\infty}\left(I_{k, n^{\prime}}\right)} \\
& \leq C 2^{-p_{j} \eta \mu_{2}} 2^{-\frac{p_{j} \mu}{p_{j}^{\prime}}}\left|I_{k, n}\right| .
\end{aligned}
$$

Thus, we have

$$
I_{13}^{(j 1)} \leq C 2^{-\eta \mu} 2^{-\frac{\mu}{p_{j}^{\prime}}}\left(\sum_{k<k_{T}} \sum_{n^{\prime}}\left\|\left|\sum_{n \in T_{k}} \phi_{j, k, n}-\sum_{n \in T_{k+L}} \phi_{j, k+L, n}\right|^{\frac{1}{2}}\right\|_{L^{\infty}\left(I_{k, n^{\prime}}\right)} 2^{k}\right)^{\frac{1}{p_{j}}} .
$$


We next observe that

$$
\left|\sum_{n \in T_{k}} \phi_{j, k, n}-\sum_{n \in T_{k+L}} \phi_{j, k+L, n}\right| \leq \sum_{n \in W_{k+L}} \phi_{j, k+L, n}^{*}+\sum_{n \in V_{k+L}^{+} \cup V_{k+L}^{-}} \phi_{j, k+L, n}^{*},
$$

where $W_{k+L}$ is the set

$\left\{n \in \mathbb{Z}:(k, n) \notin T\right.$ but there exists $\left(k+L, n^{\prime}\right) \in T$ such that $\left.I_{k, n} \subset I_{k+L, n^{\prime}}\right\}$.

Note that by the convexity of $T$, the set $\bigcup_{k} \bigcup_{n \in W_{k+L}}\left\{I_{k, n}\right\}$ is a set of pairwise disjoint intervals. Hence, we have

$$
\begin{aligned}
I_{13}^{(j 1)} & \leq C 2^{-\eta \mu_{2}} 2^{-\frac{\mu}{p_{j}^{\prime}}}\left(\sum_{k<k_{T}} \sum_{n^{\prime}} \sum_{n \in W_{k+L} \cup V_{k+L}^{+} \cup V_{k+L}^{-}} 2^{k}\left\|\phi_{j, k+L, n}^{*}\right\|_{L^{\infty}\left(I_{k, n^{\prime}}\right)}\right)^{\frac{1}{p_{j}}} \\
& \leq C 2^{-\eta \mu_{2}} 2^{-\frac{\mu}{p_{j}^{\prime}}}\left(\sum_{k<k_{T}} \sum_{n \in W_{k+L} \cup V_{k+L}^{+} \cup V_{k+L}^{-}}\left|I_{k, n}\right|\right)^{\frac{1}{2}} \leq C 2^{-\eta \mu_{2}} 2^{-\frac{\mu}{p_{j}^{\prime}}}\left|I_{T}\right|^{\frac{1}{p_{j}}}
\end{aligned}
$$

Therefore, we obtain

$$
\left\|I_{13}^{(j)}\right\|_{p_{j}} \leq C 2^{-\eta \mu_{2}} 2^{-\frac{\mu}{p_{j}^{\prime}}}\left|I_{T}\right|^{\frac{1}{p_{j}}}
$$

As in [8], we have that $\left\|I_{13}^{(j)}\right\|_{B M O} \leq C$. Thus, by interpolation, it follows that

$$
\left\|I_{13}^{(j)}\right\|_{q_{j}} \leq C 2^{-\frac{\mu}{p_{j}^{\prime}} \frac{p_{j}}{q_{j}}}\left|I_{T}\right|^{\frac{1}{q_{j}}}
$$

where $C$ is independent of $q_{j}$. Therefore, by (5.11)-(5.14), we obtain

$$
\left\|I_{1}\right\|_{1} \leq C_{q_{1}} 2^{-\left(\frac{1}{p_{1}^{\prime}}+\frac{1}{p_{2}^{\prime}} \frac{p_{2}}{q_{2}}+\frac{1}{p_{3}^{\prime}} \frac{p_{3}}{q_{3}}\right)}\left|I_{T}\right| .
$$

Similarly for $j=2$ and $j=3$ we get

$$
\left\|I_{j}\right\|_{1} \leq C_{q_{1}} 2^{-\left(\frac{1}{p_{1}^{\prime}}+\frac{1}{p_{2}^{\prime}} \frac{p_{2}}{q_{2}}+\frac{1}{p_{3}^{\prime}} \frac{p_{3}}{q_{3}}\right)}\left|I_{T}\right|
$$

Now we write

$$
I_{4}=I_{41}+I_{42}+I_{43}+I_{44},
$$

where $I_{41}$ is

$$
\sum_{k \in \mathbb{Z}_{r}}\left(\sum_{\substack{\tilde{\tilde{k}} \in \mathbb{Z}_{0} \\ 0 \leq \hat{k} \leq m-3 L}} f_{1, k-\tilde{k}}\right) f_{2, k+2 L}\left(\sum_{n \in T_{k+L}} \phi_{3, k+L, n} \psi_{3, k+L}\left(f_{3} *\left(\Phi_{3, k, T}-\Phi_{3, k+L, T}\right)\right)\right),
$$

$I_{42}$ is

$$
\sum_{k \in \mathbb{Z}_{r}}\left(\sum_{\substack{\tilde{k} \in \mathbb{Z}_{0} \\ 0 \leq \tilde{k} \leq m-3 L}} f_{1, k-\tilde{k}}\right) f_{2, k+2 L}\left(\sum_{n \in T_{k+L}} \phi_{3, k+L, n}\left(\psi_{3, k}-\psi_{3, k+L}\right)\left(f_{3} * \Phi_{3, k, T}\right)\right),
$$


$I_{43}$ is

$$
\sum_{k \in \mathbb{Z}_{r}}\left(\sum_{\substack{\tilde{\tilde{\kappa}} \in \mathbb{Z}_{0} \\ 0 \leq \hat{k} \leq m-3 L}} f_{1, k-\tilde{k}}\right) f_{2, k+2 L}\left(\sum_{n \in T_{k}} \phi_{3, k, n}-\sum_{n \in T_{k+L}} \phi_{3, k+L, n}\right) \psi_{3, k}\left(f_{3} * \Phi_{3, k, T}\right),
$$

and $I_{44}$ is

$$
\sum_{k \in \mathbb{Z}_{r}}\left(\sum_{\substack{\tilde{k} \in \mathbb{Z}_{0} \\ m-3 L \leq \tilde{k} \leq m}} f_{1, k-\tilde{k}}\right) f_{2, k+2 L}\left(f_{3, k}-f_{3, k+L}\right) .
$$

We now observe the fact (see [8]) that the integral of $I_{41}$ is zero. For $I_{42}$, we control $\left|I_{42}\right|$ by

$$
\begin{aligned}
& \sum_{k \in \mathbb{Z}_{r}}\left|\sum_{\substack{\tilde{k} \in \mathbb{Z}_{0} \\
0 \leq \tilde{k} \leq m-3 L}} f_{1, k-\tilde{k}} \| \sum_{n \in T_{k+L}} \phi_{2, k+L, n}\left(\psi_{2, k}-\psi_{2, k+L}\right)\right|^{\frac{1}{2}}\left|f_{2} * \Phi_{2, k+2 L, T}\right| \\
& \cdot\left|\sum_{n \in T_{k+L}} \phi_{3, k+L, n}\left(\psi_{3, k}-\psi_{3, k+L}\right)\right|^{\frac{1}{2}}\left|f_{3} * \Phi_{3, k+2 L, T}\right| \\
& \leq \sup _{k \in \mathbb{Z}_{r}}\left|\sum_{\substack{\tilde{k} \in \mathbb{Z}_{0} \\
0 \leq \tilde{k} \leq m-3 L}} f_{1, k-\tilde{k}}\right|\left(\sum_{k \in \mathbb{Z}_{r}}\left|\sum_{n \in T_{k+L}} \phi_{2, k+L, n}\left(\psi_{2, k}-\psi_{2, k+L}\right)\right|\left|f_{2} * \Phi_{2, k+2 L, T}\right|^{2}\right)^{\frac{1}{2}} \\
& \cdot\left(\sum_{k \in \mathbb{Z}_{r}}\left|\sum_{n \in T_{k+L}} \phi_{3, k+L, n}\left(\psi_{3, k}-\psi_{3, k+L}\right)\right|\left|f_{3} * \Phi_{3, k, T}\right|^{2}\right)^{\frac{1}{2}} \cdot
\end{aligned}
$$

As for the estimates for $I_{1}$ and $I_{12}^{(j)},\left\|I_{42}\right\|_{1}$ is dominated by

$$
\begin{gathered}
C_{q_{1}}\left\|\sum_{k \in \mathbb{Z}_{r}} f_{1, k}\right\|\left\|_{q_{1}}\right\|\left(\sum_{k \in \mathbb{Z}_{r}}\left|\sum_{n \in T_{k+L}} \phi_{2, k+L, n}\left(\psi_{2, k}-\psi_{2, k+L}\right)\right|\left|f_{2} * \Phi_{2, k+2 L, T}\right|^{2}\right)^{\frac{1}{2}} \|_{q_{2}} \\
\cdot\left\|\left(\sum_{k \in \mathbb{Z}_{r}}\left|\sum_{n \in T_{k+L}} \phi_{3, k+L, n}\left(\psi_{3, k}-\psi_{3, k+L}\right)\right|\left|f_{3} * \Phi_{3, k, T}\right|^{2}\right)^{\frac{1}{2}}\right\|_{q_{3}} \\
\leq C_{q_{1}} 2^{-\left(\frac{1}{p_{1}^{\prime}}+\frac{1}{p_{2}^{\prime}} \frac{p_{2}}{q_{2}}+\frac{1}{p_{3}^{\prime}} \frac{p_{3}}{q_{3}}\right)}\left|I_{T}\right| .
\end{gathered}
$$

For $I_{43}$, we control $\left|I_{43}\right|$ by

$$
\begin{aligned}
& \sup _{k \in \mathbb{Z}_{r}}\left|\sum_{\substack{\tilde{k} \in \mathbb{Z}_{0} \\
0 \leq \tilde{k} \leq m-3 L}} f_{1, k-\tilde{k}}\right|\left(\sum_{k}\left|\sum_{n \in T_{k}} \phi_{2, k, n}-\sum_{n \in T_{k+L}} \phi_{2, k+L, n}\right|\left|\psi_{2, k}\left(f_{2} * \Phi_{2, k+2 L, T}\right)\right|^{2}\right)^{\frac{1}{2}} \\
& \left(\sum_{k}\left|\sum_{n \in T_{k}} \phi_{3, k, n}-\sum_{n \in T_{k+L}} \phi_{3, k+L, n}\right|\left|\psi_{3, k}\left(f_{3} * \Phi_{3, k, T}\right)\right|^{2}\right)^{\frac{1}{2}} .
\end{aligned}
$$


Therefore, as in the estimates for $I_{1}$ and $I_{13}^{(j)}$, we obtain that $\left\|I_{43}\right\|_{1}$ is controlled by

$$
\begin{gathered}
C_{q_{1}}\left\|\sum_{k \in \mathbb{Z}_{r}} f_{1, k}\right\| \mid\left\|\left(\sum_{q_{1}}\left|\sum_{n \in T_{k}} \phi_{2, k, n}-\sum_{n \in T_{k+L}} \phi_{2, k+L, n}\right|\left|\psi_{2, k}\left(f_{2} * \Phi_{2, k+2 L, T}\right)\right|^{2}\right)^{\frac{1}{2}}\right\|_{q_{2}} \\
\cdot\left\|\left(\sum_{k}\left|\sum_{n \in T_{k}} \phi_{3, k, n}-\sum_{n \in T_{k+L}} \phi_{3, k+L, n}\right|\left|\psi_{3, k}\left(f_{3} * \Phi_{3, k, T}\right)\right|^{2}\right)^{\frac{1}{2}}\right\|_{q_{3}} \\
\leq C_{q_{1}} 2^{-\left(\frac{1}{p_{1}^{\prime}}+\frac{1}{p_{2}^{\prime}} \frac{p_{2}}{q_{2}}+\frac{1}{p_{3}^{\prime}} \frac{p_{3}}{q_{3}}\right)}\left|I_{T}\right| .
\end{gathered}
$$

In $I_{44}$, the index $\tilde{k}$ runs through three values. We estimate each of the three summands separately. For $\tilde{k} \in\{0, L, 2 L\}$ we have

$$
\begin{aligned}
\sum_{k \in \mathbb{Z}_{r}} \mid f_{1, k-m+\tilde{k}} f_{2, k+2 L} & \left(f_{3, k}-f_{3, k+L}\right) \mid \\
\leq & \left(\sum_{k \in \mathbb{Z}_{r}}\left|f_{1, k-m+\tilde{k}} f_{2, k+2 L}\right|^{2}\right)^{\frac{1}{2}}\left(\sum_{k \in \mathbb{Z}_{r}}\left|f_{3, k}-f_{3, k+L}\right|^{2}\right)^{\frac{1}{2}} .
\end{aligned}
$$

Therefore, we estimate $\left\|I_{44}\right\|_{1}$ by

$$
\begin{aligned}
& C\left\|\left(\sum_{k \in \mathbb{Z}_{r}}\left|f_{1, k-m+\tilde{k}} f_{2, k+2 L}\right|^{2}\right)^{\frac{1}{2}}\right\|_{p_{3}^{\prime}}\left\|\left(\sum_{k \in \mathbb{Z}_{r}}\left|f_{3, k}-f_{3, k+L}\right|^{2}\right)^{\frac{1}{2}}\right\|_{p_{3}} \\
& \leq C\left\|\left(\sum_{k \in \mathbb{Z}_{r}}\left|\sum_{n \in T_{k-m+\tilde{k}}} \phi_{1, k-m+\tilde{k}, n} \psi_{1, k-m+\tilde{k}}\left(f_{1} * \Phi_{1, k-m+\tilde{k}}\right) \psi_{2, k+2 L}\left(f_{2} * \Phi_{2, k+2 L}\right)\right|^{2}\right)^{\frac{1}{2}}\right\|_{p_{3}^{\prime}} \\
& \text {. } 2^{-\frac{\mu}{p_{3}^{\prime}}}\left|I_{T}\right|^{\frac{1}{p_{3}}} \\
& \leq C\left\|\left(\sum_{k \in \mathbb{Z}_{r}} \sum_{n \in T_{k-m+\tilde{k}}}\left|\phi_{1, k-m+\tilde{k}, n}^{*} \psi_{1, k-m+\tilde{k}}^{*}\left(f_{1} * \Phi_{1, k-m+\tilde{k}}\right)\right|^{2}\right)^{\frac{1}{2}}\right\|_{p_{3}^{\prime}} \\
& \sup _{(k-m+\tilde{k}, n) \in T}\left\|\phi_{1, k-m+\tilde{k}, n}^{*} \psi_{2, k+2 L}^{*}\left(f_{2} * \Phi_{2, k+2 L}\right)\right\|_{\infty} 2^{-\frac{\mu}{p_{3}^{\prime}}}\left|I_{T}\right|^{\frac{1}{p_{3}}} \\
& \leq C\left\|\left(\sum_{(k, n) \in T}\left|\phi_{1, k, n}^{*} \psi_{1, k}^{*}\left(f_{1} * \Phi_{1, k}\right)\right|^{2}\right)^{\frac{1}{2}}\right\|_{p_{3}^{\prime}} \\
& \text { - } \sup _{(k, n) \in T}\left\|\phi_{1, k, n}^{*} \psi_{2, k+m-\tilde{k}+2 L}^{*}\left(f_{2} * \Phi_{2, k+m-\tilde{k}+2 L}\right)\right\|_{\infty} 2^{-\frac{\mu}{p_{3}}}\left|I_{T}\right|^{\frac{1}{2}} \\
& \leq C 2^{-\left(\frac{\mu}{p_{1}^{T}}+\frac{\mu}{p_{3}^{\prime}}\right)}\left|I_{T}\right| \sup _{(k, n) \in T}\left\|\phi_{1, k, n}^{*} \psi_{2, k+m-\tilde{k}+2 L}^{*}\left(f_{2} * \Phi_{2, k+m-\tilde{k}+2 L}\right)\right\|_{\infty}
\end{aligned}
$$

where we used Lemma 15. 
Note that, by Lemma 14, we have

$$
\begin{aligned}
\sup _{(k, n) \in T} & \left\|\phi_{1, k, n}^{*} \psi_{2, k+m-\tilde{k}+2 L}^{*}\left(f_{2} * \Phi_{2, k+m-\tilde{k}+2 L}\right)\right\|_{\infty} \\
\leq & C \sup _{(k, n) \in T}\left\|\phi_{1, k, n}^{*} \psi_{2, k+m-\tilde{k}+2 L}^{*}\left(f_{2} * \Phi_{2, k+m-\tilde{k}+2 L}\right)\right\|_{p_{2}^{\prime}}^{\frac{1}{2}} \\
& \cdot\left\|\left(\phi_{1, k, n}^{*} \psi_{2, k+m-\tilde{k}+2 L}^{*}\left(e^{-2 \pi i c\left(\omega_{2, k+m-\tilde{k}+2 L, T}\right)(\cdot)}\left(f_{2} * \Phi_{2, k+m-\tilde{k}+2 L}\right)(\cdot)\right)\right)^{\prime}\right\|_{p_{2}}^{\frac{1}{2}} \\
\leq & C 2^{-\eta \mu} 2^{-\frac{\mu}{p_{2}^{\prime}}} .
\end{aligned}
$$

Thus, we obtain

$$
\left\|I_{44}\right\|_{1} \leq C 2^{-\left(\frac{1}{p_{1}^{\prime}}+\frac{1}{p_{2}^{\prime}}+\frac{1}{p_{3}^{\prime}}\right) \mu}\left|I_{T}\right|
$$

Hence, we have

$$
\left\|I_{4}\right\|_{1} \leq C_{q_{1}} 2^{-\left(\frac{1}{p_{1}^{\prime}}+\frac{1}{p_{2}^{\prime}} \frac{p_{2}}{q_{2}}+\frac{1}{p_{3}^{\prime}} \frac{p_{3}}{q_{3}}\right)}\left|I_{T}\right|
$$

Similarly, we have

$$
\left\|I_{5}\right\|_{1} \leq C_{q_{1}} 2^{-\left(\frac{1}{p_{1}^{\prime}}+\frac{1}{p_{2}^{\prime}} \frac{p_{2}}{q_{2}}+\frac{1}{p_{3}^{\prime}} \frac{p_{3}}{q_{3}}\right)}\left|I_{T}\right|
$$

This completes the proof of (3.19).

\section{Counting the trees, part I}

Having established the proof of Lemma 4, we now turn our attention to Lemma 5. The proof of this lemma will be presented in this and in the next two sections. In this section we prove (3.20) for $(i, j, \nu) \in \bigcup_{i, j \in\{2,3\}}\{(i, j, 2)\} \cup$ $\{(1,1,1)\}$. We only prove the case $(i, j, 2)$ if $i, j \in\{2,3\}$. The proof for the case $(1,1,1)$ is similar. For simplicity, we assume that (3.10) holds for $(k, n) \in T$ and $T \in \bigcup_{l} T_{\mu, i, j, l}^{2}$. Let $\mathcal{F}_{i, j, 2}=\bigcup_{l} T_{\mu, i, j, l}^{2}, \mathcal{N}_{\mathcal{F}_{i, j, 2}}(x)=$ $\sum_{T \in \mathcal{F}_{i, j, 2}} 1_{I_{T}}(x)$. It is sufficient to prove

$$
\left\|\mathcal{N}_{\mathcal{F}_{i, j, 2}}\right\|_{1} \leq C 2^{10 \eta p_{j}^{\prime} \mu} 2^{\mu} .
$$

Since $\mathcal{N}_{\mathcal{F}_{i, j, 2}}$ is integer-valued, to prove (6.1), it suffices to show that there exists $0<\varepsilon<\eta$ such that, for any $\lambda \geq 1$,

$$
\left|\left\{x \in \mathbb{R}: \mathcal{N}_{\mathcal{F}_{i, j, 2}}(x) \geq \lambda\right\}\right| \leq C_{\varepsilon} 2^{10 \eta p_{j}^{\prime} \mu} 2^{\mu} \lambda^{-1-\varepsilon} .
$$

As in [8], take $\mathcal{F}^{\prime} \subset \mathcal{F}_{i, j, 2}$ such that $\mathcal{N}_{\mathcal{F}^{\prime}}(x) \leq \lambda$ and

$$
\left|\left\{x \in \mathbb{R}: \mathcal{N}_{\mathcal{F}^{\prime}}(x) \geq \lambda\right\}\right|=\left|\left\{x \in \mathbb{R}: \mathcal{N}_{\mathcal{F}_{i, j, 2}}(x) \geq \lambda\right\}\right| .
$$


1110 X. LI

Let $A=\lambda^{\varepsilon}$. As in [8], we have

$$
\mathcal{F}^{\prime}=\left(\bigcup_{l=1}^{A^{10}} \mathcal{F}_{l}\right) \bigcup \mathcal{F}^{\prime \prime}
$$

such that

(6.3) For $T, T^{\prime} \in \mathcal{F}_{l} \quad$ and $\quad T^{\prime} \neq T, \quad\left(A I_{T} \times \omega_{i, T}\right) \cap\left(A I_{T^{\prime}} \times \omega_{i, T^{\prime}}\right)=\emptyset$,

$$
\sum_{T \in \mathcal{F}^{\prime \prime}}\left|I_{T}\right| \leq C e^{-A} \sum_{T \in \mathcal{F}_{1}}\left|I_{T}\right|
$$

For $T \in \mathcal{F}_{l}, f \in \mathcal{S}$ and $x, y \in \mathbb{R}$, define

$$
B_{T, x} f(y)=\phi_{j, k_{T}+\tilde{k}, n_{T}}^{*}(x)\left(f * \Phi_{j, k_{T}+\tilde{k}, T}\right)(x) 1_{I_{T}}(y),
$$

and

$$
\vec{B} f=\left\{B_{T, x} f\right\}_{T, x}
$$

We also define

$$
L^{q}\left(I_{T}\right)=\left\{f \in L^{q}(\mathbb{R}): \frac{1}{\left|I_{T}\right|} \int|f(x)|^{q} \varphi_{T}(x) d x<\infty\right\},
$$

where

$$
\varphi_{T}(x)=\left(1+2^{-k_{T}} \operatorname{dist}\left(x, I_{T}\right)\right)^{-N} .
$$

Let

$$
\|f\|_{L^{q}\left(I_{T}\right)}=\left(\frac{1}{\left|I_{T}\right|} \int|f(x)|^{q} \varphi_{T}(x) d x\right)^{\frac{1}{q}} .
$$

Then by the almost orthogonality lemma in [8], we have

$$
\begin{aligned}
& \|\vec{B} f\|_{L^{2}\left(\mathbb{R}, l^{2}\left(\mathcal{F}_{l}, L^{2}\left(I_{T}\right)\right)\right)} \\
& \leq\left(\int \sum_{T \in \mathcal{F}_{l}} \frac{1}{\left|I_{T}\right|} \int\left|\phi_{j, k_{T}+\tilde{k}, n_{T}}^{*}(x)\left(f * \Phi_{j, k_{T}+\tilde{k}, T}\right)(x)\right|^{2} \varphi_{T}(x) d x 1_{I_{T}}(y) d y\right)^{\frac{1}{2}} \\
& \leq\left(\sum_{T \in \mathcal{F}_{l}}\left\|\phi_{j, k_{T}+\tilde{k}, n_{T}}^{*}\left(f * \Phi_{j, k_{T}+\tilde{k}, T}\right)\right\|_{2}^{2}\right)^{\frac{1}{2}} \\
& \leq C\left(1+A^{-\frac{1}{\varepsilon}} \lambda\right)\|f\|_{2} \leq C\|f\|_{2} .
\end{aligned}
$$


On the other hand, by (4.10), we also have

$$
\begin{aligned}
\| & \vec{B} f \|_{L^{1+2 \delta}\left(\mathbb{R}, l^{\infty}\left(\mathcal{F}_{l}, L^{1+\delta}\left(I_{T}\right)\right)\right)} \\
& \leq\left(\int\left(\sup _{T \in \mathcal{F}_{l}}\left(\frac{1}{\sqrt{I_{T} \mid}} \int\left|\phi_{j, k_{T}+\tilde{k}, n_{T}}^{*}(x)\left(f * \Phi_{j, k_{T}+\tilde{k}, T}\right)(x)\right|^{1+\delta} \varphi_{T}(x) d x\right) \operatorname{1}_{I_{T}}^{\frac{1}{1+\delta}}(y)\right)^{1+2 \delta} d y\right)^{\frac{1}{1+2 \delta}} \\
& \leq\left(\int\left(\sup _{T \in \mathcal{F}_{l}} \frac{1}{\left|I_{T}\right|^{\frac{1}{1+\delta}}}\left\|\phi_{j, k_{T}+\tilde{k}, n_{T}}^{*}\left(f * \Phi_{j, k_{T}+\tilde{k}, T}\right)\right\|_{1+\delta} 1_{I_{T}}(y)\right)^{1+2 \delta} d y\right)^{\frac{1}{1+2 \delta}} \\
& \leq\left(\int\left(M_{1+\delta} f(y)\right)^{1+2 \delta} d y\right)^{\frac{1}{1+2 \delta}} \leq C\|f\|_{1+2 \delta}
\end{aligned}
$$

where $\delta>0$ is a very small number.

Therefore, by complex interpolation and the fact that $L^{q}\left(I_{T}\right) \subset L^{p}\left(I_{T}\right)$ for $q \geq p$, we obtain

$$
\|\vec{B} f\|_{L^{p_{j}}\left(\mathbb{R}, l^{p_{j}^{\prime}+\delta}\left(\mathcal{F}_{l}, L^{p_{j}-\delta}\left(I_{T}\right)\right)\right.} \leq C\|f\|_{p_{j}}
$$

Note that $\left|\phi_{j, k_{T}+\tilde{k}, n_{T}}(x)\right| \leq C \varphi_{T}(x)$, we have

$$
\int\left(\sum_{T \in \mathcal{F}_{l}}\left(\frac{1}{\left|I_{T}\right|} \int\left|\phi_{j, k_{T}+\tilde{k}, n_{T}}^{*}(x)\left(f * \Phi_{j, k_{T}+\tilde{k}, T}\right)(x)\right|^{p_{j}-\delta} d x\right)^{\frac{p_{j}^{\prime}+\delta}{p_{j}-\delta}} 1_{I_{T}}(y)\right)^{\frac{p_{j}}{p_{j}^{\prime}+\delta}} d y \leq C\|f\|_{p_{j}}^{p_{j}} .
$$

As in [8] and [12], we use a localization argument to obtain a local estimate related to $(6.6)$. In fact, let $G_{J}(f)$ be

$$
\int\left(\sum_{\substack{T \in \mathcal{F}_{l} \\ I_{T} \subset J}}\left(\frac{1}{\left|I_{T}\right|} \int\left|\phi_{j, k_{T}+\tilde{k}, n_{T}}^{*}(x)\left(f * \Phi_{j, k_{T}+\tilde{k}, T}\right)(x)\right|^{p_{j}-\delta} d x\right)^{\frac{p_{j}^{\prime}+\delta}{p_{j}-\delta}} 1_{I_{T}}(y)\right)^{\frac{p_{j}}{p_{j}^{\prime}+\delta}} d y
$$

Then we have that

$$
G_{J}(f) \leq G_{J}\left(f 1_{2 \lambda^{\varepsilon} J}\right)+G_{J}\left(f 1_{\left.\left(2 \lambda^{\varepsilon} J\right)^{c}\right)},\right.
$$

where $J \in\left\{I_{T}\right\}_{T \in \mathcal{F}^{\prime}}$. By (6.6), we have

$$
G_{J}\left(f 1_{2 \lambda^{\varepsilon} J}\right) \leq C\left\|f 1_{2 \lambda^{\varepsilon} J}\right\|_{p_{j}}^{p_{j}} \leq C \lambda^{\varepsilon}|J|\left(\inf _{x \in J} M_{p_{j}}(M f)(x)\right)^{p_{j}} .
$$


And $G_{J}\left(f 1_{\left.\left(2 \lambda^{\varepsilon} J\right)^{c}\right)}\right.$ is estimated by

$$
\begin{aligned}
\int\left(\sum _ { \substack { T \in \mathcal { F } _ { l } \\
I _ { T } \subset J } } \left(\frac{1}{\left|I_{T}\right|} \int \frac{2^{m\left(p_{j}-\delta\right)}\left(\lambda^{\varepsilon} 2^{m}\right)^{-N}}{\left(1+2^{-k_{T}} \operatorname{dist}\left(x, I_{T}\right)\right)^{N}}\right.\right. \\
\left.\left.\quad \cdot\left(\int_{\left(2 \lambda^{\varepsilon} J\right)^{c}} \frac{|f(z)| 2^{-k_{T}} d z}{\left(1+2^{-k_{T}}|x-z|\right)^{N}}\right)^{p_{j}-\delta} d x\right)^{\frac{p_{j}^{\prime}+\delta}{p_{j}-\delta}} 1_{I_{T}}(y)\right)^{\frac{p_{j}}{p_{j}^{\prime}+\delta}} d y \\
\leq \int\left(\sum_{\substack{T \in \mathcal{F}_{l} \\
I_{T} \subset J}}\left(\inf _{x \in I_{T}} M f(x)\right)^{p_{j}^{\prime}+\delta} \lambda^{-\frac{p_{j}^{\prime}+\delta}{p_{j}-\delta}} 1_{I_{T}}(y)\right)^{\frac{p_{j}}{p_{j}^{\prime}+\delta}} d y \\
\leq \frac{C}{\lambda} \int \sum_{\substack{T \in \mathcal{F}_{l} \\
I_{T} \subset J}}\left(\inf _{x \in I_{T}} M f(x)\right)^{p_{j}} 1_{I_{T}}(y) d y \leq C|J|\left(\inf _{x \in J} M_{p_{j}}(M f)(x)\right)^{p_{j}} .
\end{aligned}
$$

Hence, we obtain

$$
G_{J}(f) \leq C \lambda^{\varepsilon}|J|\left(\inf _{x \in J} M_{p_{j}}(M f)(x)\right)^{p_{j}} .
$$

Notice that

$$
\phi_{j, k_{T}+\tilde{k}, n_{T}}^{*}(x) \psi_{j, k_{T}}^{*}(x) \leq\left(1+2^{-k_{T}+m} \operatorname{dist}\left(J, E^{c}\right)\right)^{-N} .
$$

As we proved (4.6), we may sharpen the previous estimate to

$$
\begin{array}{r}
\int\left(\sum_{\substack{T \in \mathcal{F}_{l} \\
I_{T} \subset J}}\left(\frac{1}{\left|I_{T}\right|} \int\left|\phi_{j, k_{T}+\tilde{k}, n_{T}}^{*}(x) \psi_{j, k_{T}+\tilde{k}}^{*}(x)\left(f_{j} * \Phi_{j, k_{T}+\tilde{k}, T}\right)(x)\right|^{p_{j}-\delta} d x\right)^{\frac{p_{j}^{\prime}+\delta}{p_{j}-\delta}} 1_{I_{T}}(y)\right)^{\frac{p_{j}}{p_{j}^{\prime}+\delta}} d y \\
\leq C \lambda^{\varepsilon}|J|\left(\min \left\{2, \inf _{x \in J} M_{p_{j}}\left(M f_{j}\right)(x)\right\}\right)^{p_{j}} .
\end{array}
$$

By (4.23), we have

$$
\begin{array}{r}
\int\left(\sum_{\substack{T \in \mathcal{F}_{l} \\
I_{T} \subset J}}\left(\frac{1}{\left|I_{T}\right|} \int\left|\phi_{j, k_{T}+\tilde{k}, n_{T}}^{*}(x) \psi_{j, k_{T}+\tilde{k}}^{*}(x)\left(f_{j} * \Phi_{j, k_{T}+\tilde{k}, T}\right)(x)\right|^{p_{j}} d x\right)^{\frac{p_{j}^{\prime}+\delta}{p_{j}-\delta}} 1_{I_{T}}(y)\right)^{\frac{p_{j}}{p_{j}^{\prime}+\delta}} d y \\
\leq C \lambda^{\varepsilon}|J|\left(\min \left\{2 \inf _{x \in J} M_{p_{j}}\left(M f_{j}\right)(x)\right\}\right)^{p_{j}} .
\end{array}
$$

Using (3.10), we get

$$
\int\left(\sum_{\substack{T \in \mathcal{F}_{l} \\ I_{T} \subset J}} 1_{I_{T}}(y)\right)^{\frac{p_{j}}{p_{j}^{\prime}+\delta}} d y \leq C 2^{\frac{\eta p_{j}^{2} \mu}{p_{j}-\delta}} 2^{\frac{p_{j}^{2} \mu}{p_{j}^{\prime}\left(p_{j}-\delta\right)}} \lambda^{\varepsilon}|J|\left(\min \left\{2, \inf _{x \in J} M_{p_{j}}\left(M f_{j}\right)(x)\right\}\right)^{p_{j}}
$$


Therefore, we obtain

$$
\begin{aligned}
\left(\mathcal{N}_{\mathcal{F}_{l}}^{\frac{p_{j}}{p_{j}^{\prime}+\delta}}\right)^{\sharp}(x) & \leq \sup _{\substack{J \in\left\{I_{T}\right\}_{T \in \mathcal{F}_{l}} \\
x \in J}}\left(\frac{1}{|J|} \int\left(\sum_{\substack{T \in \mathcal{F}_{l} \\
I_{T} \subset J}} 1_{I_{T}}(y)\right)^{\frac{p_{j}}{p_{j}^{\prime}+\delta}} d y\right) \\
& \leq C 2^{\frac{\eta p_{j}^{2} \mu}{p_{j}-\delta}} 2^{\frac{p_{j}^{2} \mu}{p_{j}^{\prime}\left(p_{j}-\delta\right)}} \lambda^{\varepsilon}\left(\min \left\{2, M_{p_{j}}\left(M f_{j}\right)(x)\right\}\right)^{p_{j}} .
\end{aligned}
$$

Taking $L^{\frac{p_{j}^{\prime}+2 \delta}{p_{j}}}$ norms on both sides yields

$$
\left\|\left(\mathcal{N}_{\mathcal{F}_{l}}^{\frac{p_{j}}{p_{j}^{\prime}+\delta}}\right)^{\sharp}\right\|_{\frac{p_{j}^{\prime}+2 \delta}{p_{j}}} \leq C 2^{2 \eta p_{j} \mu} 2^{\frac{p_{j}^{2} \mu}{p_{j}^{\prime}\left(p_{j}-\delta\right)}} \lambda^{\varepsilon},
$$

since $\delta$ is very small. Thus, we obtain

$$
\int\left(\mathcal{N}_{\mathcal{F}_{l}}(x)\right)^{\frac{p_{j}^{\prime}+2 \delta}{p_{j}^{\prime}+\delta}} d x \leq C 2^{3 \eta p_{j}^{\prime} \mu} 2^{\frac{p_{j}\left(p_{j}^{\prime}+2 \delta\right) \mu}{p_{j}^{\prime}\left(p_{j}-\delta\right)}} \lambda^{\frac{\left(p_{j}^{\prime}+2 \delta\right) \varepsilon}{p_{j}}} .
$$

Thus, we obtain

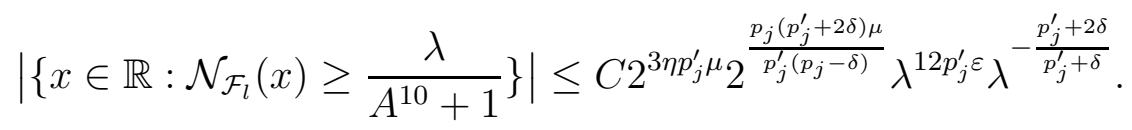

Therefore, we have

$$
\begin{aligned}
\mid\{x \in & \left.\mathbb{R}: \mathcal{N}_{\mathcal{F}^{\prime}}(x) \geq \lambda\right\} \mid \\
& \leq \sum_{l=1}^{A^{10}}\left|\left\{x \in \mathbb{R}: \mathcal{N}_{\mathcal{F}_{l}}(x) \geq \frac{\lambda}{A^{10}+1}\right\}\right|+\left|\left\{x \in \mathbb{R}: \mathcal{N}_{\mathcal{F}^{\prime \prime}}(x) \geq \frac{\lambda}{A^{10}+1}\right\}\right| \\
& \leq C 2^{4 \eta p_{j}^{\prime} \mu} 2^{\frac{p_{j}\left(p_{j}^{\prime}+2 \delta\right) \mu}{p_{j}^{\prime}\left(p_{j}-\delta\right)}} \lambda^{18 p_{j}^{\prime} \varepsilon} \lambda^{-\frac{p_{j}^{\prime}+2 \delta}{p_{j}^{\prime}+\delta}}+C \lambda^{10 \varepsilon-1}\left\|\mathcal{N}_{\mathcal{F}^{\prime \prime}}\right\|_{1} \\
& \leq C 2^{4 \eta p_{j}^{\prime} \mu} 2^{\frac{p_{j}\left(p_{j}^{\prime}+2 \delta\right) \mu}{p_{j}^{\prime}\left(p_{j}-\delta\right)}} \lambda^{18 p_{j}^{\prime} \varepsilon} \lambda^{-\frac{p_{j}^{\prime}+2 \delta}{p_{j}^{\prime}+\delta}}+C \lambda^{10 \varepsilon-1} e^{-A}\left\|\mathcal{N}_{\mathcal{F}_{1}}\right\|_{1} \\
& \leq C 2^{4 \eta p_{j}^{\prime} \mu} 2^{\frac{p_{j}\left(p_{j}^{\prime}+2 \delta\right) \mu}{p_{j}^{\prime}\left(p_{j}-\delta\right)}} \lambda^{18 p_{j}^{\prime} \varepsilon} \lambda^{-\frac{p_{j}^{\prime}+2 \delta}{p_{j}^{\prime}+\delta}}+C \lambda^{10 \varepsilon-1} e^{-A}\left\|\mathcal{N}_{\mathcal{F}_{1}}\right\|_{\frac{p_{j}^{\prime}+2 \delta}{p_{j}^{\prime}+\delta}}^{p_{j}^{\prime}+2 \delta} \\
& \leq C 2^{4 \eta p_{j}^{\prime} \mu} 2^{\frac{p_{j}\left(p_{j}^{\prime}+2 \delta\right) \mu}{p_{j}^{\prime}\left(p_{j}-\delta\right)}} \lambda^{18 p_{j}^{\prime} \varepsilon} \lambda^{-\frac{p_{j}^{\prime}+2 \delta}{p_{j}^{\prime}+\delta}}+C 2^{4 \eta p_{j} \mu} 2^{\frac{p_{j}\left(p_{j}^{\prime}+2 \delta\right) \mu}{p_{j}^{\prime}\left(p_{j}-\delta\right)}} \lambda^{18 p_{j}^{\prime} \varepsilon-1} e^{-\lambda^{\varepsilon}} \\
& \leq C 2^{4 \eta p_{j}^{\prime} \mu} 2^{\frac{p_{j}\left(p_{j}^{\prime}+2 \delta\right) \mu}{p_{j}^{\prime}\left(p_{j}-\delta\right)}} \lambda^{18 p_{j}^{\prime} \varepsilon} \lambda^{-\frac{p_{j}^{\prime}+2 \delta}{p_{j}^{\prime}+\delta}} \\
& \leq C 2^{10 \eta p_{j}^{\prime} \mu} 2^{\mu} \lambda^{18 p_{j}^{\prime} \varepsilon-\frac{p_{j}^{\prime}+2 \delta}{p_{j}^{\prime}+\delta}} \leq C 2^{10 \eta p_{j}^{\prime} \mu} 2^{\mu} \lambda^{-1-\varepsilon},
\end{aligned}
$$

where we chose $\varepsilon \ll \delta \ll \eta$. This completes the proof of (6.2). 


\section{Counting the trees, part II}

In this section, for $(i, j, \nu) \in \bigcup_{i, j \in\{2,3\}}\left(\{(1, j, 1),(i, 1,1)\} \bigcup_{\nu=3}^{4}\{(i, j, \nu)\}\right)$, we prove (3.20). We only prove the case $(1, j, 1)$ for $j \in\{2,3\}$. The proof of the other cases is similar. Let

$$
\mathcal{F}_{1, j, 1}=\bigcup_{l} T_{\mu, 1, j, l}^{1} \quad \text { and } \quad \mathcal{N}_{\mathcal{F}_{1, j, 1}}(x)=\sum_{T \in \mathcal{F}_{1, j, 1}} 1_{I_{T}}(x) .
$$

It is enough to prove that

$$
\left\|\mathcal{N}_{\mathcal{F}_{1, j, 1}}\right\|_{1} \leq C 2^{10 \eta p_{j}^{\prime} \mu} 2^{\mu} .
$$

But since $\mathcal{N}_{\mathcal{F}_{1, j, 1}}$ is integer-valued, it is sufficient to show that there exists $0<\varepsilon<\eta$ such that, for any $\lambda \geq 1$,

$$
\left|\left\{x \in \mathbb{R}: \mathcal{N}_{\mathcal{F}_{1, j, 1}}(x) \geq \lambda\right\}\right| \leq C_{\varepsilon} 2^{10 \eta p_{j}^{\prime} \mu} 2^{\mu} \lambda^{-1-\varepsilon} .
$$

As in [8], take $\mathcal{F}^{\prime} \subset \mathcal{F}_{1, j, 1}$ such that $\mathcal{N}_{\mathcal{F}^{\prime}}(x) \leq \lambda$ and

$$
\left|\left\{x \in \mathbb{R}: \mathcal{N}_{\mathcal{F}^{\prime}}(x) \geq \lambda\right\}\right|=\left|\left\{x \in \mathbb{R}: \mathcal{N}_{\mathcal{F}_{1, j, 1}}(x) \geq \lambda\right\}\right| .
$$

Recall the partial order $<$ defined on products of intervals in [8]. For $T \in \mathcal{F}^{\prime}$, define

$$
\begin{aligned}
T^{\text {min }} & =\left\{s \in T: I_{s} \times \omega_{1, s} \text { is minimal w.r.t. }<\right\} \\
T^{\partial} & =\left\{s \in T: I_{s} \cap\left(1-2^{-4}\right) I_{T}=\emptyset\right\}, \\
T^{\partial \max } & =\left\{s \in T^{\partial}: I_{s} \times \omega_{1, s} \text { is maximal in } T^{\partial} \text { w.r.t. }<\right\} \\
T^{\text {fat }} & =\left\{s \in T \backslash T^{\text {min }}: 2^{\eta \mu}\left|I_{s}\right| \geq\left|I_{T}\right|\right\}, \\
T^{\text {nice }} & =T \backslash\left(T^{\text {min }} \cup T^{\partial} \cup T^{\text {fat }}\right) .
\end{aligned}
$$

Note that by (3.10) (which fails at the step $\mu-1$ ) we have, for any $T \in \mathcal{F}^{\prime}$,

$$
\begin{aligned}
\| \sum_{(k, n) \in T^{\min }} \mid \phi_{j, k, n}^{*} \psi_{j, k}^{*} & \left.\left.\left(f_{j} * \Phi_{j, k, T}\right)\right|^{2}\right)^{\frac{1}{2}} \|_{p_{j}} \\
& \leq\left(\sum_{(k, n) \in T^{\min }}\left\|\phi_{j, k, n}^{*} \psi_{j, k}^{*}\left(f_{j} * \Phi_{j, k, T}\right)\right\|_{p_{j}}^{p_{j}}\right)^{\frac{1}{p_{j}}} \\
& \leq\left(\sum_{(k, n) \in T^{\min }} 2^{-\eta p_{j}(\mu-1)} 2^{-\frac{p_{j}(\mu-1)}{p_{j}^{\prime}}}\left|I_{k, n}\right|\right)^{\frac{1}{p_{j}}} \\
& \leq 2^{-\eta(\mu-1)} 2^{-\frac{(\mu-1)}{p_{j}^{\prime}}}\left|I_{T}\right|^{\frac{1}{p_{j}}} \leq 2^{2} 2^{-\frac{\mu}{p_{j}^{\prime}}}\left|I_{T}\right|^{\frac{1}{p_{j}}} .
\end{aligned}
$$


And also we have

$$
\begin{aligned}
\left\|\left(\sum_{(k, n) \in T^{\mathrm{fat}}}\left|\phi_{j, k, n}^{*} \psi_{j, k}^{*}\left(f_{j} * \Phi_{j, k, T}\right)\right|^{2}\right)^{\frac{1}{2}}\right\|_{p_{j}} \\
\leq\left(\sum_{(k, n) \in T^{\mathrm{fat}}}\left\|\phi_{j, k, n}^{*} \psi_{j, k}^{*}\left(f_{j} * \Phi_{j, k, T}\right)\right\|_{p_{j}}^{p_{j}}\right)^{\frac{1}{p_{j}}} \\
\leq\left(\sum_{(k, n) \in T^{\mathrm{fat}}} 2^{-\eta p_{j}(\mu-1)} 2^{-\frac{p_{j}(\mu-1)}{p_{j}^{\prime}}}\left|I_{k, n}\right|\right)^{\frac{1}{p_{j}}} \leq 2^{2} 2^{-\frac{\mu}{p_{j}^{\prime}}}\left|I_{T}\right|^{\frac{1}{p_{j}}} .
\end{aligned}
$$

Let $T_{t}^{\partial \max }$ be a tree of type 1 in $T^{\partial}$ with top $t \in T^{\partial \max }$. Then, by (3.8) we also have

$$
\begin{aligned}
\left\|\left(\sum_{(k, n) \in T^{\partial}}\left|\phi_{j, k, n}^{*} \psi_{j, k}^{*}\left(f_{j} * \Phi_{j, k, T}\right)\right|^{2}\right)^{\frac{1}{2}}\right\| \\
\leq\left(\sum_{t \in T^{\partial \max }}\left\|\left(\sum_{(k, n) \in T_{t}^{\partial \max }}\left|\phi_{j, k, n}^{*} \psi_{j, k}^{*}\left(f_{j} * \Phi_{j, k, T}\right)\right|^{2}\right)^{\frac{1}{2}}\right\|_{p_{j}}^{p_{j}}\right)^{\frac{1}{p_{j}}} \\
\leq\left(\sum_{t \in T^{\partial \max }} 2^{4 p_{j}} 2^{-\frac{p_{j}(\mu-1)}{p_{j}^{\prime}}}\left|I_{t}\right|\right)^{\frac{1}{p_{j}}} \leq 2^{2} 2^{-\frac{\mu}{p_{j}^{\prime}}}\left|I_{T}\right|^{\frac{1}{p_{j}}} .
\end{aligned}
$$

Therefore, by (3.8), we obtain

$$
\left|I_{T}\right|^{\frac{1}{p_{j}}} \leq C 2^{\frac{\mu}{p_{j}^{\prime}}}\left\|\left(\sum_{(k, n) \in T^{\text {nice }}}\left|\phi_{j, k, n}^{*} \psi_{j, k}^{*}\left(f_{j} * \Phi_{j, k, T}\right)\right|^{2}\right)^{\frac{1}{2}}\right\|_{p_{j}} .
$$

For $T \in \mathcal{F}^{\prime}, f \in \mathcal{S}, x \in \mathbb{R}, y \in \mathbb{R}$, and $(k, n) \in T^{\text {nice }}$, define

$$
B_{T, x, k, n} f(y)=\phi_{j, k, n}^{*}(x)\left(f * \Phi_{j, k, T}\right)(x) 1_{I_{T}}(y)
$$

and we let $\vec{B} f=\left\{B_{T, x, k, n} f\right\}_{T, x, k, n}$ be the corresponding vector-valued operator. Let $A=\lambda^{\varepsilon}$. Then by the almost orthogonality lemma in [8], we obtain

$$
\begin{aligned}
& \|\vec{B} f\|_{L^{2}\left(\mathbb{R}, l^{2}\left(\mathcal{F}^{\prime}, L^{2}\left(I_{T}, l^{2}\left(T^{\text {nice })}\right)\right)\right)\right.} \\
& \leq\left(\int \sum_{T \in \mathcal{F}^{\prime}} \frac{1}{\left|I_{T}\right|} \int \sum_{(k, n) \in T^{\text {nice }}}\left|\phi_{j, k, n}^{*}(x)\left(f * \Phi_{j, k, T}\right)(x)\right|^{2} \varphi_{T}(x) d x 1_{I_{T}}(y) d y\right)^{\frac{1}{2}} \\
& \leq\left(\sum_{T \in \mathcal{F}^{\prime}} \sum_{(k, n) \in T^{\text {nice }}}\left\|\phi_{j, k, n}^{*}\left(f * \Phi_{j, k, T}\right)\right\|_{2}^{2}\right)^{\frac{1}{2}} \leq C\left(1+A^{-\frac{1}{\varepsilon}} \lambda\right)\|f\|_{2} \leq C\|f\|_{2} .
\end{aligned}
$$


On the other hand, by (4.5), we also have

$$
\begin{aligned}
& \|\vec{B} f\|_{L^{1+2 \delta}\left(\mathbb{R}, l^{\infty}\left(\mathcal{F}^{\prime}, L^{1+\delta}\left(I_{T}, l^{2}\left(T^{\text {nice }}\right)\right)\right)\right)} \\
& \leq\left(\int\left(\sup _{T \in \mathcal{F}^{\prime}} \frac{1}{\left|I_{T}\right|^{\frac{1}{1+\delta}}}\left\|\left(\sum_{(k, n) \in T^{\text {nice }}}\left|\phi_{j, k, n}^{*}\left(f * \Phi_{j, k, T}\right)\right|^{2}\right)^{\frac{1}{2}}\right\| \|_{1+\delta} 1_{I_{T}}(y)\right)^{1+2 \delta} d y\right)^{\frac{1}{1+2 \delta}} \\
& \leq\left(\int\left(M_{1+\delta} f(y)\right)^{1+2 \delta} d y\right)^{\frac{1}{1+2 \delta}} \leq C\|f\|_{1+2 \delta}
\end{aligned}
$$

where $\delta>0$ is a very small number.

Therefore, by complex interpolation and the fact that $L^{q}\left(I_{T}\right) \subset L^{p}\left(I_{T}\right)$ for $q \geq p$, we obtain

$$
\|\vec{B} f\|_{L^{p_{j}}\left(\mathbb{R}, l^{p_{j}^{\prime}+\delta}\left(\mathcal{F}^{\prime}, L^{p_{j}-\delta}\left(I_{T}, l^{2}\left(T^{\text {nice }}\right)\right)\right)\right)} \leq C\|f\|_{p_{j}}
$$

Note that $\left|\phi_{j, k, n}^{*}(x)\right| \leq C \varphi_{T}(x)$, we have

$$
\begin{gathered}
\int\left(\sum_{T \in \mathcal{F}^{\prime}}\left(\frac{1}{\left|I_{T}\right|} \int\left(\sum_{(k, n) \in T^{\mathrm{nice}}}\left|\phi_{j, k, n}^{*}(x)\left(f * \Phi_{j, k, T}\right)(x)\right|^{2}\right)^{\frac{p_{j}-\delta}{2}} d x\right)^{\frac{p_{j}^{\prime}+\delta}{p_{j}-\delta}} 1_{I_{T}}(y)\right)^{\frac{p_{j}}{p_{j}^{\prime}+\delta}} d y \\
\leq C\|f\|_{p_{j}}^{p_{j}}
\end{gathered}
$$

As in [8] and [12], we use a localization argument to obtain a local estimate related to (7.4). In fact, $H_{J}(f)$ be

$$
\int\left(\sum_{\substack{T \in \mathcal{F}^{\prime} \\ I_{T} \subset J}}\left(\frac{1}{\left|I_{T}\right|} \int\left(\sum_{(k, n) \in T^{\mathrm{nice}}}\left|\phi_{j, k, n}^{*}(x)\left(f * \Phi_{j, k, T}\right)(x)\right|^{2}\right)^{\frac{p_{j}-\delta}{2}} d x\right)^{\frac{p_{j}^{\prime}+\delta}{p_{j}-\delta}} 1_{I_{T}}(y)\right)^{\frac{p_{j}}{p_{j}^{\prime}+\delta}} d y
$$

Then we have

$$
H_{J}(f) \leq H_{J}\left(f 1_{2 \lambda^{\varepsilon} J}\right)+H_{J}\left(f 1_{\left(2 \lambda^{\varepsilon} J\right)^{c}}\right)
$$

where $J \in\left\{I_{T}\right\}_{T \in \mathcal{F}^{\prime}}$. By (7.4), we have

$$
H_{J}\left(f 1_{2 \lambda^{\varepsilon} J}\right) \leq C\left\|f 1_{2 \lambda^{\varepsilon} J}\right\|_{p_{j}}^{p_{j}} \leq C \lambda^{\varepsilon}|J|\left(\inf _{x \in J} M_{p_{j}}(M f)(x)\right)^{p_{j}} .
$$


And $H_{J}\left(f 1_{\left(2 \lambda^{\varepsilon} J\right)^{c}}\right)$ is estimated by

$$
\begin{aligned}
& \int\left\{\sum _ { \substack { T \in \mathcal { F } ^ { \prime } \\
I _ { T } \subset J } } \left[\frac { 1 } { | I _ { T } | } \int \left(\sum_{(k, n) \in T^{\text {nice }}} \frac{1}{\left(1+2^{-k+m} \operatorname{dist}\left(x, I_{k, n}\right)\right)^{2 N}}\right.\right.\right. \\
& \left.\left.\cdot\left|\int_{\left(2 \lambda^{\varepsilon} J\right)^{c}} \frac{|f(z)| 2^{-k+m} d z}{\left(1+2^{-k+m}|x-z|\right)^{N}}\right|^{2}\right)\left.^{\frac{p_{j}-\delta}{2}} d x\right|^{\frac{p_{j}^{\prime}+\delta}{p_{j}-\delta}} 1_{I_{T}}(y)\right\}^{\frac{p_{j}}{p_{j}^{\prime}+\delta}} d y \\
& \leq \int\left(\sum_{\substack{T \in \mathcal{F}^{\prime} \\
I_{T} \subset J}}\left(\frac{1}{\left|I_{T}\right|} \sum_{(k, n) \in T^{\text {nice }}} \frac{\left(\inf _{x \in I_{T}} M f(x)\right)^{p_{j}-\delta}\left|I_{k, n}\right|}{\lambda^{p_{j}-\delta}\left(1+2^{-k} \operatorname{dist}\left(I_{k, n},\left(2 I_{T}\right)^{c}\right)\right)^{N}}\right)^{\frac{p_{j}^{\prime}+\delta}{p_{j}-\delta}} 1_{I_{T}}(y)\right)^{\frac{p_{j}}{p_{j}^{\prime}+\delta}} d y \\
& \leq \int\left(\sum_{\substack{T \in \mathcal{F}^{\prime} \\
I_{T} \subset J}}\left(\inf _{x \in I_{T}} M f(x)\right)^{p_{j}^{\prime}+\delta} \lambda^{-\frac{p_{j}^{\prime}+\delta}{p_{j}-\delta}} 1_{I_{T}}(y)\right)^{\frac{p_{j}}{p_{j}^{\prime}+\delta}} d y \\
& \leq \frac{C}{\lambda} \int \sum_{\substack{T \in \mathcal{F}_{l} \\
I_{T} \subset J}}\left(\inf _{x \in I_{T}} M f(x)\right)^{p_{j}} 1_{I_{T}}(y) d y \leq C|J|\left(\inf _{x \in J} M_{p_{j}}(M f)(x)\right)^{p_{j}} .
\end{aligned}
$$

Hence, we obtain $H_{J}(f) \leq C \lambda^{\varepsilon}|J|\left(\inf _{x \in J} M_{p_{j}}(M f)(x)\right)^{p_{j}}$. Notice that $\phi_{j, k, n}^{*}(x) \psi_{j, k}^{*}(x) \leq\left(1+2^{-k_{T}+m} \operatorname{dist}\left(J, E^{c}\right)\right)^{-N}$. As we proved (4.6), we may sharpen the previous estimate to

$$
\begin{aligned}
& \int\left(\sum_{\substack{T \in \mathcal{F}^{\prime} \\
I_{T} \subset J}}\left(\frac{1}{\left|I_{T}\right|} \int\left(\sum_{(k, n) \in T^{\text {nice }}}\left|\phi_{j, k, n}^{*}(x) \psi_{j, k}^{*}(x)\left(f_{j} * \Phi_{j, k, T}\right)(x)\right|^{2}\right)^{\frac{p_{j}-\delta}{2}} d x\right)^{\frac{p_{j}^{\prime}+\delta}{p_{j}-\delta}} 1_{I_{T}}(y)\right)^{\frac{p_{j}}{p_{j}^{\prime}+\delta}} d y \\
& \quad \leq C \lambda^{\varepsilon}|J|\left(\min \left\{2 \inf _{x \in J} M_{p_{j}}\left(M f_{j}\right)(x)\right\}\right)^{p_{j}} .
\end{aligned}
$$

Notice that

$$
\left\|\left(\sum_{(k, n) \in T^{\text {nice }}}\left|\phi_{j, k, n}^{*} \psi_{j, k}^{*}\left(f_{j} * \Phi_{j, k, T}\right)\right|^{2}\right)^{\frac{1}{2}}\right\|_{B M O} \leq C .
$$

The proof of this inequality is similar as the proof of (4.24). Thus, we have

$$
\begin{aligned}
& \int\left(\sum_{\substack{T \in \mathcal{F}^{\prime} \\
I_{T} \subset J}}\left(\frac{1}{\left|I_{T}\right|} \int\left(\sum_{(k, n) \in T^{\text {nice }}}\left|\phi_{j, k, n}^{*}(x) \psi_{j, k}^{*}(x)\left(f_{j} * \Phi_{j, k, T}\right)(x)\right|^{2}\right)^{\frac{p_{j}}{2}} d x\right)^{\frac{p_{j}^{\prime}+\delta}{p_{j}-\delta}} 1_{I_{T}}(y)\right)^{\frac{p_{j}}{p_{j}^{\prime}+\delta}} d y \\
& \quad \leq C \lambda^{\varepsilon}|J|\left(\min \left\{2 \inf _{x \in J} M_{p_{j}}\left(M f_{j}\right)(x)\right\}\right)^{p_{j}} .
\end{aligned}
$$


Using (7.2), we obtain

$$
\int\left(\sum_{\substack{T \in \mathcal{F}^{\prime} \\ I_{T} \subset J}} 1_{I_{T}}(y)\right)^{\frac{p_{j}}{p_{j}^{\prime}+\delta}} d y \leq C 2^{\frac{p_{j}^{2} \mu}{p_{j}^{\prime}\left(p_{j}-\delta\right)}} \lambda^{\varepsilon}|J|\left(\min \left\{2, \inf _{x \in J} M_{p_{j}}\left(M f_{j}\right)(x)\right\}\right)^{p_{j}}
$$

Therefore, we have

$$
\begin{aligned}
\left(\mathcal{N}_{\mathcal{F}^{\prime}}^{\frac{p_{j}}{p_{j}^{\prime}+\delta}}\right)^{\sharp}(x) & \leq \sup _{\substack{J \in\left\{I_{T}\right\}_{T \in \mathcal{F}^{\prime}} \\
x \in J}}\left(\frac{1}{|J|} \int\left(\sum_{\substack{T \in \mathcal{F}^{\prime} \\
I_{T} \subset J}} 1_{I_{T}}(y)\right)^{\frac{p_{j}}{p_{j}^{\prime}+\delta}} d y\right) \\
& \leq C 2^{\frac{p_{j}^{2} \mu}{p_{j}^{\prime}\left(p_{j}-\delta\right)}} \lambda^{\varepsilon}\left(\min \left\{2, M_{p_{j}}\left(M f_{j}\right)(x)\right\}\right)^{p_{j}} .
\end{aligned}
$$

Taking $L^{\frac{p_{j}^{\prime}+2 \delta}{p_{j}}}$ norms on both sides yields

$$
\left\|\left(\mathcal{N}_{\mathcal{F}^{\prime}}^{\frac{p_{j}}{p_{j}^{\prime}+\delta}}\right)^{\sharp}\right\|_{\frac{p_{j}^{\prime}+2 \delta}{p_{j}}} \leq C 2^{2 \eta p_{j} \mu} 2^{\frac{p_{j}^{2} \mu}{p_{j}^{\prime}\left(p_{j}-\delta\right)}} \lambda^{\varepsilon}
$$

since $\delta$ is very small. Thus, we obtain

$$
\int\left(\mathcal{N}_{\mathcal{F}^{\prime}}(x)\right)^{\frac{p_{j}^{\prime}+2 \delta}{p_{j}^{\prime}+\delta}} d x \leq C 2^{3 \eta p_{j}^{\prime} \mu} 2^{\frac{p_{j}\left(p_{j}^{\prime}+2 \delta\right) \mu}{p_{j}^{\prime}\left(p_{j}-\delta\right)}} \lambda^{\frac{\left(p_{j}^{\prime}+2 \delta\right) \varepsilon}{p_{j}}} .
$$

Therefore, we have proved that

$$
\left|\left\{x \in \mathbb{R}: \mathcal{N}_{\mathcal{F}^{\prime}}(x) \geq \lambda\right\}\right| \leq C 2^{3 \eta p_{j}^{\prime} \mu} 2^{\frac{p_{j}\left(p_{j}^{\prime}+2 \delta\right) \mu}{p_{j}^{\prime}\left(p_{j}-\delta\right)}} \lambda^{2 p_{j}^{\prime} \varepsilon} \lambda^{-\frac{p_{j}^{\prime}+2 \delta}{p_{j}^{\prime}+\delta}}
$$

Choose $\varepsilon \ll \delta \ll \eta$, then the estimate above implies (7.1).

\section{Counting the trees, part III}

In this section, we prove (3.20) for $i, j \in\{2,3\}$ and $\nu=5$. Let $\mathcal{F}_{i, j, 5}=$ $\bigcup_{l} T_{\mu, i, j, l}^{5}$ and $\mathcal{N}_{\mathcal{F}_{i, j, 5}}(x)=\sum_{T \in \mathcal{F}_{i, j, 5}} 1_{I_{T}}(x)$. It is sufficient to prove

$$
\left\|\mathcal{N}_{\mathcal{F}_{i, j, 5}}\right\|_{1} \leq C 2^{10 \eta p_{i}^{\prime} \mu} 2^{\mu} .
$$

Notice that $\mathcal{N}_{\mathcal{F}_{i, j, 5}}$ is integer-valued, to prove (8.1), it suffices to show that there exists $0<\varepsilon<\eta$ such that, for any $\lambda \geq 1$,

$$
\left|\left\{x \in \mathbb{R}: \mathcal{N}_{\mathcal{F}_{i, j, 5}}(x) \geq \lambda\right\}\right| \leq C_{\varepsilon} 2^{10 \eta p_{i}^{\prime} \mu} 2^{\mu} \lambda^{-1-\varepsilon} .
$$


As in [8], take $\mathcal{F}^{\prime} \subset \mathcal{F}_{i, j, 5}$ such that $\mathcal{N}_{\mathcal{F}^{\prime}}(x) \leq \lambda$ and

$$
\left|\left\{x \in \mathbb{R}: \mathcal{N}_{\mathcal{F}^{\prime}}(x) \geq \lambda\right\}\right|=\left|\left\{x \in \mathbb{R}: \mathcal{N}_{\mathcal{F}_{i, j, 5}}(x) \geq \lambda\right\}\right| .
$$

Assume that (3.15) holds for $\tilde{k}=L$ (other cases are similar). Let $Q=$ $\left\{(k, J, T): k \in \mathbb{Z}_{r}, J \in \Delta_{k-m, T}, T \in \mathcal{F}^{\prime}\right\}$ and $A=\lambda^{\varepsilon}$. We only prove the case when $\lambda \geq 2^{\eta \mu}$. The case $\lambda \leq 2^{\eta \mu}$ can be proved by the same method. As in [8], using the separation lemma in [11], we have

$$
Q=\left(\bigcup_{l=1}^{A^{10}} Q_{l}\right) \bigcup Q^{\prime},
$$

such that

$$
\left(A J \times \omega_{j, k+L, T}\right) \cap\left(A J^{\prime} \times \omega_{j, k^{\prime}+L, T^{\prime}}\right)=\emptyset,
$$

for $1 \leq l \leq A^{10}, q \neq q^{\prime}$ and $q, q^{\prime} \in Q_{l}$, where $q=(k, J, T)$ and $q^{\prime}=\left(k^{\prime}, J^{\prime}, T^{\prime}\right)$. And

$$
\sum_{\substack{q \in Q^{\prime} \\ q=(k, J, T)}}|J| \leq C e^{-A} \sum_{\substack{q \in Q_{1} \\ q=(k, J, T)}}|J| .
$$

For $1 \leq l \leq A^{10}$ and $T \in \mathcal{F}^{\prime}$, let

$$
Q_{l, T}=\left\{(k, J): k \in \mathbb{Z}_{r}, J \in \Delta_{k-m, T},(k, J, T) \in Q_{l}\right\} .
$$

For $(k, J) \in Q_{l, T}, f \in \mathcal{S}$, define

$$
B_{T, x, k, J} f(y)=\rho_{k-m, J}(x)\left(f * \Phi_{j, k+L, T}\right)(x) 1_{I_{T}}(y),
$$

and let $\vec{B}$ be the corresponding vector-valued operator. Then by the almost orthogonality lemma in [8], we obtain

$$
\begin{aligned}
\| & \vec{B} f \|_{L^{2}\left(\mathbb{R}, l^{2}\left(\mathcal{F}^{\prime}, L^{2}\left(I_{T}, l^{2}\left(Q_{l, T}\right)\right)\right)\right.} \\
& \leq\left(\int \sum_{T \in \mathcal{F}^{\prime}} \frac{1}{\left|I_{T}\right|} \int_{(k, J) \in Q_{l, T}}\left|\rho_{k-m, J}(x)\left(f * \Phi_{j, k+L, T}\right)(x)\right|^{2} \varphi_{T}(x) d x 1_{I_{T}}(y) d y\right)^{\frac{1}{2}} \\
& \leq\left(\sum_{T \in \mathcal{F}^{\prime}} \sum_{(k, J) \in Q_{l, T}}\left\|\rho_{k-m, J}\left(f * \Phi_{j, k+L, T}\right)\right\|_{2}^{2}\right)^{\frac{1}{2}} \\
& \leq\left(\sum_{\substack{q \in Q_{l} \\
q=(k, J, T)}}\left\|\rho_{k-m, J}\left(f * \Phi_{j, k+L, T}\right)\right\|_{2}^{2}\right)^{\frac{1}{2}} \\
& \leq C\left(1+A^{-\frac{1}{\varepsilon}} \lambda\right)\|f\|_{2} \leq C\|f\|_{2} .
\end{aligned}
$$


On the other hand, by $(4.20)$, we get $\|\vec{B} f\|_{L^{1+2 \delta}\left(\mathbb{R}, l^{\infty}\left(\mathcal{F}^{\prime}, L^{1+\delta}\left(I_{T}, l^{2}\left(Q_{l, T}\right)\right)\right)\right.}$ is dominated by

$$
\left.\left(\int \sup _{T \in \mathcal{F}^{\prime}}\left(\frac{1}{\left|I_{T}\right|} \int\left(\sum_{(k, J) \in Q_{l, T}}\left|\rho_{k-m, J}(x)\left(f * \Phi_{j, k+L, T}\right)(x)\right|^{2}\right)^{\frac{1+\delta}{2}} \varphi_{T}(x) d x\right)^{\frac{1}{1+\delta}} 1_{I_{T}}(y)\right)^{1+2 \delta} d y\right)^{\frac{1}{1+2 \delta}}
$$

which is controlled by

$$
\left(\int\left(\left.\sup _{T \in \mathcal{F}^{\prime}} \frac{1}{\left|I_{T}\right|^{\frac{1}{1+\delta}}}\left\|\left(\sum_{(k, J) \in Q_{l, T}}\left|\rho_{k-m, J}\left(f * \Phi_{j, k+L, T}\right)\right|^{2}\right)^{\frac{1}{2}}\right\|\right|_{1+\delta} 1_{I_{T}}(y)\right)^{1+2 \delta} d y\right)^{\frac{1}{1+2 \delta}} .
$$

And this term is clearly dominated by

$$
\left(\int\left(M_{1+\delta} f(y)\right)^{1+2 \delta} d y\right)^{\frac{1}{1+2 \delta}} \leq C\|f\|_{1+2 \delta},
$$

where $\delta>0$ is a very small number.

Therefore, by complex interpolation and the fact that $L^{q}\left(I_{T}\right) \subset L^{p}\left(I_{T}\right)$ for $q \geq p$, we obtain

$$
\|\vec{B} f\|_{L^{p_{j}}\left(\mathbb{R}, l^{p_{j}^{\prime}+\delta}\left(\mathcal{F}^{\prime}, L^{p_{j}-\delta}\left(I_{T}, l^{2}\left(Q_{l, T}\right)\right)\right)\right)} \leq C\|f\|_{p_{j}} .
$$

Define $K(f)$ to be

$$
\int\left(\sum_{T \in \mathcal{F}^{\prime}}\left(\frac{1}{\left|I_{T}\right|} \int\left(\sum_{(k, J) \in Q_{l, T}}\left|\rho_{k-m, J}(x)\left(f * \Phi_{j, k+L, T}\right)(x)\right|^{2}\right)^{\frac{p_{j}-\delta}{2}} d x\right)^{\frac{p_{j}^{\prime}+\delta}{p_{j}-\delta}} 1_{I_{T}}(y)\right)^{\frac{p_{j}}{p_{j}^{\prime}+\delta}} d y .
$$

Note that $\left|\rho_{k-m, J}(x)\right| \leq C \varphi_{T}(x)$, we have

$$
|K(f)| \leq C\|f\|_{p_{j}}^{p_{j}}
$$

As in [8] and [12], we use a localization argument to obtain a local estimate related to (8.6). In fact, let $K_{I}(f)$ be

$$
\int\left(\sum_{\substack{T \in \mathcal{F}^{\prime} \\ I_{T} \subset I}}\left(\frac{1}{\left|I_{T}\right|} \int\left(\sum_{(k, J) \in Q_{l, T}}\left|\rho_{k-m, J}(x)\left(f * \Phi_{j, k+L, T}\right)(x)\right|^{2}\right)^{\frac{p_{j}-\delta}{2}} d x\right)^{\frac{p_{j}^{\prime}+\delta}{p_{j}-\delta}} 1_{I_{T}}(y)\right)^{\frac{p_{j}}{p_{j}^{\prime}+\delta}} d y
$$

where $I \in\left\{I_{T}\right\}_{T \in \mathcal{F}^{\prime}}$. Then we have $K_{I}(f) \leq K_{I}\left(f 1_{2 \lambda^{\varepsilon} I}\right)+K_{I}\left(f 1_{\left(2 \lambda^{\varepsilon} I\right)^{c}}\right)$. 
By (8.6), we have

$$
K_{I}\left(f 1_{2 \lambda^{\varepsilon} I}\right) \leq C\left\|f 1_{2 \lambda^{\varepsilon} I}\right\|_{p_{j}}^{p_{j}} \leq C \lambda^{\varepsilon}|I|\left(\inf _{x \in I} M_{p_{j}}(M f)(x)\right)^{p_{j}} .
$$

And $K_{I}\left(f 1_{\left(2 \lambda^{\varepsilon} I\right)^{c}}\right)$ is estimated by

$$
\begin{aligned}
& \int\left\{\sum _ { \substack { T \in \mathcal { F } ^ { \prime } \\
I _ { T } \subset I } } \left[\frac { 1 } { | I _ { T } | } \int \left(\sum_{(k, J) \in Q_{l, T}} \frac{1}{\left(1+|J|^{-1} \operatorname{dist}(x, J)\right)^{2 N}}\right.\right.\right. \\
& \left.\left.\cdot\left|\int_{\left(2 \lambda^{\varepsilon} J\right)^{c}} \frac{|f(z)| 2^{-k+m} d z}{\left(1+|J|^{-1}|x-z|\right)^{N}}\right|^{2}\right)\left.^{\frac{p_{j}-\delta}{2}} d x\right|^{\frac{p_{j}^{\prime}+\delta}{p_{j}-\delta}} 1_{I_{T}}(y)\right\}^{\frac{p_{j}}{p_{j}^{\prime}+\delta}} d y \\
& \leq \int\left(\sum_{\substack{T \in \mathcal{F}^{\prime} \\
I_{T} \subset I}}\left(\frac{1}{\left|I_{T}\right|} \int\left(\sum_{(k, J) \in Q_{l, T}} \frac{\left(\inf _{x \in I_{T}} M f(x)\right)^{2}}{\lambda^{2}\left(1+|J|^{-1} \operatorname{dist}(x, J)\right)^{N}}\right)^{\frac{p_{j}-\delta}{2}} d x\right)^{\frac{p_{j}^{\prime}+\delta}{p_{j}-\delta}} 1_{I_{T}}(y)\right)^{\frac{p_{j}}{p_{j}^{\prime}+\delta}} d y \\
& \leq \int\left(\sum_{\substack{T \in \mathcal{F}^{\prime} \\
I_{T} \subset I}}\left(\frac{1}{\left|I_{T}\right|} \sum_{(k, J) \in Q_{l, T}} \frac{\left(\inf _{x \in I_{T}} M f(x)\right)^{p_{j}-\delta}|J|}{\lambda^{p_{j}-\delta}}\right)^{\frac{p_{j}^{\prime}+\delta}{p_{j}-\delta}} 1_{I_{T}}(y)\right)^{\frac{p_{j}}{p_{j}^{\prime}+\delta}} d y \\
& \leq \int\left(\sum_{\substack{T \in \mathcal{F}^{\prime} \\
I_{T} \subset I}}\left(\inf _{x \in I_{T}} M f(x)\right)^{p_{j}^{\prime}+\delta} \lambda^{-\left(p_{j}^{\prime}+\delta\right)} 1_{I_{T}}(y)\right)^{\frac{p_{j}}{p_{j}^{\prime}+\delta}} d y \\
& \leq \frac{C}{\lambda} \int \sum_{\substack{T \in \mathcal{F}_{l} \\
I_{T} \subset I}}\left(\inf _{x \in I_{T}} M f(x)\right)^{p_{j}} 1_{I_{T}}(y) d y \leq C|I|\left(\inf _{x \in I} M_{p_{j}}(M f)(x)\right)^{p_{j}} .
\end{aligned}
$$

Hence, we obtain

$$
K_{I}(f) \leq C \lambda^{\varepsilon}|I|\left(\inf _{x \in I} M_{p_{j}}(M f)(x)\right)^{p_{j}} .
$$

Notice that

$$
\rho_{k-m, J}(x) \leq \frac{C}{\left(1+|J|^{-1} \operatorname{dist}(x, J)\right)^{N}\left(1+|J|^{-1} \operatorname{dist}\left(J, E^{c}\right)\right)^{N}} .
$$

As we proved (4.6), we may sharpen the previous estimate to

$$
K_{I}(f) \leq C \lambda^{\varepsilon}|I|\left(\min \left\{2, \inf _{x \in I} M_{p_{j}}\left(M f_{j}\right)(x)\right\}\right)^{p_{j}} .
$$

Also note that

$$
\left\|\left(\sum_{(k, J) \in Q_{l, T}}\left|\rho_{k-m, J}\left(f_{j} * \Phi_{j, k+L, T}\right)\right|^{2}\right)^{\frac{1}{2}}\right\|_{B M O} \leq C .
$$


The proof of this inequality is similar as the proof of (4.24). Let $K_{I}^{\prime}(f)$ be

$$
\int\left(\sum_{\substack{T \in \mathcal{F}^{\prime} \\ I_{T} \subset I}}\left(\frac{1}{\left|I_{T}\right|} \int\left(\sum_{(k, J) \in Q_{l, T}}\left|\rho_{k-m, J}(x)\left(f_{j} * \Phi_{j, k+L, T}\right)(x)\right|^{2}\right)^{\frac{p_{j}}{2}} d x\right)^{\frac{p_{j}^{\prime}+\delta}{p_{j}-\delta}} 1_{I_{T}}(y)\right)^{\frac{p_{j}}{p_{j}^{\prime}+\delta}} d y
$$

Thus, we have

$$
K_{I}^{\prime}(f) \leq C \lambda^{\varepsilon}|I|\left(\min \left\{2, \inf _{x \in I} M_{p_{j}}\left(M f_{j}\right)(x)\right\}\right)^{p_{j}} .
$$

Let $K_{I, Q}^{\prime \prime}(f)$ be

$$
\int\left(\sum_{\substack{T \in \mathcal{F}^{\prime} \\ I_{T} \subset I}}\left(\frac{1}{\left|I_{T}\right|} \int\left(\sum_{(k, J) \in Q}\left|\rho_{k-m, J}(x)\left(f_{j} * \Phi_{j, k+L, T}\right)(x)\right|^{2}\right)^{\frac{p_{j}}{2}} d x\right)^{\frac{p_{j}^{\prime}+\delta}{p_{j}-\delta}} 1_{I_{T}}(y)\right)^{\frac{p_{j}-\delta}{p_{j}^{\prime}+\delta}} d y
$$

Thus, by Hölder 's inequality, we obtain

$$
\begin{aligned}
K_{I, Q_{l, T}}^{\prime \prime}(f) & \leq\left(K_{I}^{\prime}(f)\right)^{\frac{p_{j}-\delta}{p_{j}}}|I|^{\frac{\delta}{p_{j}}} \\
& \leq C \lambda^{\varepsilon}|I|\left(\min \left\{2, \inf _{x \in I} M_{p_{j}}\left(M f_{j}\right)(x)\right\}\right)^{p_{j}-\delta} .
\end{aligned}
$$

Let

$$
Q_{T}=\left\{(k, J): k \in \mathbb{Z}_{r}, J \in \Delta_{k-m, T},(k, J, T) \in Q\right\}
$$

and

$$
Q_{T}^{\prime}=\left\{(k, J): k \in \mathbb{Z}_{r}, J \in \Delta_{k-m, T},(k, J, T) \in Q^{\prime}\right\} .
$$

Then we have

$$
K_{I, Q_{T}}^{\prime \prime}(f) \leq C \sum_{l=1}^{A^{10}} K_{I, Q_{l . T}}^{\prime \prime}(f)+C K_{I, Q_{T}^{\prime}}^{\prime \prime} .
$$

Here we used Minkowski's inequality in the last estimate. Using a previous estimate we obtain

$$
C \sum_{l=1}^{A^{10}} K_{I, Q_{l . T}}^{\prime \prime}(f) \leq C \lambda^{11 \varepsilon}|I|\left(\min \left\{2, \inf _{x \in I} M_{p_{j}}\left(M f_{j}\right)(x)\right\}\right)^{p_{j}-\delta}
$$

It remains to control $K_{I, Q_{T}^{\prime}}^{\prime}$. We have

$$
K_{I, Q_{T}^{\prime}}^{\prime \prime} \leq \int \sum_{\substack{T \in \mathcal{F}^{\prime} \\ I_{T} \subset I}}\left(\sum_{(k, J) \in Q_{T}^{\prime}}\left|\rho_{k-m, J}(x)\left(f_{j} * \Phi_{j, k+L, T}\right)(x)\right|^{2}\right)^{\frac{p_{j}}{2}} d x
$$




$$
\begin{aligned}
& \leq \sum_{\substack{T \in \mathcal{F}^{\prime} \\
I_{T} \subset I}} \sum_{(k, J) \in Q_{T}^{\prime}}\left\|\rho_{k-m, J}\left(f_{j} * \Phi_{j, k+L, T}\right)\right\|_{p_{j}}^{p_{j}} \\
& \leq C \sum_{\substack{q \in Q^{\prime} \\
q=(k, J, T) \\
I_{T} \subset I}}|J| \leq C e^{-A} \sum_{\substack{q \in Q_{1} \\
q=(k, J, T) \\
I_{T} \subset I}}|J| \\
& =C e^{-A} \sum_{\substack{T \in \mathcal{F}^{\prime} \\
I_{T} \subset I}} \sum_{J \in \Delta_{k-m, T}}|J| \leq C e^{-A} \sum_{\substack{T \in \mathcal{F}^{\prime} \\
I_{T} \subset I}}\left|I_{T}\right| \leq C e^{-A} \lambda|I| .
\end{aligned}
$$

We have now proved that

$$
K_{I, Q_{T}^{\prime}}^{\prime \prime} \leq C e^{-A} \lambda|I| .
$$

By (3.15), (8.7) and (8.8), we obtain

$$
\begin{aligned}
& \left\|\sum_{\substack{T \in \mathcal{F}^{\prime} \\
I_{T} \subset I}} 1_{I_{T}}\right\| \|_{\frac{p_{j}-\delta}{p_{j}^{\prime}+\delta}}^{\frac{p_{j}-\delta}{p_{j}^{\prime}+\delta}} \\
& \quad \leq C 2^{\frac{p_{j} \mu}{p_{j}^{\prime}}} \lambda^{11 \varepsilon}|I|\left(\min \left\{2, \inf _{x \in I} M_{p_{j}}\left(M f_{j}\right)(x)\right\}\right)^{p_{j}-\delta}+C 2^{\frac{p_{j} \mu}{p_{j}^{\prime}}} \frac{\lambda}{e^{A}}|I| .
\end{aligned}
$$

Therefore, we have

$$
\left(\mathcal{N}_{\mathcal{F}^{\prime}}^{\frac{p_{j}-\delta}{p_{j}^{\prime}+\delta}}\right)^{\sharp}(x) \leq C 2^{\frac{p_{j} \mu}{p_{j}^{\prime}}} \lambda^{11 \varepsilon}\left(\min \left\{2, M_{p_{j}}\left(M f_{j}\right)(x)\right\}\right)^{p_{j}-\delta}+C 2^{\frac{p_{j} \mu}{p_{j}^{\prime}}} e^{-A} \lambda
$$

Taking $L^{\frac{p_{j}^{\prime}+2 \delta}{p_{j}-\delta}}$ norms on both sides, we obtain

$$
\left\|\left(\mathcal{N}_{\mathcal{F}^{\prime}}^{\frac{p_{j}-\delta}{p_{j}^{\prime}+\delta}}\right)^{\sharp}\right\|_{\frac{p_{j}^{\prime}+2 \delta}{p_{j}-\delta}} \leq C 2^{\frac{p_{j} \mu}{p_{j}^{\prime}}} \lambda^{12 \varepsilon}+C 2^{\frac{p_{j} \mu}{p_{j}^{\prime}}} e^{-A} \lambda\left|\bigcup_{T \in \mathcal{F}^{\prime}} I_{T}\right|^{\frac{p_{j}-\delta}{p_{j}^{\prime}+2 \delta}} .
$$

By (3.15) and (4.20), we have for $T \in \mathcal{F}^{\prime}$

$$
\inf _{x \in I_{T}} M_{p_{j}} f_{j}(x) \geq C 2^{-\frac{\mu}{p_{j}^{\prime}}}
$$

which gives

$$
\bigcup_{T \in \mathcal{F}^{\prime}} I_{T} \subset\left\{x \in \mathbb{R}: M_{p_{j}} f_{j}(x) \geq C 2^{-\frac{\mu}{p_{j}^{\prime}}}\right\}
$$


Thus, we have

$$
\left|\bigcup_{T \in \mathcal{F}^{\prime}} I_{T}\right| \leq C 2^{\frac{p_{j} \mu}{p_{j}^{\prime}}}
$$

Therefore, we have

$$
\left\|\left(\mathcal{N}_{\mathcal{F}^{\prime}}^{\frac{p_{j}-\delta}{p_{j}^{\prime}+\delta}}\right)^{\sharp}\right\|_{\frac{p_{j}^{\prime}+2 \delta}{p_{j}-\delta}} \leq C 2^{\frac{p_{j} \mu}{p_{j}^{\prime}}} \lambda^{12 \varepsilon}+C 2^{\frac{p_{j} \mu}{p_{j}^{\prime}}} e^{-A} \lambda 2^{\frac{p_{j}\left(p_{j}-\delta\right) \mu}{p_{j}^{\prime}\left(p_{j}^{\prime}+2 \delta\right)}} \leq C 2^{\frac{p_{j} \mu}{p_{j}^{\prime}}} \lambda^{12 \varepsilon},
$$

since $\lambda \geq 2^{\eta \mu}$. Hence, we have

$$
\int \mathcal{N}_{\mathcal{F}^{\prime}}^{\frac{p_{j}^{\prime}+2 \delta}{p_{j}^{\prime}+\delta}}(x) d x \leq C 2^{\frac{p_{j}\left(p_{j}^{\prime}+2 \delta\right) \mu}{\left(p_{j}-\delta\right) p_{j}^{\prime}}} \lambda^{12 p_{j}^{\prime} \varepsilon}
$$

which implies

$$
\left|\left\{x \in \mathbb{R}: \mathcal{N}_{\mathcal{F}^{\prime}}(x) \geq \lambda\right\}\right| \leq C 2^{\frac{p_{j}\left(p_{j}^{\prime}+2 \delta\right) \mu}{\left(p_{j}-\delta\right) p_{j}^{\prime}}} \lambda^{12 p_{j}^{\prime} \varepsilon} \lambda^{-\frac{p_{j}^{\prime}+2 \delta}{p_{j}^{\prime}+\delta}} .
$$

Choosing $\varepsilon \ll \delta \ll \eta$, we obtain (8.2) and so we are done.

\section{An application}

We consider general bilinear singular integrals on $\mathbb{R} \times \mathbb{R}$ whose kernels are homogeneous functions in $\mathbb{R}^{2}$. These have the form

$$
T_{\Omega}\left(f_{1}, f_{2}\right)(x)=\text { p.v. } \int_{\mathbb{R}^{2}} f_{1}\left(x-t_{1}\right) f_{2}\left(x-t_{2}\right) \frac{\Omega\left(\left(t_{1}, t_{2}\right) /\left|\left(t_{1}, t_{2}\right)\right|\right)}{\left|\left(t_{1}, t_{2}\right)\right|^{2}} d t_{1} d t_{2}
$$

where $\Omega\left(t_{1}, t_{2}\right)$ is an integrable function on $\mathbf{S}^{1}$ and $x \in \mathbb{R}$. $\quad\left(\left|\left(t_{1}, t_{2}\right)\right|=\right.$ $\sqrt{t_{1}^{2}+t_{2}^{2}}$ denotes the euclidean norm of the element $\left(t_{1}, t_{2}\right) \in \mathbb{R}^{2}$.)

Operators of the type (9.1) have been systematically studied by [4] and [5] and recently by [9]. The last authors obtained bounds for $T_{\Omega}$ when $\Omega$ possess a certain amount of smoothness, such as Lipschitz continuity of order $0<$ $\varepsilon<1$ on $\mathbf{S}^{1}$. As an application of Theorem 2, here we obtain that the operator $T_{\Omega}$ is bounded from $L^{p_{1}}(\mathbb{R}) \times L^{p_{2}}(\mathbb{R}) \rightarrow L^{p}(\mathbb{R})$ when the triple $\left(p_{1}, p_{2}, p\right)$ satisfies the hypotheses of Theorem 2 and $\Omega$ is merely integrable and odd.

As in the classical linear theory [2] we apply the method of rotations to the operator $T_{\Omega}$. Using polar coordinates in $\mathbb{R}^{2}$ we can write

$$
T_{\Omega}\left(f_{1}, f_{2}\right)(x)=\int_{\mathbf{S}^{1}} \Omega\left(\theta_{1}, \theta_{2}\right)\left\{\int_{0}^{+\infty} f_{1}\left(x-t \theta_{1}\right) f_{2}\left(x-t \theta_{2}\right) \frac{d t}{t}\right\} d\left(\theta_{1}, \theta_{2}\right)
$$


Replacing $\theta$ by $-\theta$, changing variables, and using that $\Omega$ is odd we obtain

$$
T_{\Omega}\left(f_{1}, f_{2}\right)(x)=\int_{\mathbf{S}^{1}} \Omega\left(\theta_{1}, \theta_{2}\right)\left\{\int_{0}^{+\infty} f_{1}\left(x+t \theta_{1}\right) f_{2}\left(x+t \theta_{2}\right) \frac{d t}{t}\right\} d\left(\theta_{1}, \theta_{2}\right)
$$

Averaging (9.2) and (9.3) yields

$$
T_{\Omega}\left(f_{1}, f_{2}\right)(x)=\frac{1}{2} \int_{\mathbf{S}^{1}} \Omega\left(\theta_{1}, \theta_{2}\right)\left\{\int_{-\infty}^{+\infty} f_{1}\left(x-t \theta_{1}\right) f_{2}\left(x-t \theta_{2}\right) \frac{d t}{t}\right\} d\left(\theta_{1}, \theta_{2}\right)
$$

But the operator inside the curly brackets above is no other than $H_{\theta_{1}, \theta_{2}}$, which was shown to be bounded from $L^{p_{1}}(\mathbb{R}) \times L^{p_{2}}(\mathbb{R}) \rightarrow L^{p}(\mathbb{R})$ uniformly in $\theta_{1}, \theta_{2}$, when the triple $\left(p_{1}, p_{2}, p\right)$ satisfies the hypotheses of Theorem 2 . It follows that $T_{\Omega}$ is also bounded from $L^{p_{1}}(\mathbb{R}) \times L^{p_{2}}(\mathbb{R}) \rightarrow L^{p}(\mathbb{R})$ for the same range of $p$ 's when $\Omega$ is odd and integrable.

Thus the operators $H_{\theta_{1}, \theta_{2}}$ play the role of the linear directional Hilbert transforms in the theory of bilinear singular integrals. This reason justifies their name bilinear (directional) Hilbert transforms.

We end by noting that Calderón's identity

$$
\mathcal{C}_{1}(f ; A)(x)=\text { p.v. } \int_{\mathbb{R}} \frac{A(x)-A(y)}{(x-y)^{2}} f(y) d y=\int_{0}^{1} H_{1, \alpha}\left(f, A^{\prime}\right)(x) d \alpha
$$

can be thought as a special case of (9.4) when $\Omega$ is suitably chosen.

\section{References}

[1] Calderón, A.: Commutators of singular integral operators. Proc. Nat. Acad. Sci. U.S.A. 53 (1977), 1092-1099.

[2] Calderón, A.P. And Zygmund, A.: On singular integrals. Amer. J. Math. 78 (1956), 289-309.

[3] Carleson, L.: On convergence and growth of partials sumas of Fourier series. Acta Math. 116 (1966), 135-157.

[4] Colfman, R. R. And Meyer, Y.: On commutators of singular integrals and bilinear singular integrals. Trans. Amer. Math. Soc. 212 (1975), 315331.

[5] Coifman, R. R. And Meyer, Y.: Commutateurs d' intégrales singulières et opérateurs multilinéaires. Ann. Inst. Fourier (Grenoble) 28 (1978), 177202.

[6] Fefferman, C.: Pointwise convergence of Fourier series. Ann. of Math. (2) 98 (1973), 551-571.

[7] Fefferman, C. And Stein, E. M.: Some maximal inequalities. Amer. J. Math. 93 (1971), 107-115. 
[8] Grafakos, L. And Li, X.: Uniform bounds for the bilinear Hilbert transforms. I. Ann. of Math. (2) 159 (2004), 889-933.

[9] Grafakos, L. And Torres, R.: Multilinear Calderón-Zygmund theory. Adv. Math. 165 (2002), no. 1, 124-164.

[10] Hunt R. A.: On the convergence of Fourier Series. In 1968 Orthogonal Expansions and their Continuous Analogues (Proc. Conf. Edwardsville, 1967), 235-255. D. T. Haimo (ed), Southern Illinois Univ. Press, Carbondale Ill.

[11] Lacey, M. T. and Thiele, C. M.: $L^{p}$ estimates on the bilinear Hilbert transform for $2<p<\infty$. Ann. of Math. (2) 146 (1997), 693-724.

[12] Lacey, M. T. And Thiele, C. M.: On Calderón's conjecture. Ann. of Math. (2) 149 (1999), 475-496.

[13] Stein, E. M.: Harmonic analysis: real-variable methods, orthogonality, and oscillatory integrals. Princeton Mathematical Series 43. Monographs in Harmonic Analysis, III. Princeton University Press, Princeton, NJ, 1993.

[14] Thiele, C. M.: On the Bilinear Hilbert Transform. Habilitationsschrift, Universität Kiel, 1998.

[15] Thiele, C. M.: A uniform estimate. Ann. of Math. (2) 156 (2002), 519-563.

Recibido: 24 de febrero de 2005

Xiaochun Li

Department of Mathematics

University of Illinois at Urbana-Champaign

Urbana, IL 61801-2975, USA

xcli@math.uiuc.edu

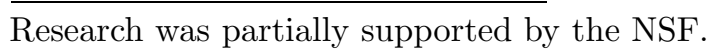

